\title{
Rapid Construction of Tetralin, Chromane, and Indane Motifs via Cyclative C-H/C-H Coupling: Four-Step Total Synthesis of ( \pm )-Russujaponol F
}

\author{
Zhe Zhuang, ${ }^{1}$ Alastair N. Herron, ${ }^{1}$ Shuang Liu, ${ }^{1}$ and Jin-Quan $\mathrm{Yu}^{1 *}$ \\ ${ }^{1}$ Department of Chemistry, The Scripps Research Institute, 10550 N. Torrey Pines Road, La \\ Jolla, CA 92037, United States
}

\section{Table of Contents}

General Information

Experimental Section

Preparation of aliphatic acids

Preparation of mono- $N$-protected $\beta$-amino acid ligand

Table S1. Oxidant investigation for the cyclative $\mathrm{C}-\mathrm{H} / \mathrm{C}-\mathrm{H}$ coupling reaction

Table S2. Base screening for the cyclative $\mathrm{C}-\mathrm{H} / \mathrm{C}-\mathrm{H}$ coupling reaction

Table S3. Ligand investigation for the cyclative $\mathrm{C}-\mathrm{H} / \mathrm{C}-\mathrm{H}$ coupling reaction

Table S4. Comparison between LiOAc and NaOAc under the standard conditions

Table S5. Conditions investigation for the cyclative $\mathrm{C}-\mathrm{H} / \mathrm{C}-\mathrm{H}$ coupling reaction of $\mathbf{1 v} \mathrm{S} 10$

Table S6. Base and Ag salt investigation for arylation

General procedure for the cyclative $\mathrm{C}-\mathrm{H} / \mathrm{C}-\mathrm{H}$ coupling reaction

Substrate scope of the cyclative $\mathrm{C}-\mathrm{H} / \mathrm{C}-\mathrm{H}$ coupling reaction

Total synthesis of $( \pm)$-russujaponol F 


\section{General Information}

$\mathrm{Pd}(\mathrm{OAc})_{2}$, $\mathrm{LiOAc}, \mathrm{Ag}_{2} \mathrm{CO}_{3}$, and sodium percarbonate $\left(\mathrm{Na}_{2} \mathrm{CO}_{3} \cdot 1.5 \mathrm{H}_{2} \mathrm{O}_{2}\right)$ were purchased from Sigma-Aldrich. $\quad \mathrm{Pd}\left(\mathrm{CH}_{3} \mathrm{CN}\right)_{4}\left(\mathrm{BF}_{4}\right)_{2}$ was purchased from Strem. 1-Fluoro-2,4,6trimethylpyridinium tetrafluoroborate was purchased from TCI. HFIP was purchased from Oakwood. Other reagents were purchased at the highest commercial quality and used without further purification, unless otherwise stated. Analytical thin layer chromatography was performed on $0.25 \mathrm{~mm}$ silica gel 60-F254. Visualization was carried out with short-wave UV light or $\mathrm{KMnO}_{4}$ and heat as developing agents. ${ }^{1} \mathrm{H}$ NMR spectra were recorded on Bruker DRX-600 instrument. Chemical shifts were quoted in parts per million ( $\mathrm{ppm}$ ) referenced to $0.00 \mathrm{ppm}$ for TMS. The following abbreviations (or combinations thereof) were used to explain multiplicities: $\mathrm{s}=$ singlet, $\mathrm{d}=$ doublet, $\mathrm{t}=$ triplet, $\mathrm{q}=$ quartet, $\mathrm{m}=$ multiplet, $\mathrm{br}=$ broad . Coupling constants, $J$, were reported in Hertz unit $(\mathrm{Hz}) .{ }^{13} \mathrm{C}$ NMR spectra were recorded on Bruker DRX-600 was fully decoupled by broad band proton decoupling. Chemical shifts were reported in ppm referenced to the center line of a triplet at $77.16 \mathrm{ppm}$ of $\mathrm{CDCl}_{3}$. Column chromatography was performed using E. Merck silica (60, particle size $0.043-0.063 \mathrm{~mm}$ ), and preparative thin layer chromatography (pTLC) was performed on Merck silica plates (60F-254). High-resolution mass spectra (HRMS) were recorded on an Agilent Mass spectrometer using ESI-TOF (electrospray ionization-time of flight). 
Preparation of aliphatic acids<smiles>CCC(CC)(CCc1ccccc1)C(=O)O</smiles>

$1 \mathrm{a}$<smiles>CCC(CC)(CCc1cccc2ccccc12)C(=O)O</smiles>

$1 e$<smiles>C=CC(CCc1ccccc1)C(=O)O</smiles>

$1 \mathrm{i}$<smiles>CC(C)(Oc1ccccc1)C(=O)O</smiles>

$1 \mathrm{~m}$<smiles>CCC(C)(Oc1ccccc1Br)C(=O)O</smiles>

$1 q$<smiles>CCC(C=O)(CC)C(=O)O</smiles>

$1 \mathrm{u}$<smiles>CCC(CC)(CCc1ccc(C)cc1)C(=O)O</smiles>

$1 b$<smiles>CCC(C=O)(CCc1ccc(F)cc1)C(=O)O</smiles>

$1 \mathrm{c}$<smiles>CC(C)(Cc1ccc(F)cc1)C(=O)O</smiles>

$1 g$<smiles>COc1ccccc1CCC(C=O)C(=O)O</smiles>

$1 \mathrm{k}$<smiles>CC(C)(Oc1cccc(Br)c1)C(=O)O</smiles>

$1 n$<smiles>[M]C(C)(Oc1ccccc1C(F)(F)F)C(=O)O</smiles>

$1 \mathrm{r}$<smiles>Cc1ccccc1CC(C)(C)C(=O)O</smiles>

1v<smiles>CC(C)(Oc1cccc2c1CCCC2)C(=O)O</smiles>

10<smiles>COc1cccc(OC[C@H](C)C(=O)O)c1</smiles>

$1 \mathrm{~s}$<smiles>CCC(C=O)(CCc1ccc(Cl)cc1)C(=O)O</smiles>

1d

$1 \mathrm{~h}$<smiles>C=CC(CCc1ccc(F)cc1)C(=O)O</smiles>

11<smiles>CC(Oc1ccccc1Br)C(C)(C)C(=O)O</smiles>

$1 p$<smiles>[R]N([R])C(C)(C)C(=O)O</smiles>

$\mathrm{R}=\mathrm{Boc}$ or $\mathrm{Ts}$ $1 \mathrm{t}$ 


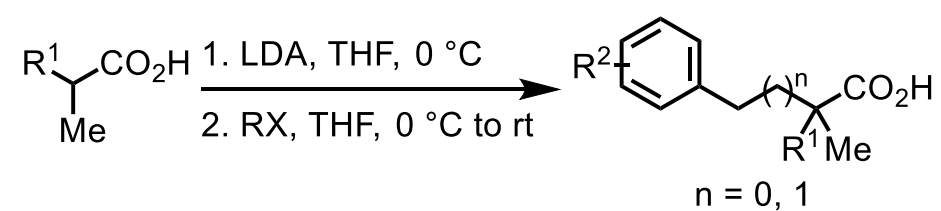

1a-h and $1 \mathrm{u}-\mathrm{v}$

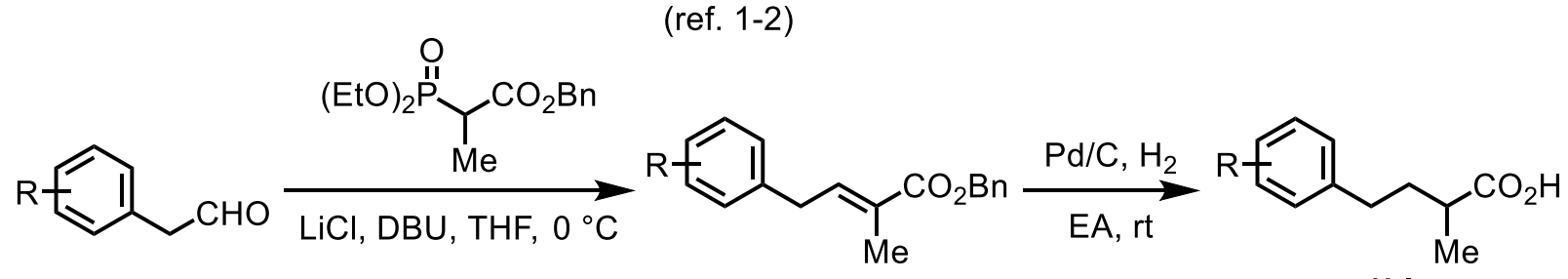

$1 \mathrm{i}-\mathrm{I}$ (ref. 3)

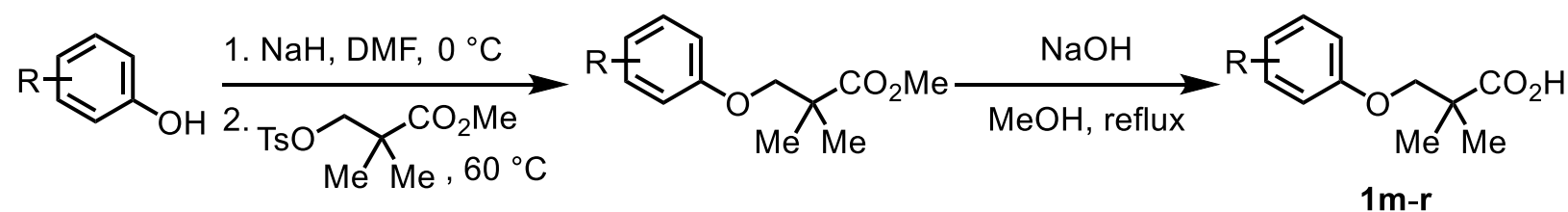<smiles>COc1cccc(O)c1</smiles>

$1 \mathrm{~s}$ (ref. 4)<smiles>COC(OC)=C(C)C(OC)O[Na]</smiles>

Aliphatic carboxylic acids were synthesized following literature procedures ${ }^{1-5}(\mathbf{1 a}-\mathbf{1 v})$ or obtained from the commercial source (1w). 
Preparation of mono- $N$-protected $\beta$-amino acid ligand

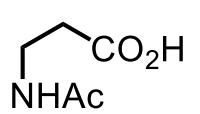

L4<smiles>NCC(Cc1ccccc1)C(=O)O</smiles>

L8<smiles>CC(C)NC(C)CC(=O)O</smiles>

L5

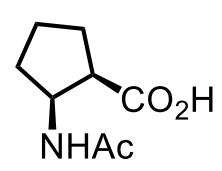

(士)-L9<smiles>CC(C)(C)NC(CCC(=O)O)Cc1ccccc1</smiles>

L6

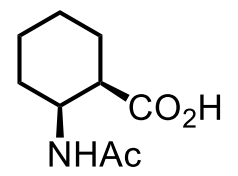

(士)-L10
$\sum_{\mathrm{NHAC}}^{\mathrm{Me}} \mathrm{CO}_{2} \mathrm{H}$

L7

L4-L10 were commercially available $\left(\mathbf{L 4 )}\right.$ or synthesized following literature procedures ${ }^{6-9}$ (L5-L10). 
Table S1. Oxidant investigation for the cyclative $\mathrm{C}-\mathrm{H} / \mathrm{C}-\mathrm{H}$ coupling reaction ${ }^{a, b}$<smiles>CCC(CC)(CCc1ccccc1)C(=O)O</smiles>

$1 \mathrm{a}$

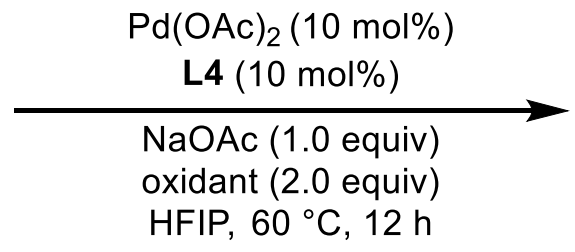

HFIP, $60^{\circ} \mathrm{C}, 12 \mathrm{~h}$

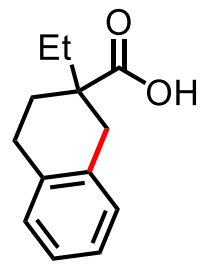

$2 a$

\begin{tabular}{ccc:ccc}
\hline entry & oxidant & yield $(\%)$ & entry & oxidant & yield (\%) \\
\hline 1 & w/o & 0 & 10 & CMHP & 22 \\
2 & AcOO ${ }^{t} \mathrm{Bu}$ & 38 & 11 & TBHP (70\% in water) & 50 \\
3 & $\mathrm{BzOO}{ }^{t} \mathrm{Bu}$ & 0 & 12 & TBHP (ca. 5.5 M in decane) & 53 \\
4 & $\mathrm{BzOOBz}$ & 0 & 13 & $\mathrm{~K}_{2} \mathrm{~S}_{2} \mathrm{O}_{8}$ & 5 \\
5 & Lauroyl peroxide & 0 & 14 & $\mathrm{Na}_{2} \mathrm{~S}_{2} \mathrm{O}_{8}$ & 0 \\
6 & ${ }^{t} \mathrm{BuOO}{ }^{t} \mathrm{Bu}$ & 0 & 15 & Oxone & 0 \\
7 & $\mathrm{H}_{2} \mathrm{O}_{2}$ in water & 0 & 16 & Selectfluor & 32 \\
8 & $\mathrm{UHP}$ & 0 & 17 & NFSI & 0 \\
9 & $\mathrm{Na}_{2} \mathrm{CO}_{3} \cdot 1.5 \mathrm{H}_{2} \mathrm{O}_{2}$ & 56 & 18 & FTMP & 0 \\
\hline
\end{tabular}

${ }^{a}$ Conditions: 1a ( $\left.0.1 \mathrm{mmol}\right), \mathrm{Pd}(\mathrm{OAc})_{2}(10 \mathrm{~mol} \%), \mathbf{L 4}(10 \mathrm{~mol} \%), \mathrm{NaOAc}$ (1.0 equiv), $\mathrm{Na}_{2} \mathrm{CO}_{3} \cdot 1.5 \mathrm{H}_{2} \mathrm{O}_{2}$ (2.0 equiv), HFIP (1.0 mL), $60{ }^{\circ} \mathrm{C}, 12 \mathrm{~h} .{ }^{b}$ The yields were determined by ${ }^{1} \mathrm{H}$ NMR analysis of the crude product using $\mathrm{CH}_{2} \mathrm{Br}_{2}$ as the internal standard. 
Table S2. Base screening for the cyclative $\mathrm{C}-\mathrm{H} / \mathrm{C}-\mathrm{H}$ coupling reaction ${ }^{a, b}$<smiles>CCC(C=O)(CCc1ccccc1)C(=O)O</smiles>

$1 \mathrm{a}$

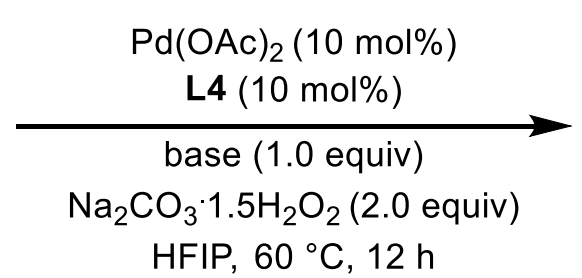

HFIP, $60^{\circ} \mathrm{C}, 12 \mathrm{~h}$

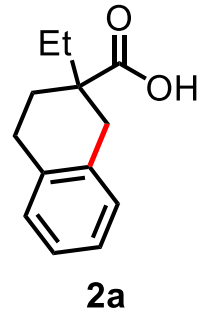

2a

\begin{tabular}{ccc:cccc}
\hline entry & base & yield $(\%)$ & entry & base & yield (\%) \\
\hline 1 & w/o & 50 & 6 & $\mathrm{Na}_{3} \mathrm{PO}_{4}$ & 49 \\
2 & $\mathrm{NaHCO}_{3}$ & 45 & 7 & $\mathrm{NaOAc}$ & 56 \\
3 & $\mathrm{Na}_{2} \mathrm{CO}_{3}$ & 44 & 8 & $\mathrm{LiOAc}$ & 57 \\
4 & $\mathrm{NaH}_{2} \mathrm{PO}_{4}$ & 36 & 9 & $\mathrm{KOAc}$ & 50 \\
5 & $\mathrm{Na}_{2} \mathrm{HPO}_{4}$ & 45 & 10 & $\mathrm{CsOAc}$ & 41 \\
\hline
\end{tabular}

${ }^{a}$ Conditions: 1a $(0.1 \mathrm{mmol}), \mathrm{Pd}(\mathrm{OAc})_{2}(10 \mathrm{~mol} \%), \mathbf{L 4}(10 \mathrm{~mol} \%)$, base (1.0 equiv), $\mathrm{Na}_{2} \mathrm{CO}_{3} \cdot 1.5 \mathrm{H}_{2} \mathrm{O}_{2}(2.0$ equiv), HFIP $(1.0 \mathrm{~mL}), 60{ }^{\circ} \mathrm{C}, 12 \mathrm{~h} .{ }^{b}$ The yields were determined by ${ }^{1} \mathrm{H}$ NMR analysis of the crude product using $\mathrm{CH}_{2} \mathrm{Br}_{2}$ as the internal standard. The conversions were determined by ${ }^{1} \mathrm{H}$ NMR analysis of the remaining $1 \mathbf{a}$. 
Table S3. Ligand investigation for the cyclative $\mathrm{C}-\mathrm{H} / \mathrm{C}-\mathrm{H}$ coupling reaction ${ }^{a, b}$<smiles>CCC(C=O)(CCc1ccccc1)C(=O)O</smiles>

$1 \mathrm{a}$

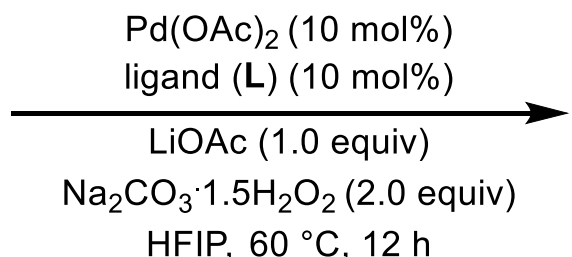

HFIP, $60^{\circ} \mathrm{C}, 12 \mathrm{~h}$

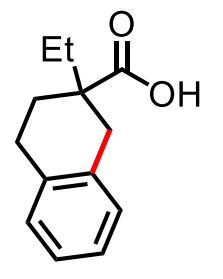

2a

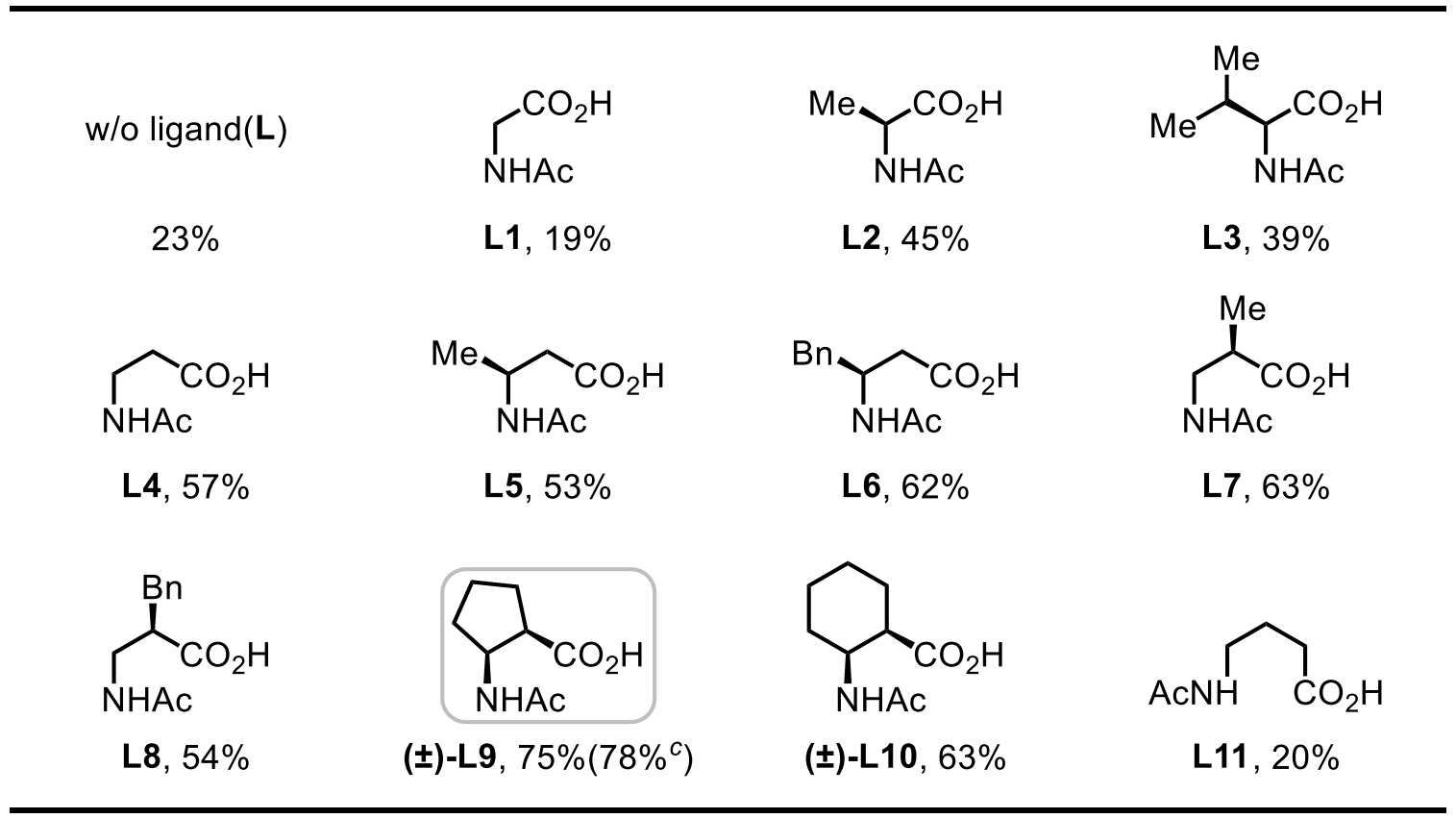

${ }^{a}$ Conditions: 1a $(0.1 \mathrm{mmol}), \mathrm{Pd}(\mathrm{OAc})_{2}(10 \mathrm{~mol} \%)$, ligand $(\mathbf{L})(10 \mathrm{~mol} \%)$, LiOAc (1.0 equiv), $\mathrm{Na}_{2} \mathrm{CO}_{3} \cdot 1.5 \mathrm{H}_{2} \mathrm{O}_{2}(2.0$ equiv), HFIP (1.0 mL), $60{ }^{\circ} \mathrm{C}, 12 \mathrm{~h} .{ }^{b}$ The yields were determined by ${ }^{1} \mathrm{H}$ NMR analysis of the crude product using $\mathrm{CH}_{2} \mathrm{Br}_{2}$ as the internal standard. Isolated yield. 
Table S4. Comparison between LiOAc and NaOAc under the standard conditions ${ }^{a, b}$<smiles>[H][R]([H])(CC)C(=O)O</smiles>

$\mathrm{X}=\mathrm{C}, \mathrm{O}$

$\mathrm{n}=0,1$

1

\begin{tabular}{cccc}
\hline entry & 1 & yield using LiOAc $(\%)$ & yield using NaOAc $(\%)$ \\
\hline 1 & 1a & 78 & 61 \\
2 & 1d & 58 & 45 \\
3 & 1i & 63 & 45 \\
4 & 1n & 80 & 77 \\
5 & 1u & 53 & 44 \\
\hline
\end{tabular}

${ }^{a}$ Conditions: 1 (0.1 mmol), $\mathrm{Pd}(\mathrm{OAc})_{2}(10 \mathrm{~mol} \%),( \pm)-\mathbf{L 9}(10 \mathrm{~mol} \%)$, base (1.0 equiv), $\mathrm{Na}_{2} \mathrm{CO}_{3} \cdot 1.5 \mathrm{H}_{2} \mathrm{O}_{2}(2.0$ equiv), HFIP (1.0 mL), $60{ }^{\circ} \mathrm{C}, 12 \mathrm{~h} .{ }^{b}$ Isolated yields. 
Table S5. Conditions investigation for the cyclative $\mathrm{C}-\mathrm{H} / \mathrm{C}-\mathrm{H}$ coupling reaction ${ }^{a, b}$<smiles>Cc1ccccc1CC(C)(C)C(=O)O</smiles>

$1 v$

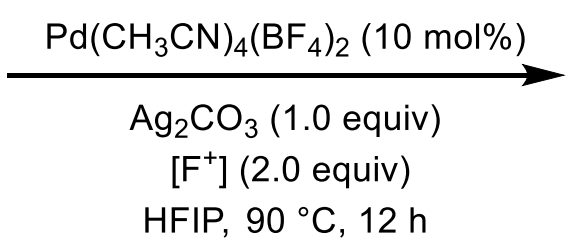

HFIP, $90^{\circ} \mathrm{C}, 12 \mathrm{~h}$<smiles>Cc1cccc2c1CC(C)(C(=O)O)C2</smiles>

2v

\begin{tabular}{|c|c|c|}
\hline entry & variation from standard conditions $B$ & yield $(\%)$ \\
\hline 1 & none & $55\left(61^{c}\right)$ \\
\hline 2 & w/o $\mathrm{Ag}_{2} \mathrm{CO}_{3}$ & 0 \\
\hline 3 & $\mathrm{Na}_{2} \mathrm{CO}_{3}$ instead of $\mathrm{Ag}_{2} \mathrm{CO}_{3}$ & 0 \\
\hline 4 & LiOAc instead of $\mathrm{Ag}_{2} \mathrm{CO}_{3}$ & 0 \\
\hline 5 & $\mathrm{Pd}(\mathrm{OAc})_{2}$ instead of $\mathrm{Pd}\left(\mathrm{CH}_{3} \mathrm{CN}\right)_{4}\left(\mathrm{BF}_{4}\right)_{2}$ & 23 \\
\hline 6 & w/ (士)-L9 (10 mol\%) & 34 \\
\hline 7 & standard conditions A & 23 \\
\hline
\end{tabular}

${ }^{a}$ Conditions: $1 v$ (0.1 mmol), $\mathrm{Pd}\left(\mathrm{CH}_{3} \mathrm{CN}\right)_{4}\left(\mathrm{BF}_{4}\right)_{2}$ (10 mol\%), $\mathrm{Ag}_{2} \mathrm{CO}_{3}$ (1.0 equiv), $\left[\mathrm{F}^{+}\right]=1$-fluoro-2,4,6trimethylpyridinium tetrafluoroborate (2.0 equiv), HFIP $(1.0 \mathrm{~mL}), 90{ }^{\circ} \mathrm{C}, 12 \mathrm{~h} .{ }^{b}$ The yields were determined by ${ }^{1} \mathrm{H}$ NMR analysis of the crude product using $\mathrm{CH}_{2} \mathrm{Br}_{2}$ as the internal standard. ${ }^{c}$ Isolated yield. 
Table S6. Base and Ag salt investigation for arylation ${ }^{a, b}$

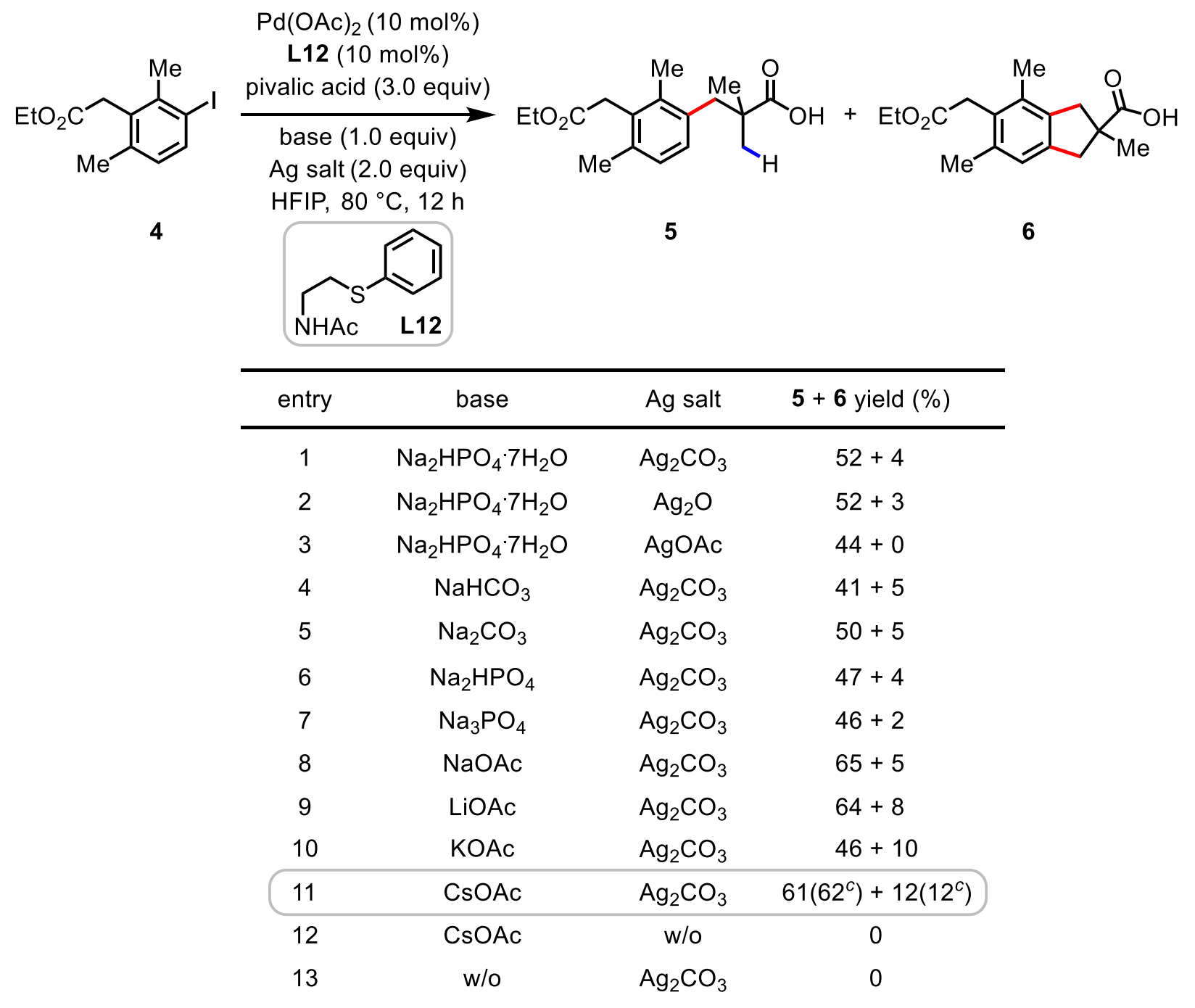

${ }^{a}$ Conditions: 4 (0.1 mmol), pivalic acid (3.0 equiv), $\mathrm{Pd}(\mathrm{OAc})_{2}(10 \mathrm{~mol} \%), \mathbf{L 1 2}(10 \mathrm{~mol} \%)$, base (1.0 equiv), $\mathrm{Ag}$ salt (2.0 equiv), HFIP (1.0 mL), $80{ }^{\circ} \mathrm{C}, 12 \mathrm{~h} .{ }^{b}$ The yields were determined by ${ }^{1} \mathrm{H}$ NMR analysis of the crude product using $\mathrm{CH}_{2} \mathrm{Br}_{2}$ as the internal standard. ${ }^{c}$ Isolated yields. 


\section{General procedure for the cyclative $\mathrm{C}-\mathrm{H} / \mathrm{C}-\mathrm{H}$ coupling reaction}
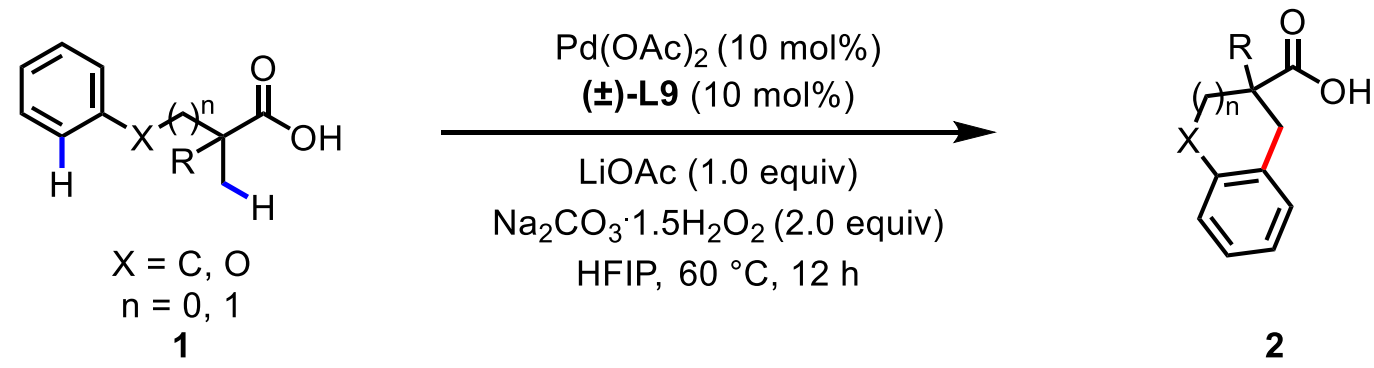

General Procedure A: In the culture tube, $\mathrm{Pd}(\mathrm{OAc})_{2}(10 \mathrm{~mol} \%, 2.2 \mathrm{mg})$, ligand ( \pm )-L9 (10 mol\%, $1.7 \mathrm{mg}$ ), $\mathrm{LiOAc}$ (1.0 equiv, $6.6 \mathrm{mg}), \mathrm{Na}_{2} \mathrm{CO}_{3} \cdot 1.5 \mathrm{H}_{2} \mathrm{O}_{2}(2.0$ equiv, $31.4 \mathrm{mg})$, and 1 (0.1 mmol) in order were weighed in air and placed with a magnetic stir bar. Then HFIP (1.0 mL) was added. The reaction mixture was stirred at $\mathrm{rt}$ for $3 \mathrm{~min}$, and then heated to $60{ }^{\circ} \mathrm{C}$ for $12 \mathrm{~h}(600 \mathrm{rpm})$. After being allowed to cool to room temperature, the mixture was treated with $\mathrm{HCO}_{2} \mathrm{H}(0.1 \mathrm{~mL})$ and concentrated in vacuo. The crude mixture was purified by pTLC (hexane/EA with $1 \% \mathrm{AcOH}$ ) to afford the product 2 .

General Procedure B: In the culture tube, $\mathrm{Pd}\left(\mathrm{CH}_{3} \mathrm{CN}\right)_{4}\left(\mathrm{BF}_{4}\right)_{2}(10 \mathrm{~mol} \%, 4.4 \mathrm{mg}), \mathrm{Ag}_{2} \mathrm{CO}_{3}(1.0$ equiv, $27.4 \mathrm{mg}$ ), 1-fluoro-2,4,6-trimethylpyridinium tetrafluoroborate (2.0 equiv, $45.4 \mathrm{mg}$ ), and 1 $(0.1 \mathrm{mmol})$ in order were weighed in air and placed with a magnetic stir bar. Then HFIP (1.0 mL) was added. The reaction mixture was stirred at $\mathrm{rt}$ for $3 \mathrm{~min}$, and then heated to $90{ }^{\circ} \mathrm{C}$ for $12 \mathrm{~h}(600$ $\mathrm{rpm})$. After being allowed to cool to room temperature, the mixture was treated with $\mathrm{HCO}_{2} \mathrm{H}(0.1$ $\mathrm{mL}$ ), diluted with DCM, filtered through a Celite plug, and concentrated in vacuo. The crude mixture was purified by pTLC (hexane/EA with $1 \% \mathrm{AcOH}$ ) to afford the product 2. 
Substrate scope of the cyclative $\mathrm{C}-\mathrm{H} / \mathrm{C}-\mathrm{H}$ coupling reaction<smiles>CCC1(C(=O)O)CCc2ccccc2C1</smiles>

\section{2-Ethyl-1,2,3,4-tetrahydronaphthalene-2-carboxylic acid (2a)}

Following General Procedure A on $0.1 \mathrm{mmol}$ scale. Purification by pTLC afforded the title compound (colorless oil, $16.0 \mathrm{mg}, 78 \%$ yield).

Following General Procedure A on $2.0 \mathrm{mmol}$ scale. Purification by column chromatography afforded the title compound ( $282.0 \mathrm{mg}, 69 \%$ yield).

${ }^{1} \mathrm{H}$ NMR (600 MHz, $\left.\mathrm{CDCl}_{3}\right) \delta 7.14-7.03(\mathrm{~m}, 4 \mathrm{H}), 3.22$ (d, J=16.5 Hz, 1H), $2.92-2.83(\mathrm{~m}, 1 \mathrm{H})$, $2.83-2.75(\mathrm{~m}, 1 \mathrm{H}), 2.67(\mathrm{~d}, J=16.5 \mathrm{~Hz}, 1 \mathrm{H}), 2.20-2.12(\mathrm{~m}, 1 \mathrm{H}), 1.85-1.77(\mathrm{~m}, 1 \mathrm{H}), 1.79-$ $1.69(\mathrm{~m}, 1 \mathrm{H}), 1.70-1.61(\mathrm{~m}, 1 \mathrm{H}), 0.94(\mathrm{t}, J=7.5 \mathrm{~Hz}, 3 \mathrm{H})$.

${ }^{13} \mathrm{C} \mathrm{NMR}\left(150 \mathrm{MHz}, \mathrm{CDCl}_{3}\right) \delta 182.5,135.5,134.9,129.3,128.8,126.0,125.9,46.0,36.6,31.1$, $30.1,26.3,8.9$.

HRMS (ESI-TOF) Calcd for $\mathrm{C}_{13} \mathrm{H}_{15} \mathrm{O}_{2}^{-}[\mathrm{M}-\mathrm{H}]^{-}: 203.1078$; found: 203.1072 .

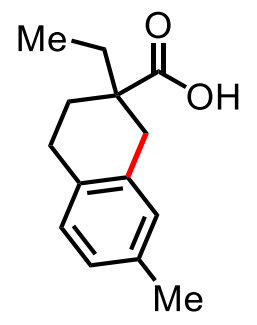

2-Ethyl-7-methyl-1,2,3,4-tetrahydronaphthalene-2-carboxylic acid (2b)

Following General Procedure A on $0.1 \mathrm{mmol}$ scale. Purification by pTLC afforded the title compound (colorless oil, $16.5 \mathrm{mg}, 76 \%$ yield).

${ }^{1} \mathrm{H}$ NMR $\left(600 \mathrm{MHz}, \mathrm{CDCl}_{3}\right) \delta 7.00-6.93(\mathrm{~m}, 1 \mathrm{H}), 6.93-6.85(\mathrm{~m}, 2 \mathrm{H}), 3.17(\mathrm{~d}, J=16.4 \mathrm{~Hz}, 1 \mathrm{H})$, $2.87-2.78(\mathrm{~m}, 1 \mathrm{H}), 2.78-2.70(\mathrm{~m}, 1 \mathrm{H}), 2.63(\mathrm{~d}, J=16.4 \mathrm{~Hz}, 1 \mathrm{H}), 2.28(\mathrm{~s}, 3 \mathrm{H}), 2.18-2.08(\mathrm{~m}$, 1H), $1.84-1.75(\mathrm{~m}, 1 \mathrm{H}), 1.77-1.68(\mathrm{~m}, 1 \mathrm{H}), 1.69-1.59(\mathrm{~m}, 1 \mathrm{H}), 0.93(\mathrm{t}, J=7.4 \mathrm{~Hz}, 3 \mathrm{H})$.

${ }^{13} \mathrm{C} \mathrm{NMR}\left(150 \mathrm{MHz}, \mathrm{CDCl}_{3}\right.$ ) (major and minor rotamers) $\delta 182.8,135.6,135.6,135.5,134.8$, 132.6, 132.0, 130.1, 129.6, 129.4, 128.9, 127.1, 127.0, 46.3, 46.2 , 36.8, 36.5, 31.3, 31.3, 30.5, 30.3, $26.4,26.1,21.3,9.1$.

HRMS (ESI-TOF) Calcd for $\mathrm{C}_{14} \mathrm{H}_{17} \mathrm{O}_{2}^{-}[\mathrm{M}-\mathrm{H}]^{-}: 217.1234$; found: 217.1232. 


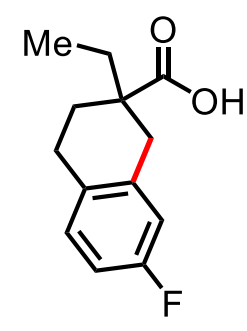

\section{2-Ethyl-7-fluoro-1,2,3,4-tetrahydronaphthalene-2-carboxylic acid (2c)}

Following General Procedure A on $0.1 \mathrm{mmol}$ scale. Purification by pTLC afforded the title compound (colorless oil, $13.0 \mathrm{mg}, 59 \%$ yield).

${ }^{1} \mathrm{H}$ NMR $\left(600 \mathrm{MHz}, \mathrm{CDCl}_{3}\right) \delta 7.06-6.97(\mathrm{~m}, 1 \mathrm{H}), 6.84-6.73(\mathrm{~m}, 2 \mathrm{H}), 3.24-3.12(\mathrm{~m}, 1 \mathrm{H}), 2.90$ $-2.71(\mathrm{~m}, 2 \mathrm{H}), 2.68-2.58(\mathrm{~m}, 1 \mathrm{H}), 2.20-2.11(\mathrm{~m}, 1 \mathrm{H}), 1.83-1.68(\mathrm{~m}, 2 \mathrm{H}), 1.68-1.60(\mathrm{~m}$, $1 \mathrm{H}), 0.98-0.90(\mathrm{~m}, 3 \mathrm{H})$.

${ }^{13} \mathrm{C} \mathrm{NMR}\left(150 \mathrm{MHz}, \mathrm{CDCl}_{3}\right)$ (major rotamer) $\delta 182.2,161.2(\mathrm{~d}, J=243.4 \mathrm{~Hz}), 136.9(\mathrm{~d}, J=7.2$ $\mathrm{Hz}), 130.9$ (d, $J=2.8 \mathrm{~Hz}), 130.1(\mathrm{~d}, J=8.2 \mathrm{~Hz}), 115.0$ (d, $J=20.4 \mathrm{~Hz}), 113.1$ (d, $J=21.3 \mathrm{~Hz})$, 45.8, 36.6, 31.3, 30.3, 25.7, 8.9.

${ }^{13} \mathrm{C} \mathrm{NMR}\left(150 \mathrm{MHz}, \mathrm{CDCl}_{3}\right)$ (minor rotamer) $\delta 182.3,161.2(\mathrm{~d}, J=243.4 \mathrm{~Hz}), 137.4(\mathrm{~d}, J=7.2$ $\mathrm{Hz}), 130.5$ (d, $J=7.8 \mathrm{~Hz}), 130.4(\mathrm{~d}, J=2.9 \mathrm{~Hz}), 115.4$ (d, $J=20.8 \mathrm{~Hz}), 115.2$ (d, $J=21.0 \mathrm{~Hz})$, 46.1, 36.0, 31.2, 29.8, 26.5, 8.9.

HRMS (ESI-TOF) Calcd for $\mathrm{C}_{13} \mathrm{H}_{14} \mathrm{FO}_{2}^{-}[\mathrm{M}-\mathrm{H}]^{-}: 221.0983$; found: 221.0990.<smiles>CCC1(C(=O)O)CCc2ccc(Cl)cc2C1</smiles>

\section{7-Chloro-2-ethyl-1,2,3,4-tetrahydronaphthalene-2-carboxylic acid (2d)}

Following General Procedure A on $0.1 \mathrm{mmol}$ scale. Purification by pTLC afforded the title compound (colorless oil, $13.8 \mathrm{mg}, 58 \%$ yield).

${ }^{1} \mathrm{H}$ NMR $\left(600 \mathrm{MHz}, \mathrm{CDCl}_{3}\right) \delta 7.11-7.03(\mathrm{~m}, 2 \mathrm{H}), 7.03-6.96(\mathrm{~m}, 1 \mathrm{H}), 3.25-3.13(\mathrm{~m}, 1 \mathrm{H}), 2.91$ $-2.71(\mathrm{~m}, 2 \mathrm{H}), 2.71-2.58(\mathrm{~m}, 1 \mathrm{H}), 2.21-2.12(\mathrm{~m}, 1 \mathrm{H}), 1.83-1.70(\mathrm{~m}, 2 \mathrm{H}), 1.69-1.60(\mathrm{~m}$, 1H), $0.94(\mathrm{t}, J=7.4 \mathrm{~Hz}, 3 \mathrm{H})$.

${ }^{13} \mathrm{C} \mathrm{NMR}\left(150 \mathrm{MHz}, \mathrm{CDCl}_{3}\right.$ ) (major and minor rotamers) $\delta 182.0,182.0,137.3,136.8,133.9$, 133.4, 131.4, 131.4, 130.6, 130.1, 129.0, 128.6, 126.1, 126.1, 46.0, 45.8, 36.4, 36.1, 31.3, 31.3, $30.1,29.9,26.3,25.8,8.9$. 
HRMS (ESI-TOF) Calcd for $\mathrm{C}_{13} \mathrm{H}_{14} \mathrm{ClO}_{2}{ }^{-}$[M-H]": 237.0688; found: 237.0684.

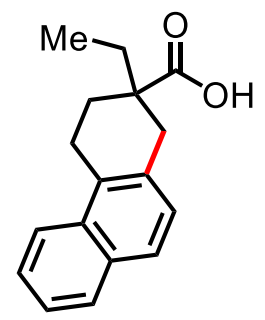

\section{2-Ethyl-1,2,3,4-tetrahydrophenanthrene-2-carboxylic acid (2e)}

Following General Procedure A on $0.1 \mathrm{mmol}$ scale. Purification by pTLC afforded the title compound (colorless oil, $11.5 \mathrm{mg}$, 45\% yield, ortho/peri $=10 / 1$ ).

${ }^{1} \mathrm{H} \mathrm{NMR}\left(600 \mathrm{MHz}, \mathrm{CDCl}_{3}\right)$ (ortho-product) $\delta 7.92(\mathrm{~d}, J=8.4 \mathrm{~Hz}, 1 \mathrm{H}), 7.78(\mathrm{~d}, J=8.0 \mathrm{~Hz}, 1 \mathrm{H})$, $7.62(\mathrm{~d}, J=8.4 \mathrm{~Hz}, 1 \mathrm{H}), 7.49$ (t, $J=7.6 \mathrm{~Hz}, 1 \mathrm{H}), 7.42(\mathrm{t}, J=7.4 \mathrm{~Hz}, 1 \mathrm{H}), 7.20(\mathrm{~d}, J=8.4 \mathrm{~Hz}, 1 \mathrm{H})$, $3.36(\mathrm{~d}, J=16.6 \mathrm{~Hz}, 1 \mathrm{H}), 3.25-3.13(\mathrm{~m}, 2 \mathrm{H}), 2.84(\mathrm{~d}, J=16.6 \mathrm{~Hz}, 1 \mathrm{H}), 2.39-2.29(\mathrm{~m}, 1 \mathrm{H})$, $2.01-1.92(\mathrm{~m}, 1 \mathrm{H}), 1.83-1.75(\mathrm{~m}, 1 \mathrm{H}), 1.76-1.67(\mathrm{~m}, 1 \mathrm{H}), 0.98(\mathrm{t}, J=7.5 \mathrm{~Hz}, 3 \mathrm{H})$.

${ }^{13} \mathrm{C}$ NMR (150 MHz, $\mathrm{CDCl}_{3}$ ) (ortho-product) $\delta 182.5,132.3,132.2,132.1,130.1,128.6,128.2$, $126.3,126.1,125.0,123.0,45.7,37.5,30.9,29.8,23.2,9.0$.

HRMS (ESI-TOF) Calcd for $\mathrm{C}_{17} \mathrm{H}_{17} \mathrm{O}_{2}^{-}$[M-H]": 253.1234; found: 253.1230 .

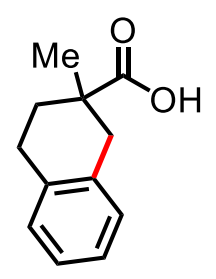

\section{2-Methyl-1,2,3,4-tetrahydronaphthalene-2-carboxylic acid (2f)}

Following General Procedure A on $0.1 \mathrm{mmol}$ scale. Purification by pTLC afforded the title compound (colorless oil, $12.5 \mathrm{mg}, 66 \%$ yield).

${ }^{1} \mathrm{H}$ NMR (600 MHz, $\left.\mathrm{CDCl}_{3}\right) \delta 7.17-7.02(\mathrm{~m}, 4 \mathrm{H}), 3.24(\mathrm{~d}, J=16.4 \mathrm{~Hz}, 1 \mathrm{H}), 2.95-2.86(\mathrm{~m}, 1 \mathrm{H})$, $2.87-2.78(\mathrm{~m}, 1 \mathrm{H}), 2.67(\mathrm{~d}, J=16.4 \mathrm{~Hz}, 1 \mathrm{H}), 2.21-2.13(\mathrm{~m}, 1 \mathrm{H}), 1.85-1.75(\mathrm{~m}, 1 \mathrm{H}), 1.32(\mathrm{~s}$, $3 \mathrm{H})$.

${ }^{13} \mathrm{C}$ NMR $\left(150 \mathrm{MHz}, \mathrm{CDCl}_{3}\right) \delta 182.7,135.1,134.7,129.4,128.9,126.0,126.0,41.6,38.5,31.8$, 26.2, 24.4.

HRMS (ESI-TOF) Calcd for $\mathrm{C}_{12} \mathrm{H}_{13} \mathrm{O}_{2}^{-}[\mathrm{M}-\mathrm{H}]^{-}:$189.0921; found: 189.0919 . 


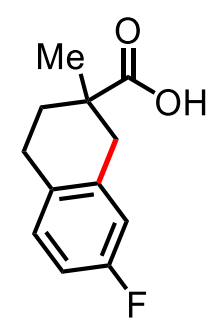

7-Fluoro-2-methyl-1,2,3,4-tetrahydronaphthalene-2-carboxylic acid (2g)

Following General Procedure A on $0.1 \mathrm{mmol}$ scale. Purification by pTLC afforded the title compound (colorless oil, $11.0 \mathrm{mg}, 53 \%$ yield).

${ }^{1} \mathrm{H}$ NMR $\left(600 \mathrm{MHz}, \mathrm{CDCl}_{3}\right) \delta 7.06$ - $6.99(\mathrm{~m}, 1 \mathrm{H}), 6.84-6.74(\mathrm{~m}, 2 \mathrm{H}), 3.26-3.14(\mathrm{~m}, 1 \mathrm{H}), 2.93$ $-2.74(\mathrm{~m}, 2 \mathrm{H}), 2.67-2.57(\mathrm{~m}, 1 \mathrm{H}), 2.22-2.12(\mathrm{~m}, 1 \mathrm{H}), 1.81-1.72(\mathrm{~m}, 1 \mathrm{H}), 1.31(\mathrm{~s}, 3 \mathrm{H})$.

${ }^{13} \mathrm{C} \mathrm{NMR}\left(150 \mathrm{MHz}, \mathrm{CDCl}_{3}\right)$ (major rotamer) $\delta 183.1,161.2(\mathrm{~d}, J=243.6 \mathrm{~Hz}), 136.7(\mathrm{~d}, J=7.3$ $\mathrm{Hz}), 130.5$ (d, $J=1.8 \mathrm{~Hz}), 130.2(\mathrm{~d}, J=7.8 \mathrm{~Hz}), 115.4$ (d, $J=20.8 \mathrm{~Hz}), 113.2(\mathrm{~d}, J=21.1 \mathrm{~Hz})$, 41.5, 38.5, 31.9, 25.6, 24.5.

${ }^{13} \mathrm{C} \mathrm{NMR}\left(150 \mathrm{MHz}, \mathrm{CDCl}_{3}\right)$ (minor rotamer) $\delta 183.2,161.2(\mathrm{~d}, J=243.6 \mathrm{~Hz}), 137.0(\mathrm{~d}, J=7.2$ $\mathrm{Hz}), 130.6(\mathrm{~d}, J=6.2 \mathrm{~Hz}), 130.2(\mathrm{~d}, J=3.1 \mathrm{~Hz}), 115.0(\mathrm{~d}, J=20.5 \mathrm{~Hz}), 113.1(\mathrm{~d}, J=21.3 \mathrm{~Hz})$, 41.7, 37.8, 31.5, 26.5, 24.5.

HRMS (ESI-TOF) Calcd for $\mathrm{C}_{12} \mathrm{H}_{12} \mathrm{FO}_{2}^{-}[\mathrm{M}-\mathrm{H}]^{-}: 207.0827$; found: 207.0825 .<smiles>CCCCC1(C(=O)O)CCc2ccccc2C1</smiles>

2-Butyl-1,2,3,4-tetrahydronaphthalene-2-carboxylic acid (2h)

Following General Procedure A on $0.1 \mathrm{mmol}$ scale. Purification by pTLC afforded the title compound (colorless oil, $16.5 \mathrm{mg}, 71 \%$ yield).

${ }^{1} \mathrm{H}$ NMR (600 MHz, $\left.\mathrm{CDCl}_{3}\right) \delta 7.13-7.03(\mathrm{~m}, 4 \mathrm{H}), 3.22$ (d, J=16.4 Hz, 1H), $2.91-2.82(\mathrm{~m}, 1 \mathrm{H})$, $2.82-2.74(\mathrm{~m}, 1 \mathrm{H}), 2.69(\mathrm{~d}, J=16.4 \mathrm{~Hz}, 1 \mathrm{H}), 2.20-2.10(\mathrm{~m}, 1 \mathrm{H}), 1.87-1.77(\mathrm{~m}, 1 \mathrm{H}), 1.73-$ $1.63(\mathrm{~m}, 1 \mathrm{H}), 1.63-1.55(\mathrm{~m}, 1 \mathrm{H}), 1.35-1.23(\mathrm{~m}, 4 \mathrm{H}), 0.89(\mathrm{t}, J=6.8 \mathrm{~Hz}, 3 \mathrm{H})$.

${ }^{13} \mathrm{C} \mathrm{NMR}\left(150 \mathrm{MHz}, \mathrm{CDCl}_{3}\right) \delta 181.4,135.3,134.7,129.1,128.6,125.7,125.7,45.3,37.9,37.0$, $30.2,26.5,26.1,23.0,13.9$.

HRMS (ESI-TOF) Calcd for $\mathrm{C}_{15} \mathrm{H}_{19} \mathrm{O}_{2}^{-}[\mathrm{M}-\mathrm{H}]^{-}: 231.1391$; found: 231.1390. 
<smiles>O=C(O)C1CCc2ccccc2C1</smiles>

\section{1,2,3,4-Tetrahydronaphthalene-2-carboxylic acid (2i)}

Following General Procedure A on $0.1 \mathrm{mmol}$ scale. Purification by pTLC afforded the title compound (colorless oil, $11.0 \mathrm{mg}, 63 \%$ yield).

${ }^{1} \mathrm{H}$ NMR $\left(600 \mathrm{MHz}, \mathrm{CDCl}_{3}\right) \delta 7.18-7.05(\mathrm{~m}, 4 \mathrm{H}), 3.10-2.98(\mathrm{~m}, 2 \mathrm{H}), 2.95-2.84(\mathrm{~m}, 2 \mathrm{H}), 2.84$ -2.77 (m, 1H), $2.29-2.22(\mathrm{~m}, 1 \mathrm{H}), 1.95-1.85(\mathrm{~m}, 1 \mathrm{H})$.

${ }^{13} \mathrm{C}$ NMR $\left(150 \mathrm{MHz}, \mathrm{CDCl}_{3}\right) \delta 181.7,135.7,134.7,129.2,129.0,126.2,126.0,39.9,31.5,28.5$, 25.8 .

HRMS (ESI-TOF) Calcd for $\mathrm{C}_{11} \mathrm{H}_{11} \mathrm{O}_{2}^{-}[\mathrm{M}-\mathrm{H}]^{-}: 175.0765$; found: 175.0757 .

The NMR data matches the reported data ${ }^{11}$.<smiles>COc1ccc2c(c1)CCC(C(=O)O)C2</smiles>

\section{6-Methoxy-1,2,3,4-tetrahydronaphthalene-2-carboxylic acid (2j)}

Following General Procedure A on $0.1 \mathrm{mmol}$ scale. Purification by pTLC afforded the title compound (colorless oil, $10.2 \mathrm{mg}, 50 \%$ yield).

${ }^{1} \mathrm{H} \mathrm{NMR}\left(600 \mathrm{MHz}, \mathrm{CDCl}_{3}\right) \delta 7.02(\mathrm{~d}, J=8.4 \mathrm{~Hz}, 1 \mathrm{H}), 6.71(\mathrm{dd}, J=8.4,2.4 \mathrm{~Hz}, 1 \mathrm{H}), 6.63(\mathrm{~s}$, $1 \mathrm{H}), 3.77(\mathrm{~s}, 3 \mathrm{H}), 3.04-2.91(\mathrm{~m}, 2 \mathrm{H}), 2.91-2.81(\mathrm{~m}, 2 \mathrm{H}), 2.81-2.73(\mathrm{~m}, 1 \mathrm{H}), 2.27-2.18(\mathrm{~m}$, $1 \mathrm{H}), 1.93-1.82(\mathrm{~m}, 1 \mathrm{H})$.

${ }^{13} \mathrm{C} \mathrm{NMR}\left(150 \mathrm{MHz}, \mathrm{CDCl}_{3}\right) \delta 181.2,157.9,136.8,130.1,126.8,113.6,112.4,55.4,40.1,30.7$, 28.8, 25.7.

HRMS (ESI-TOF) Calcd for $\mathrm{C}_{12} \mathrm{H}_{13} \mathrm{O}_{3}{ }^{-}[\mathrm{M}-\mathrm{H}]^{-}: 205.0870$; found: 205.0869.<smiles>COc1cccc2c1CCC(C(=O)O)C2</smiles>

5-Methoxy-1,2,3,4-tetrahydronaphthalene-2-carboxylic acid (2k) 
Following General Procedure A on $0.1 \mathrm{mmol}$ scale. Purification by pTLC afforded the title compound (colorless oil, $7.3 \mathrm{mg}, 35 \%$ yield).

${ }^{1} \mathrm{H}$ NMR $\left(600 \mathrm{MHz}, \mathrm{CDCl}_{3}\right) \delta 7.10(\mathrm{t}, J=7.9 \mathrm{~Hz}, 1 \mathrm{H}), 6.72(\mathrm{~d}, J=7.7 \mathrm{~Hz}, 1 \mathrm{H}), 6.67(\mathrm{~d}, J=8.1$ $\mathrm{Hz}, 1 \mathrm{H}), 3.82$ (s, 3H), $3.18-3.08(\mathrm{~m}, 1 \mathrm{H}), 2.93-2.80(\mathrm{~m}, 2 \mathrm{H}), 2.79-2.70(\mathrm{~m}, 2 \mathrm{H}), 2.25-2.18$ (m, 1H), $1.92-1.78(\mathrm{~m}, 1 \mathrm{H})$.

${ }^{13} \mathrm{C} \mathrm{NMR}\left(150 \mathrm{MHz}, \mathrm{CDCl}_{3}\right) \delta 179.1,157.5,137.1,126.4,123.7,121.1,107.2,55.4,39.4,28.7$, 25.6, 25.4.

HRMS (ESI-TOF) Calcd for $\mathrm{C}_{12} \mathrm{H}_{13} \mathrm{O}_{3}{ }^{-}$[M-H]": 205.0870; found: 205.0869.<smiles>O=C(O)C1CCc2ccc(F)cc2C1</smiles>

\section{7-Fluoro-1,2,3,4-tetrahydronaphthalene-2-carboxylic acid (21)}

Following General Procedure A on $0.1 \mathrm{mmol}$ scale. Purification by pTLC afforded the title compound (colorless oil, $10.1 \mathrm{mg}, 52 \%$ yield).

${ }^{1} \mathrm{H}$ NMR (600 MHz, $\left.\mathrm{CDCl}_{3}\right) \delta 7.12-6.99(\mathrm{~m}, 1 \mathrm{H}), 6.90-6.75(\mathrm{~m}, 2 \mathrm{H}), 3.09-2.93(\mathrm{~m}, 2 \mathrm{H}), 2.93$ $-2.75(\mathrm{~m}, 3 \mathrm{H}), 2.29-2.19(\mathrm{~m}, 1 \mathrm{H}), 1.96-1.84(\mathrm{~m}, 1 \mathrm{H})$.

${ }^{13} \mathrm{C}$ NMR $\left(150 \mathrm{MHz}, \mathrm{CDCl}_{3}\right)$ (major rotamer) $\delta 180.7,161.2(\mathrm{~d}, J=243.7 \mathrm{~Hz}), 136.6(\mathrm{~d}, J=7.4$ $\mathrm{Hz}), 131.2(\mathrm{~d}, J=2.7 \mathrm{~Hz}), 130.3(\mathrm{~d}, J=8.2 \mathrm{~Hz}), 115.3(\mathrm{~d}, J=20.6 \mathrm{~Hz}), 113.3(\mathrm{~d}, J=21.4 \mathrm{~Hz})$, $39.5,31.4,27.8,25.8$.

${ }^{13} \mathrm{C}$ NMR $\left(150 \mathrm{MHz}, \mathrm{CDCl}_{3}\right)$ (minor rotamer) $\delta 180.8,161.3(\mathrm{~d}, J=244.2 \mathrm{~Hz}), 137.6(\mathrm{~d}, J=7.3$ $\mathrm{Hz}), 130.5(\mathrm{~d}, J=7.8 \mathrm{~Hz}), 130.2(\mathrm{~d}, J=2.8 \mathrm{~Hz}), 115.1(\mathrm{~d}, J=20.7 \mathrm{~Hz}), 113.2(\mathrm{~d}, J=21.1 \mathrm{~Hz})$, 39.7, 30.8, 28.6, 25.4 .

HRMS (ESI-TOF) Calcd for $\mathrm{C}_{11} \mathrm{H}_{10} \mathrm{FO}_{2}^{-}[\mathrm{M}-\mathrm{H}]^{-}:$193.0670; found: 193.0666 .

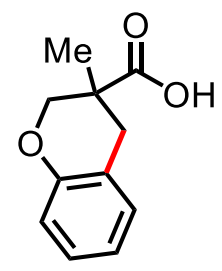

\section{3-Methylchromane-3-carboxylic acid (2m)}

Following General Procedure A on $0.1 \mathrm{mmol}$ scale. Purification by pTLC afforded the title compound (colorless oil, $13.0 \mathrm{mg}, 68 \%$ yield). 
${ }^{1} \mathrm{H}$ NMR $\left(600 \mathrm{MHz}, \mathrm{CDCl}_{3}\right) \delta 7.15-7.08(\mathrm{~m}, 1 \mathrm{H}), 7.06(\mathrm{~d}, J=7.4 \mathrm{~Hz}, 1 \mathrm{H}), 6.91-6.85(\mathrm{~m}, 1 \mathrm{H})$, $6.83(\mathrm{~d}, J=8.2 \mathrm{~Hz}, 1 \mathrm{H}), 4.31(\mathrm{dd}, J=10.8,1.4 \mathrm{~Hz}, 1 \mathrm{H}), 3.95(\mathrm{~d}, J=10.8 \mathrm{~Hz}, 1 \mathrm{H}), 3.27(\mathrm{~d}, J=$ $16.4 \mathrm{~Hz}, 1 \mathrm{H}), 2.70(\mathrm{~d}, J=16.4 \mathrm{~Hz}, 1 \mathrm{H}), 1.34$ (s, 3H).

${ }^{13} \mathrm{C} \mathrm{NMR}\left(150 \mathrm{MHz}, \mathrm{CDCl}_{3}\right) \delta 180.7,153.5,130.0,127.7,121.1,120.1,116.8,71.0,40.8,34.5$, 21.1 .

HRMS (ESI-TOF) Calcd for $\mathrm{C}_{11} \mathrm{H}_{11} \mathrm{O}_{3}^{-}[\mathrm{M}-\mathrm{H}]^{-}: 191.0714$; found: 191.0713.

The NMR data matches the reported data ${ }^{12}$.

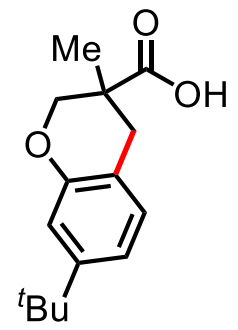

7-(tert-Butyl)-3-methylchromane-3-carboxylic acid (2n)

Following General Procedure A on $0.1 \mathrm{mmol}$ scale. Purification by pTLC afforded the title compound (colorless oil, $20.0 \mathrm{mg}, 80 \%$ yield, $\mathbf{2 n} / \mathbf{2 n} \mathbf{n}^{\prime}=3 / 1$ ).

${ }^{1} \mathrm{H}$ NMR $\left(600 \mathrm{MHz}, \mathrm{CDCl}_{3}\right) \delta 6.99(\mathrm{~d}, J=8.0 \mathrm{~Hz}, 1 \mathrm{H}), 6.92(\mathrm{dd}, J=8.0,2.0 \mathrm{~Hz}, 1 \mathrm{H}), 6.86(\mathrm{~d}, J$ $=2.0 \mathrm{~Hz}, 1 \mathrm{H}), 4.29(\mathrm{dd}, J=10.8,1.4 \mathrm{~Hz}, 1 \mathrm{H}), 3.93(\mathrm{dd}, J=10.8,1.4 \mathrm{~Hz}, 1 \mathrm{H}), 3.24(\mathrm{~d}, J=16.3$ $\mathrm{Hz}, 1 \mathrm{H}), 2.66$ (d, $J=16.3 \mathrm{~Hz}, 1 \mathrm{H}), 1.34$ (s, 3H), 1.28 (s, 9H).

${ }^{13} \mathrm{C}$ NMR $\left(150 \mathrm{MHz}, \mathrm{CDCl}_{3}\right) \delta 180.8,153.0,151.2,129.4,118.4,117.0,113.7,71.0,40.9,34.6$, 34.1, 31.4, 21.2.

HRMS (ESI-TOF) Calcd for $\mathrm{C}_{15} \mathrm{H}_{19} \mathrm{O}_{3}{ }^{-}[\mathrm{M}-\mathrm{H}]^{-}: 247.1340$; found: 247.1339.

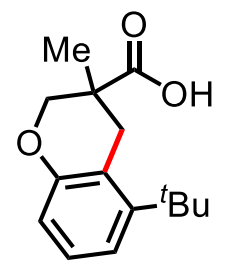

\section{5-(tert-Butyl)-3-methylchromane-3-carboxylic acid (2n')}

${ }^{1} \mathrm{H}$ NMR $\left(600 \mathrm{MHz}, \mathrm{CDCl}_{3}\right) \delta 7.05(\mathrm{t}, J=7.8 \mathrm{~Hz}, 1 \mathrm{H}), 6.99(\mathrm{~d}, J=7.8 \mathrm{~Hz}, 1 \mathrm{H}), 6.73(\mathrm{~d}, J=7.8$ $\mathrm{Hz}, 1 \mathrm{H}), 4.37$ (d, $J=10.5 \mathrm{~Hz}, 1 \mathrm{H}), 3.91(\mathrm{~d}, J=10.5 \mathrm{~Hz}, 1 \mathrm{H}), 3.51$ (d, $J=16.0 \mathrm{~Hz}, 1 \mathrm{H}), 2.90(\mathrm{~d}, J$ $=16.0 \mathrm{~Hz}, 1 \mathrm{H}), 1.42(\mathrm{~s}, 9 \mathrm{H}), 1.35(\mathrm{~s}, 3 \mathrm{H})$.

${ }^{13} \mathrm{C} \mathrm{NMR}\left(150 \mathrm{MHz}, \mathrm{CDCl}_{3}\right) \delta 180.9,154.0,149.4,127.1,119.0,118.9,115.6,70.4,40.8,36.2$, $34.9,31.2,21.5$. 
HRMS (ESI-TOF) Calcd for $\mathrm{C}_{15} \mathrm{H}_{19} \mathrm{O}_{3}{ }^{-}$[M-H] $]^{-2}$ 247.1340; found: 247.1337.

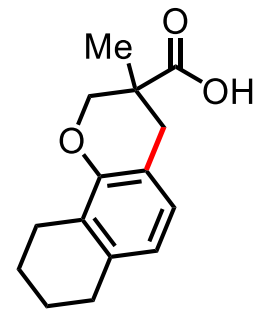

\section{3-Methyl-3,4,7,8,9,10-hexahydro-2H-benzo[ $h]$ chromene-3-carboxylic acid (2o)}

Following General Procedure A on $0.1 \mathrm{mmol}$ scale. Purification by pTLC afforded the title compound (colorless oil, $21.0 \mathrm{mg}, 85 \%$ yield).

${ }^{1} \mathrm{H}$ NMR $\left(600 \mathrm{MHz}, \mathrm{CDCl}_{3}\right) \delta 6.81(\mathrm{~d}, J=7.8 \mathrm{~Hz}, 1 \mathrm{H}), 6.63(\mathrm{~d}, J=7.8 \mathrm{~Hz}, 1 \mathrm{H}), 4.29(\mathrm{~d}, J=10.8$ $\mathrm{Hz}, 1 \mathrm{H}), 3.96(\mathrm{~d}, J=10.8 \mathrm{~Hz}, 1 \mathrm{H}), 3.23(\mathrm{~d}, J=16.3 \mathrm{~Hz}, 1 \mathrm{H}), 2.70(\mathrm{t}, J=5.8 \mathrm{~Hz}, 2 \mathrm{H}), 2.65$ (d, $J=$ $16.3 \mathrm{~Hz}, 1 \mathrm{H}), 2.64-2.58(\mathrm{~m}, 2 \mathrm{H}), 1.80-1.69$ (m, 4H), 1.33 (s, 3H).

${ }^{13} \mathrm{C}$ NMR $\left(150 \mathrm{MHz}, \mathrm{CDCl}_{3}\right) \delta 181.2,151.1,136.7,126.5,125.4,121.6,116.2,70.9,40.7,34.5$, 29.6, 23.1, 23.0, 22.9, 21.1.

HRMS (ESI-TOF) Calcd for $\mathrm{C}_{15} \mathrm{H}_{17} \mathrm{O}_{3}^{-}$[M-H]": 245.1183; found: 245.1183 .<smiles>CC1(C(=O)O)COc2c(Br)cccc2C1</smiles>

\section{8-Benzyl-3-methylchromane-3-carboxylic acid (2p)}

Following General Procedure A on $0.1 \mathrm{mmol}$ scale. Purification by pTLC afforded the title compound (colorless oil, $20.0 \mathrm{mg}, 70 \%$ yield).

${ }^{1} \mathrm{H}$ NMR $\left(600 \mathrm{MHz}, \mathrm{CDCl}_{3}\right) \delta 7.27-7.20(\mathrm{~m}, 2 \mathrm{H}), 7.19(\mathrm{~d}, J=7.5 \mathrm{~Hz}, 2 \mathrm{H}), 7.15(\mathrm{t}, J=7.3 \mathrm{~Hz}$, $1 \mathrm{H}), 6.94(\mathrm{~d}, J=7.5 \mathrm{~Hz}, 1 \mathrm{H}), 6.89$ (d, $J=7.4 \mathrm{~Hz}, 1 \mathrm{H}), 6.80(\mathrm{t}, J=7.5 \mathrm{~Hz}, 1 \mathrm{H}), 4.31(\mathrm{~d}, J=10.7$ $\mathrm{Hz}, 1 \mathrm{H}), 4.03-3.88$ (m, 3H), 3.28 (d, $J=16.4 \mathrm{~Hz}, 1 \mathrm{H}), 2.71$ (d, $J=16.4 \mathrm{~Hz}, 1 \mathrm{H}), 1.34$ (s, 3H). ${ }^{13} \mathrm{C}$ NMR $\left(150 \mathrm{MHz}, \mathrm{CDCl}_{3}\right) \delta 180.5,151.2,141.1,129.1,129.0,128.5,128.4,128.1,125.9,120.7$, 119.9, 71.0, 40.7, 35.7, 34.7, 21.0.

HRMS (ESI-TOF) Calcd for $\mathrm{C}_{18} \mathrm{H}_{17} \mathrm{O}_{3}^{-}$[M-H]": 281.1183; found: 281.1184 . 
<smiles>CC1(C(=O)O)COc2c(Br)cccc2C1</smiles>

\section{8-Bromo-3-methylchromane-3-carboxylic acid (2q)}

Following General Procedure A on $0.1 \mathrm{mmol}$ scale. Purification by pTC afforded the title compound (colorless oil, $8.5 \mathrm{mg}, 31 \%$ yield).

${ }^{1} \mathrm{H}$ NMR $\left(600 \mathrm{MHz}, \mathrm{CDCl}_{3}\right) \delta 7.36(\mathrm{~d}, J=7.8 \mathrm{~Hz}, 1 \mathrm{H}), 7.02(\mathrm{~d}, J=7.8 \mathrm{~Hz}, 1 \mathrm{H}), 6.76(\mathrm{t}, J=7.8$ $\mathrm{Hz}, 1 \mathrm{H}), 4.41(\mathrm{~d}, J=10.8 \mathrm{~Hz}, 1 \mathrm{H}), 4.07$ (d, $J=10.8 \mathrm{~Hz}, 1 \mathrm{H}), 3.29$ (d, $J=16.4 \mathrm{~Hz}, 1 \mathrm{H}), 2.72(\mathrm{~d}, J$ $=16.4 \mathrm{~Hz}, 1 \mathrm{H}), 1.36(\mathrm{~s}, 3 \mathrm{H})$.

${ }^{13} \mathrm{C}$ NMR $\left(150 \mathrm{MHz}, \mathrm{CDCl}_{3}\right) \delta 179.8,150.1,131.5,129.2,121.9,110.9,71.7,40.7,34.6,21.0(1$ carbon signal was not assigned due to overlaps).

HRMS (ESI-TOF) Calcd for $\mathrm{C}_{11} \mathrm{H}_{10} \mathrm{BrO}_{3}{ }^{-}[\mathrm{M}-\mathrm{H}]^{-}:$: 268.9819; found: 268.9820 .<smiles>CC1(C(=O)O)COc2c(cccc2C(F)(F)F)CO1</smiles>

\section{3-Methyl-8-(trifluoromethyl)chromane-3-carboxylic acid (2r)}

Following General Procedure A on $0.1 \mathrm{mmol}$ scale. Purification by pTLC afforded the title compound (colorless oil, $4.5 \mathrm{mg}, 17 \%$ yield).

${ }^{1} \mathrm{H}$ NMR $\left(600 \mathrm{MHz}, \mathrm{CDCl}_{3}\right) \delta 7.40(\mathrm{~d}, J=7.8 \mathrm{~Hz}, 1 \mathrm{H}), 7.24(\mathrm{~d}, J=7.8 \mathrm{~Hz}, 1 \mathrm{H}), 6.93(\mathrm{t}, J=7.8$ $\mathrm{Hz}, 1 \mathrm{H}), 4.41(\mathrm{~d}, J=10.9 \mathrm{~Hz}, 1 \mathrm{H}), 4.08(\mathrm{~d}, J=10.9 \mathrm{~Hz}, 1 \mathrm{H}), 3.31$ (d, $J=16.4 \mathrm{~Hz}, 1 \mathrm{H}), 2.75(\mathrm{~d}, J$ $=16.4 \mathrm{~Hz}, 1 \mathrm{H}), 1.38(\mathrm{~s}, 3 \mathrm{H})$.

${ }^{13} \mathrm{C} \mathrm{NMR}\left(150 \mathrm{MHz}, \mathrm{CDCl}_{3}\right) \delta 179.5,151.6,133.8,125.4(\mathrm{q}, J=5.4 \mathrm{~Hz}), 123.7(\mathrm{q}, J=272.3 \mathrm{~Hz})$, 121.6, 120.2, 118.2 (q, $J=30.9 \mathrm{~Hz}), 71.2,40.3,34.3,21.0$.

HRMS (ESI-TOF) Calcd for $\mathrm{C}_{12} \mathrm{H}_{10} \mathrm{~F}_{3} \mathrm{O}_{3}{ }^{-}$[M-H] $]^{-}$: 259.0588; found: 259.0587.<smiles>COc1ccc2c(c1)OC[C@@H](C(=O)O)C2</smiles>

(R)-7-Methoxychromane-3-carboxylic acid (2s) 
Following General Procedure A on $0.1 \mathrm{mmol}$ scale. Purification by pTLC afforded the title compound (colorless oil, $15.0 \mathrm{mg}, 72 \%$ yield).

${ }^{1} \mathrm{H}$ NMR (600 MHz, $\left.\mathrm{CDCl}_{3}\right) \delta 6.98(\mathrm{~d}, J=8.4 \mathrm{~Hz}, 1 \mathrm{H}), 6.49(\mathrm{dd}, J=8.4,2.6 \mathrm{~Hz}, 1 \mathrm{H}), 6.39(\mathrm{~d}, J$ $=2.6 \mathrm{~Hz}, 1 \mathrm{H}), 4.47-4.40(\mathrm{~m}, 1 \mathrm{H}), 4.21-4.14(\mathrm{~m}, 1 \mathrm{H}), 3.75(\mathrm{~s}, 3 \mathrm{H}), 3.10-3.04(\mathrm{~m}, 1 \mathrm{H}), 3.03-$ $2.96(\mathrm{~m}, 2 \mathrm{H})$.

${ }^{13} \mathrm{C} \mathrm{NMR}\left(150 \mathrm{MHz}, \mathrm{CDCl}_{3}\right) \delta 176.8,159.4,154.8,130.3,112.1,108.1,101.7,66.3,55.5,38.4$, 26.8 .

HRMS (ESI-TOF) Calcd for $\mathrm{C}_{11} \mathrm{H}_{11} \mathrm{O}_{4}{ }^{-}$[M-H] : 207.0663; found: 207.0660.<smiles>CCC1(C(=O)O)Cc2ccccc2C1</smiles>

\section{2-Ethyl-2,3-dihydro-1H-indene-2-carboxylic acid (2u)}

Following General Procedure A on $0.1 \mathrm{mmol}$ scale. Purification by pTLC afforded the title compound (colorless oil, $10.0 \mathrm{mg}$, 53\% yield).

${ }^{1} \mathrm{H}$ NMR (600 MHz, CDCl $) \delta 7.21-7.16(\mathrm{~m}, 2 \mathrm{H}), 7.16-7.11(\mathrm{~m}, 2 \mathrm{H}), 3.48(\mathrm{~d}, J=16.2 \mathrm{~Hz}, 2 \mathrm{H})$, $2.92(\mathrm{~d}, J=16.2 \mathrm{~Hz}, 2 \mathrm{H}), 1.83(\mathrm{q}, J=7.2 \mathrm{~Hz}, 2 \mathrm{H}), 0.94(\mathrm{t}, J=7.2 \mathrm{~Hz}, 3 \mathrm{H})$.

${ }^{13} \mathrm{C}$ NMR $\left(150 \mathrm{MHz}, \mathrm{CDCl}_{3}\right) \delta 182.3,141.4,126.7,124.6,54.7,41.8,31.5,10.0$.

HRMS (ESI-TOF) Calcd for $\mathrm{C}_{12} \mathrm{H}_{13} \mathrm{O}_{2}{ }^{-}$[M-H] : 189.0921; found: 189.0918.<smiles>Cc1cccc2c1CC(C)(C(=O)O)C2</smiles>

\section{2,4-Dimethyl-2,3-dihydro-1H-indene-2-carboxylic acid (2v)}

Following General Procedure B on $0.1 \mathrm{mmol}$ scale. Purification by pTLC afforded the title compound (colorless oil, $11.5 \mathrm{mg}, 61 \%$ yield).

${ }^{1} \mathrm{H}$ NMR $\left(600 \mathrm{MHz}, \mathrm{CDCl}_{3}\right) \delta 7.08(\mathrm{t}, J=7.4 \mathrm{~Hz}, 1 \mathrm{H}), 7.03(\mathrm{~d}, J=7.4 \mathrm{~Hz}, 1 \mathrm{H}), 6.98(\mathrm{~d}, J=7.4$ $\mathrm{Hz}, 1 \mathrm{H}), 3.53(\mathrm{~d}, J=15.9 \mathrm{~Hz}, 1 \mathrm{H}), 3.43(\mathrm{~d}, J=16.0 \mathrm{~Hz}, 1 \mathrm{H}), 2.86(\mathrm{~d}, J=15.9 \mathrm{~Hz}, 1 \mathrm{H}), 2.80(\mathrm{~d}, J$ $=16.0 \mathrm{~Hz}, 1 \mathrm{H}), 2.24(\mathrm{~s}, 3 \mathrm{H}), 1.41(\mathrm{~s}, 3 \mathrm{H})$.

${ }^{13} \mathrm{C}$ NMR $\left(150 \mathrm{MHz}, \mathrm{CDCl}_{3}\right) \delta 184.2,141.0,140.0,134.2,127.6,127.0,122.1,49.0,44.2,42.8$, 25.4, 19.2.

HRMS (ESI-TOF) Calcd for $\mathrm{C}_{12} \mathrm{H}_{13} \mathrm{O}_{2}^{-}[\mathrm{M}-\mathrm{H}]^{-}:$189.0921; found: 189.0915 . 


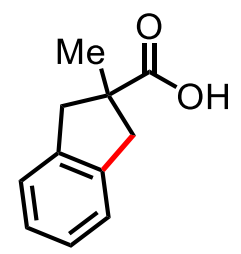

2-Methyl-2,3-dihydro-1H-indene-2-carboxylic acid (2w)

Following General Procedure B on $0.1 \mathrm{mmol}$ scale. Purification by pTLC afforded the title compound (colorless oil, $8.0 \mathrm{mg}, 48 \%$ yield).

${ }^{1} \mathrm{H}$ NMR $\left(600 \mathrm{MHz}, \mathrm{CDCl}_{3}\right) \delta 7.23-7.18(\mathrm{~m}, 2 \mathrm{H}), 7.18-7.14(\mathrm{~m}, 2 \mathrm{H}), 3.52(\mathrm{~d}, J=15.8 \mathrm{~Hz}, 2 \mathrm{H})$, $2.85(\mathrm{~d}, J=15.8 \mathrm{~Hz}, 2 \mathrm{H}), 1.41(\mathrm{~s}, 3 \mathrm{H})$.

${ }^{13} \mathrm{C}$ NMR $\left(150 \mathrm{MHz}, \mathrm{CDCl}_{3}\right) \delta 182.5,141.2,126.8,124.8,49.5,44.0,25.0$.

HRMS (ESI-TOF) Calcd for $\mathrm{C}_{11} \mathrm{H}_{11} \mathrm{O}_{2}^{-}[\mathrm{M}-\mathrm{H}]^{-}:$175.0765; found: 175.0762 .

The NMR data matches the reported data ${ }^{13}$. 
Total synthesis of ( \pm )-russujaponol F

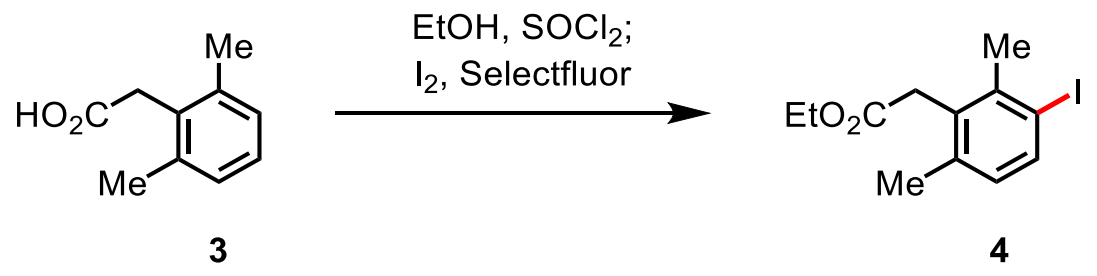

To the $\mathrm{EtOH}(5.0 \mathrm{~mL})$ solution of $\mathbf{3}(1.0 \mathrm{mmol}, 164 \mathrm{mg})$ was added $\mathrm{SOCl}_{2}(2.0$ equiv, $0.15 \mathrm{~mL})$ at $0{ }^{\circ} \mathrm{C}$ and then the mixture was stirred under reflux overnight. After being allowed to cool to room temperature, the mixture was concentrated in vacuo to afford the corresponding ethyl ester. Following literature procedure ${ }^{10}$ with slight modification, to the $\mathrm{CH}_{3} \mathrm{CN}$ solution $(10.0 \mathrm{~mL})$ of the ethyl ester was added $\mathrm{I}_{2}(0.5$ equiv, $127 \mathrm{mg})$ and Selectfluor $(0.5$ equiv, $177 \mathrm{mg})$ and the mixture was stirred at $60{ }^{\circ} \mathrm{C}$ for $3 \mathrm{~h}$. After being allowed to cool to room temperature, the mixture was diluted with EA, washed with saturated $\mathrm{Na}_{2} \mathrm{~S}_{2} \mathrm{O}_{3}$, and concentrated in vacuo. The crude mixture was purified by column chromatography to afford the iodination product 4 ( $250 \mathrm{mg}, 79 \%$ yield).<smiles>CCOC(=O)Cc1c(C)ccc(I)c1[N+](=O)[O-]</smiles>

\section{Ethyl 2-(3-iodo-2,6-dimethylphenyl)acetate (4)}

${ }^{1} \mathrm{H} \mathrm{NMR}\left(600 \mathrm{MHz}, \mathrm{CDCl}_{3}\right) \delta 7.65(\mathrm{~d}, J=8.1 \mathrm{~Hz}, 1 \mathrm{H}), 6.74(\mathrm{~d}, J=8.1 \mathrm{~Hz}, 1 \mathrm{H}), 4.15(\mathrm{q}, J=7.1$ $\mathrm{Hz}, 2 \mathrm{H}), 3.75$ (s, 2H), 2.48 (s, 3H), 2.29 (s, 3H), 1.25 (t, $J=7.1 \mathrm{~Hz}, 3 \mathrm{H})$.

${ }^{13} \mathrm{C}$ NMR $\left(150 \mathrm{MHz}, \mathrm{CDCl}_{3}\right) \delta 171.0,139.9,138.1,137.8,133.0,129.8,99.7,61.1,37.1,26.0$, 20.5, 14.3.

HRMS (ESI-TOF) Calcd for $\mathrm{C}_{12} \mathrm{H}_{16} \mathrm{IO}_{2}{ }^{+}[\mathrm{M}+\mathrm{H}]^{+}: 319.0189$; found: 319.0196. 


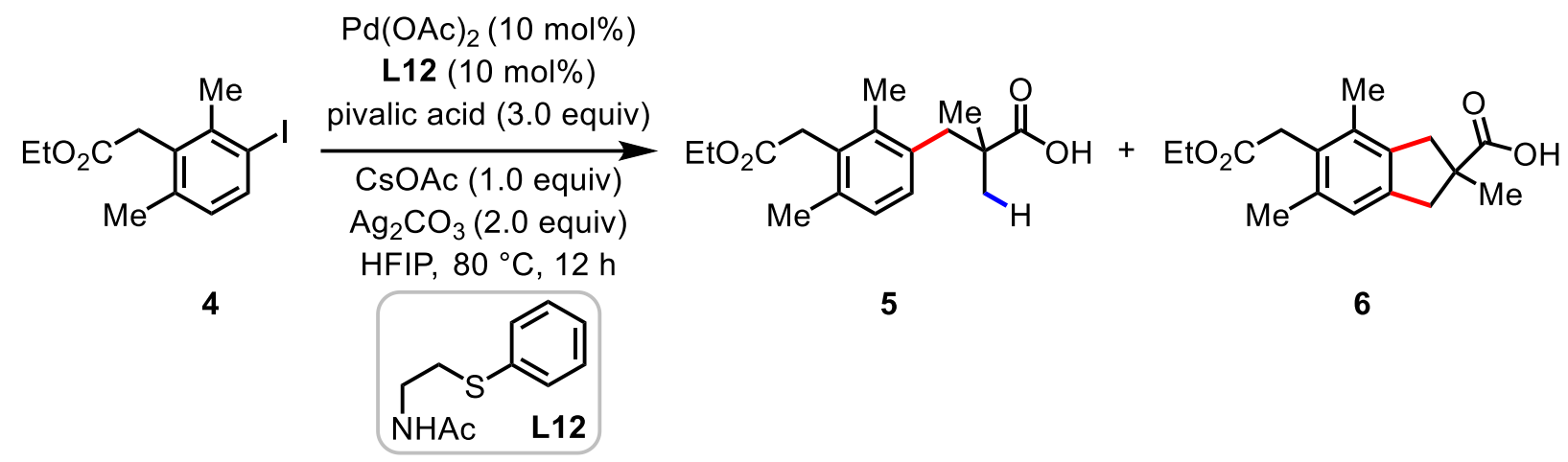

In the culture tube, $\mathrm{Pd}(\mathrm{OAc})_{2}(10 \mathrm{~mol} \%, 2.2 \mathrm{mg})$, ligand $\mathbf{L 1 2}(10 \mathrm{~mol} \%, 2.0 \mathrm{mg})$, CsOAc (1.0 equiv, $19.2 \mathrm{mg}$ ), $\mathrm{Ag}_{2} \mathrm{CO}_{3}$ (2.0 equiv, $55.1 \mathrm{mg}$ ), pivalic acid (3.0 equiv, $30.6 \mathrm{mg}$ ) and 4 (0.1 mmol, $31.8 \mathrm{mg})$ in order were weighed in air and placed with a magnetic stir bar. Then HFIP (1.0 mL) was added. The reaction mixture was stirred at $\mathrm{rt}$ for $3 \mathrm{~min}$, and then heated to $80^{\circ} \mathrm{C}$ for $12 \mathrm{~h}(600$ rpm). After being allowed to cool to room temperature, the mixture was treated with $\mathrm{HCO}_{2} \mathrm{H}(0.1$ $\mathrm{mL}$ ), diluted with DCM, filtered through a Celite plug, and concentrated in vacuo. The crude mixture was purified by pTLC (hexane/EA) to afford the arylation product 5 (18.0 $\mathrm{mg}, 62 \%$ yield) and the product 6 ( $3.5 \mathrm{mg}, 12 \%$ yield).<smiles>[M]C(C)(Cc1ccc(C)c(CC(=O)OCC)c1C)C(=O)O</smiles>

\section{3-(3-(2-Ethoxy-2-oxoethyl)-2,4-dimethylphenyl)-2,2-dimethylpropanoic acid (5)}

${ }^{1} \mathrm{H}$ NMR $\left(600 \mathrm{MHz}, \mathrm{CDCl}_{3}\right) \delta 6.99(\mathrm{~d}, J=7.9 \mathrm{~Hz}, 1 \mathrm{H}), 6.96(\mathrm{~d}, J=7.9 \mathrm{~Hz}, 1 \mathrm{H}), 4.14(\mathrm{q}, J=7.1$ $\mathrm{Hz}, 2 \mathrm{H}$ ), 3.70 (s, 2H), 2.99 (s, 2H), 2.30 (s, 3H), 2.26 (s, 3H), 1.23 (t, J = 7.1 Hz, 3H), 1.19 (s, 6H). ${ }^{13} \mathrm{C} \mathrm{NMR}\left(150 \mathrm{MHz}, \mathrm{CDCl}_{3}\right) \delta 183.1,171.6,136.5,135.7,134.0,132.5,130.1,127.5,60.9,44.1$, $42.3,36.2,27.3,24.7,20.7,17.0,14.4$.

HRMS (ESI-TOF) Calcd for $\mathrm{C}_{17} \mathrm{H}_{23} \mathrm{O}_{4}^{-}$[M-H]": 291.1602; found: 291.1605 . 
<smiles>CCOC(=O)Cc1c(C)ccc(CC(C)(C)C(=O)O)c1C</smiles>

5

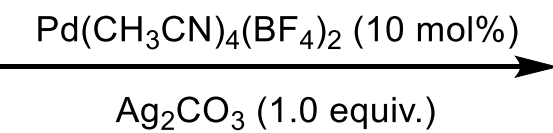

$\left[\mathrm{F}^{+}\right]$(2.0 equiv.) HFIP, $90^{\circ} \mathrm{C}, 12 \mathrm{~h}$<smiles>CCOC(=O)Cc1c(C)cc2c(c1C)CC(C)(C(=O)O)C2</smiles>

6

In the culture tube, $\mathrm{Pd}\left(\mathrm{CH}_{3} \mathrm{CN}\right)_{4}\left(\mathrm{BF}_{4}\right)_{2}(10 \mathrm{~mol} \%, 2.2 \mathrm{mg}), \mathrm{Ag}_{2} \mathrm{CO}_{3}(1.0$ equiv, $13.8 \mathrm{mg})$, 1-fluoro2,4,6-trimethylpyridinium tetrafluoroborate ( 2.0 equiv, $22.7 \mathrm{mg}$ ), and $5(0.05 \mathrm{mmol}, 14.6 \mathrm{mg}$ ) in order were weighed in air and placed with a magnetic stir bar. Then HFIP $(0.5 \mathrm{~mL})$ was added. The reaction mixture was stirred at $\mathrm{rt}$ for $3 \mathrm{~min}$, and then heated to $90{ }^{\circ} \mathrm{C}$ for $12 \mathrm{~h}(600 \mathrm{rpm})$. After being allowed to cool to room temperature, the mixture was treated with $\mathrm{HCO}_{2} \mathrm{H}(0.05 \mathrm{~mL})$, diluted with DCM, filtered through a Celite plug, and concentrated in vacuo. The crude mixture was purified by pTLC (hexane/EA) to afford the product 6 ( $6.0 \mathrm{mg}, 41 \%$ yield).<smiles>CCOCCc1c(C)cc2c(c1C)CC(C)(C(=O)O)C2</smiles>

\section{5-(2-Ethoxy-2-oxoethyl)-2,4,6-trimethyl-2,3-dihydro-1H-indene-2-carboxylic acid (6)}

${ }^{1} \mathrm{H}$ NMR $\left(600 \mathrm{MHz}, \mathrm{CDCl}_{3}\right) \delta 6.90(\mathrm{~s}, 1 \mathrm{H}), 4.14(\mathrm{q}, J=7.0 \mathrm{~Hz}, 2 \mathrm{H}), 3.66(\mathrm{~s}, 2 \mathrm{H}), 3.49$ (d, $J=16.0$ $\mathrm{Hz}, 1 \mathrm{H}), 3.44(\mathrm{~d}, J=16.0 \mathrm{~Hz}, 1 \mathrm{H}), 2.81(\mathrm{~d}, J=16.0 \mathrm{~Hz}, 1 \mathrm{H}), 2.80(\mathrm{~d}, J=16.0 \mathrm{~Hz}, 1 \mathrm{H}), 2.30$ (s, $3 \mathrm{H}), 2.21(\mathrm{~s}, 3 \mathrm{H}), 1.41(\mathrm{~s}, 3 \mathrm{H}), 1.25(\mathrm{t}, J=7.1 \mathrm{~Hz}, 3 \mathrm{H})$.

${ }^{13} \mathrm{C}$ NMR $\left(150 \mathrm{MHz}, \mathrm{CDCl}_{3}\right) \delta 181.9,171.7,139.7,138.3,136.0,133.3,130.0,124.1,60.9,48.8$, 44.2, 43.5, 35.4, 25.5, 20.8, 16.5, 14.4 .

HRMS (ESI-TOF) Calcd for $\left.\mathrm{C}_{17} \mathrm{H}_{21} \mathrm{O}_{4}{ }^{-}[\mathrm{M}-\mathrm{H}]\right]^{-}: 289.1445$; found: 289.1447 . 


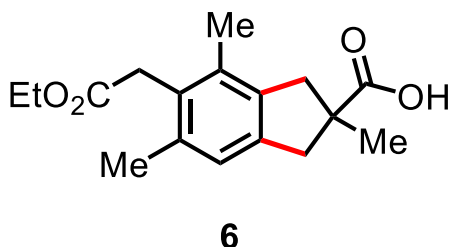

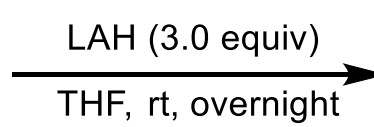

6

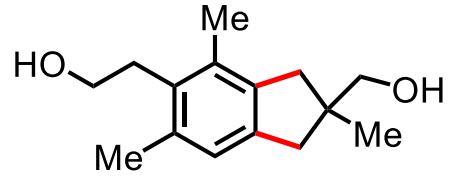

(士)-russujaponol F

In the culture tube, to the THF $(1.0 \mathrm{~mL})$ solution of $6(0.02 \mathrm{mmol}, 6.0 \mathrm{mg})$ was added LAH (3.0 equiv, $1.0 \mathrm{M}$ in THF, $0.06 \mathrm{~mL}$ ) at $0{ }^{\circ} \mathrm{C}$. The reaction mixture was warmed to $\mathrm{rt}$ and stirred at $\mathrm{rt}$ overnight. The mixture was diluted with ether, washed with saturated $\mathrm{NH}_{4} \mathrm{Cl}$, and concentrated in vacuo. The crude mixture was purified by pTLC (hexane/EA) to afford the ( \pm )-russujaponol F (4.5 mg, $96 \%$ yield). The NMR data matches the reported data ${ }^{14,15}$.

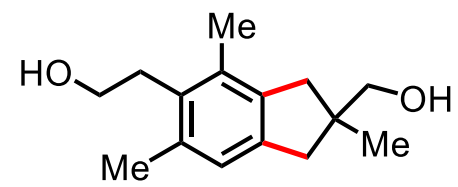

\section{(士)-Russujaponol F}

${ }^{1} \mathrm{H}$ NMR $\left(600 \mathrm{MHz}, \mathrm{CDCl}_{3}\right) \delta 6.87(\mathrm{~s}, 1 \mathrm{H}), 3.74(\mathrm{t}, J=7.4 \mathrm{~Hz}, 2 \mathrm{H}), 3.52(\mathrm{~s}, 2 \mathrm{H}), 2.95(\mathrm{t}, J=7.5$ Hz, 2H), $\delta 2.88(\mathrm{~d}, J=15.9 \mathrm{~Hz}, 1 \mathrm{H}), 2.84(\mathrm{~d}, J=15.9 \mathrm{~Hz}, 1 \mathrm{H}), 2.63$ (d, $J=15.9 \mathrm{~Hz}, 1 \mathrm{H}), 2.59$ (d, $J=15.9 \mathrm{~Hz}, 1 \mathrm{H}), 2.32(\mathrm{~s}, 3 \mathrm{H}), 2.22(\mathrm{~s}, 3 \mathrm{H}), 1.18(\mathrm{~s}, 3 \mathrm{H})$.

${ }^{13} \mathrm{C} \mathrm{NMR}\left(150 \mathrm{MHz}, \mathrm{CDCl}_{3}\right) \delta 140.3,139.8,135.4,133.2,132.3,124.4,71.1,62.1,44.3,43.1$, 42.4, 32.9, 24.6, 20.6, 16.3.

HRMS (ESI-TOF) Calcd for $\mathrm{C}_{15} \mathrm{H}_{21} \mathrm{O}_{2}^{-}[\mathrm{M}-\mathrm{H}]^{-}: 233.1547$; found: 233.1544 . 


\section{KIE experiments}<smiles>CCC(C)(COc1ccccc1)C(=O)O</smiles>

$1 \mathrm{~m}$<smiles>[2H]c1c([2H])c([2H])c(OCC(C)(C)C(=O)O)c([2H])c1[2H]</smiles>

$1 \mathrm{~m}-\mathrm{d}_{5}$
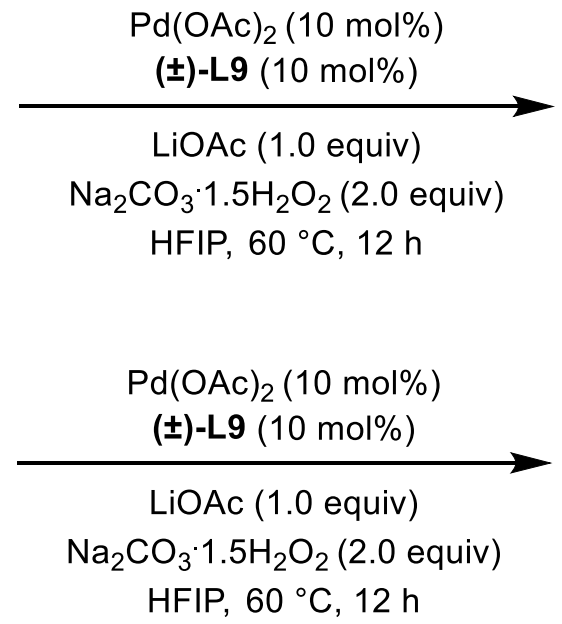

$$
k_{\mathrm{H}} / k_{\mathrm{D}}=1.1
$$
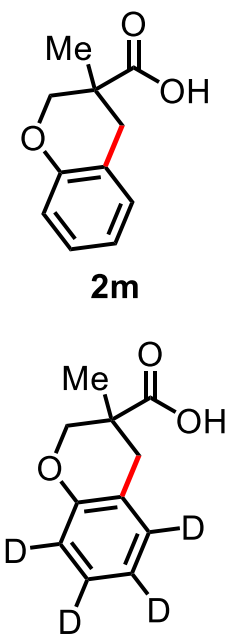

$2 \mathrm{~m}-\mathrm{d}_{4}$

Following General Procedure A on $0.05 \mathrm{mmol}$ scale. After being heated to $60{ }^{\circ} \mathrm{C}$ for the appropriate time, the mixture was diluted with DCM, treated with $\mathrm{HCO}_{2} \mathrm{H}(0.1 \mathrm{~mL})$, and concentrated in vacuo. The yield was determined by ${ }^{1} \mathrm{H}$ NMR analysis of the crude product using $\mathrm{CH}_{2} \mathrm{Br}_{2}$ as the internal standard. The obtained yields were plotted as concentration vs. time (Figure S1 and S2). Representative initial data are shown below:

\begin{tabular}{cccc}
\hline entry & $\mathrm{t}(\mathrm{min})$ & $\mathbf{2 m}\left(10^{-3} \mathbf{M}\right)$ & $\mathbf{2 m}-\mathrm{d}_{4}\left(10^{-3} \mathbf{M}\right)$ \\
\hline 1 & 0 & 0.0 & 0.0 \\
2 & 20 & 22.5 & 19.3 \\
3 & 40 & 107.7 & 116.2 \\
4 & 60 & 161.0 & 141.3 \\
5 & 80 & 250.2 & 241.4 \\
\hline
\end{tabular}




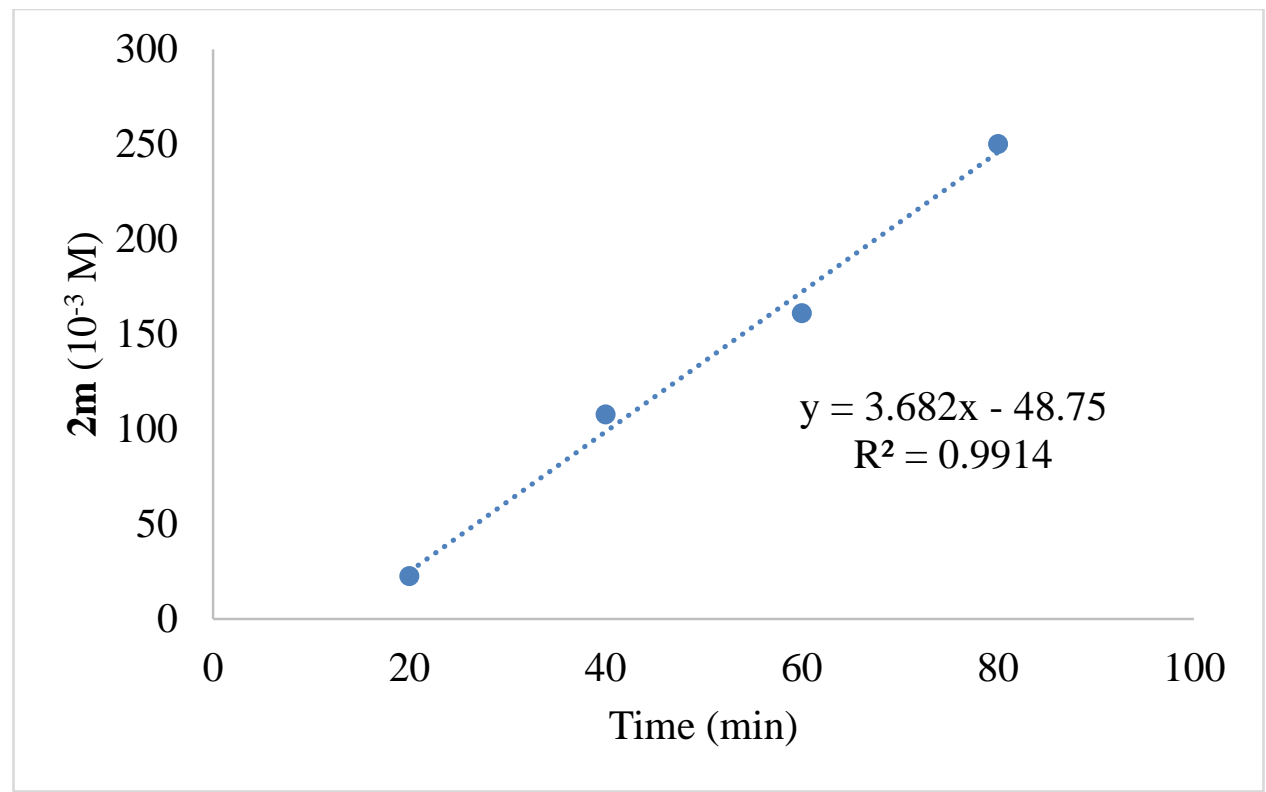

Figure S1. Representative initial data of $\mathbf{2 m}$

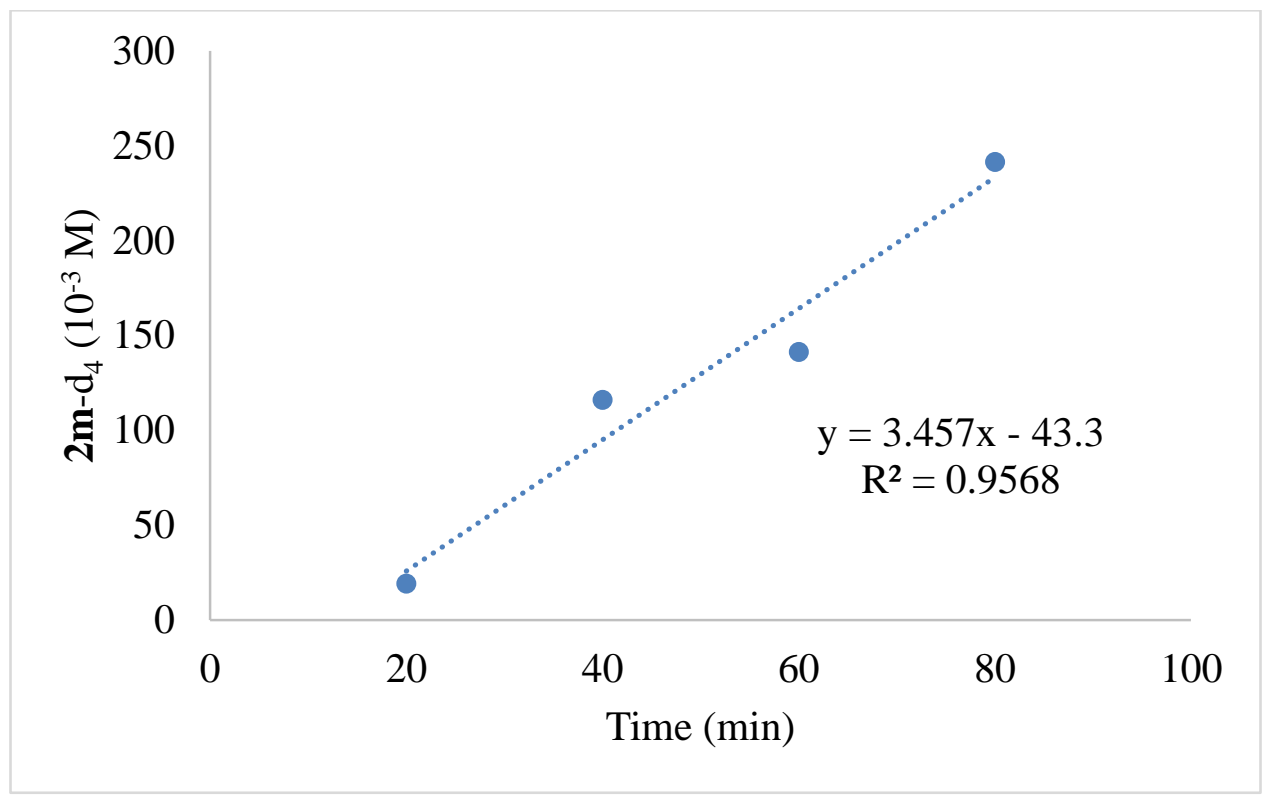

Figure S2. Representative initial data of $\mathbf{2 m}-\mathrm{d}_{4}$ 


\section{Reference:}

1. Park, H.; Chekshin, N.; Shen, P.-X.; Yu, J.-Q. Ligand-enabled, palladium-catalyzed $\beta$ $\mathrm{C}\left(\mathrm{sp}^{3}\right)-\mathrm{H}$ arylation of weinreb amides. ACS Catal. 2018, 8, 9292-9297.

2. Shen, P.-X.; Hu, L.; Shao, Q.; Hong, K.; Yu, J.-Q. Pd(II)-catalyzed enantioselective C( $\left.\mathrm{sp}^{3}\right)$ -H arylation of free carboxylic acids. J. Am. Chem. Soc. 2018, 140, 6545-6549.

3. Fillion, E.; Dumas, A. M. Synthesis of fused 4,5-disubstituted indole ring systems by intramolecular Friedel-Crafts acylation of 4-substituted indoles. J. Org. Chem. 2008, 73, $2920-2923$.

4. Franchini, C.; Carocci, A.; Catalano, A.; Cavalluzzi, M. M.; Corbo, F.; Lentini, G.; Scilimati, A.; Tortorella, P.; Camerino, D. C.; De Luca, A. J. Optically active mexiletine analogues as stereoselective blockers of voltage-gated $\mathrm{Na}^{+}$channels. J. Med. Chem. 2003, $46,5238-5248$.

5. Ikeda, K.; Achiwa, K.; Sekiya, M. Trifluoromethanesulfonic acid-promoted reaction of hexahydro-1,3,5-triazines. Introduction of a secondary aminomethyl grouping into carboxylates at the $\alpha$-position through ketene silyl acetals. Chem. Pharm. Bull. 1986, 34, $1579-1583$.

6. Hong, K.; Park, H.; Yu, J.-Q. Methylene $\mathrm{C}\left(\mathrm{sp}^{3}\right)-\mathrm{H}$ arylation of aliphatic ketones using a transient directing group. ACS Catal. 2017, 7, 6938-6941.

7. Naturale, G.; Lamblin, M.; Commandeur, C.; Felpin, F.-X.; Dessolin, J. Direct C-H alkylation of naphthoquinones with amino acids through a revisited Kochi-Anderson radical decarboxylation: trends in reactivity and applications. Eur. J. Org. Chem. 2012, 5774-5788.

8. Dener, J. M.; Fantauzzi, P. P.; Kshirsagar, T. A.; Kelly, D. E.; Wolfe, A. B. Large-scale syntheses of FMOC-protected non-proteogenic amino acids: useful building blocks for combinatorial libraries. Org. Process Res. Dev. 2001, 54, 445-449.

9. F. Fülöp, M. Palkó, J. Kámán, L. Lázár, R. Sillanpää, Synthesis of all four enantiomers of 1-aminoindane-2-carboxylic acid, a new cispentacin benzologue. Tetrahedron: Asymmetry 2000, 11, 4179-4187.

10. Stavber, S.; Kralj, P.; Zupan, M. Selective and effective iodination of alkyl-substituted benzenes with elemental iodine activated by Selectfluor ${ }^{\mathrm{TM}}$ F-TEDA-BF 4 . Synlett 2002, 598-600. 
11. Seo, H.; Liu, A.; Jamison, T. F. Direct $\beta$-selective hydrocarboxylation of styrenes with $\mathrm{CO}_{2}$ enabled by continuous flow photoredox catalysis. J. Am. Chem. Soc. 2017, 139, 13969-13972.

12. Feng, Y. et al. Benzopyrans and analogs as Rho kinase inhibitors and their preparation and use in the treatment of Rho kinase-mediated diseases. PCT Int. Appl., 2009079008, 25 Jun 2009.

13. Alkayal, A.; Tabas, V.; Montanaro, S.; Wright, I. A.; Malkov, A. V.; Buckley, B. R. Harnessing applied potential: selective $\beta$-hydrocarboxylation of substituted olefins. $J$. Am. Chem. Soc. 2020, 142, 1780-1785.

14. Melot, R.; Craveiro, M.; Bürgi, T.; Baudoin, O. Divergent enantioselective synthesis of (nor)illudalane sesquiterpenes via $\mathrm{Pd}^{0}$-catalyzed asymmetric $\mathrm{C}\left(\mathrm{sp}^{3}\right)-\mathrm{H}$ activation. Org. Lett. 2019, 21, 812-815.

15. Melot, R.; Craveiro, M. V.; Baudoin, O. Total synthesis of (nor)illudalane sesquiterpenes based on a $\mathrm{C}\left(\mathrm{sp}^{3}\right)-\mathrm{H}$ activation strategy. J. Org. Chem. 2019, 84, 12933-12945. 


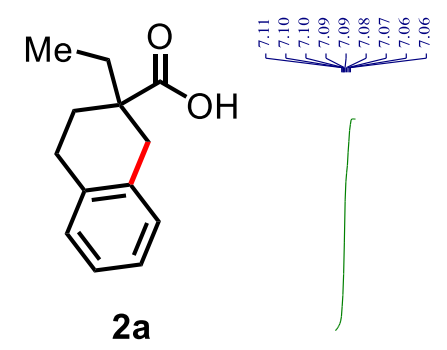

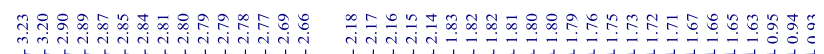
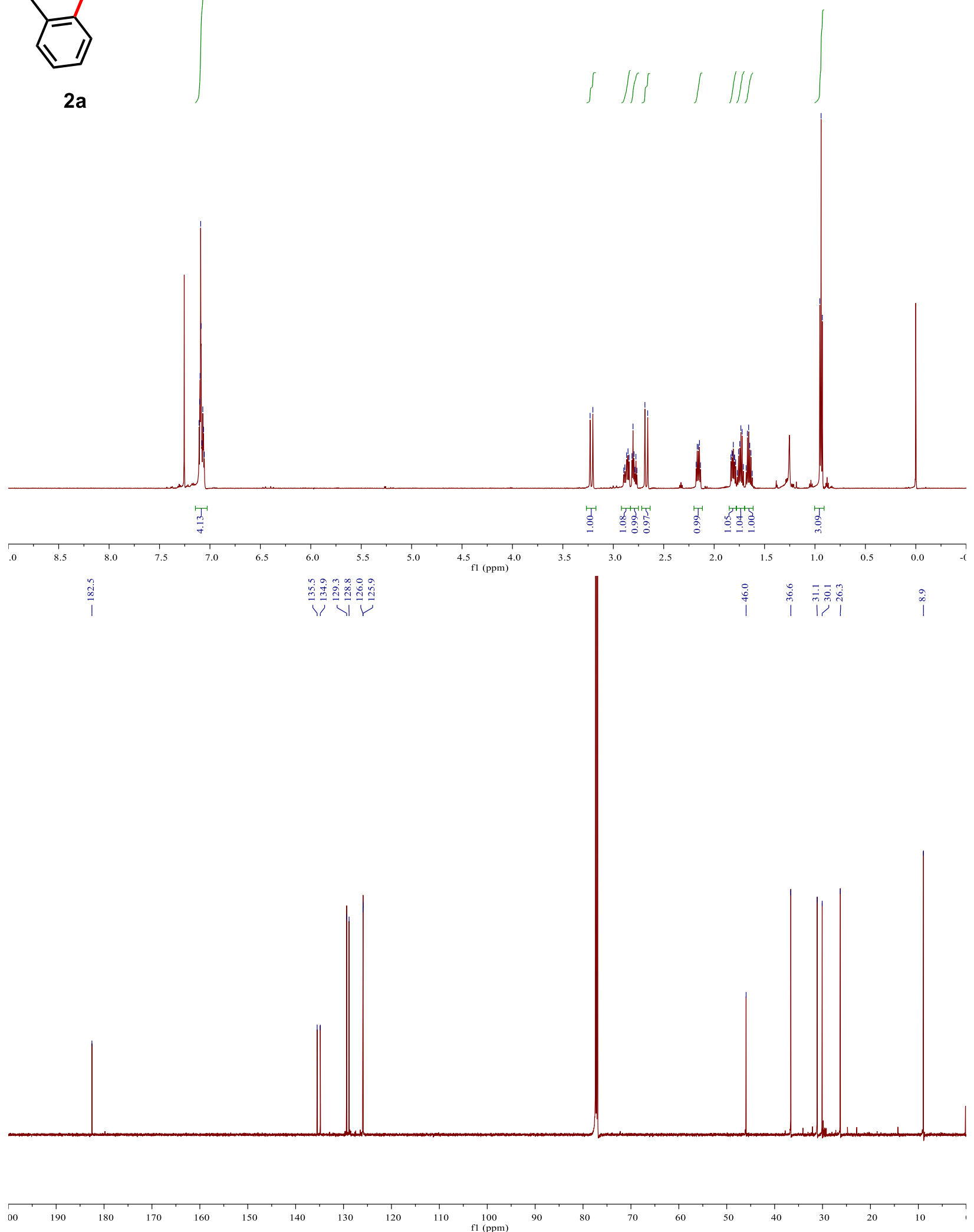


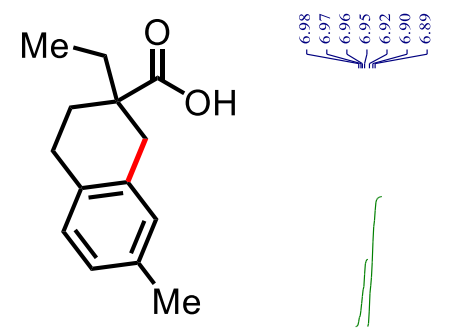

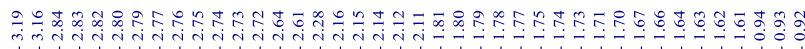

$2 b$
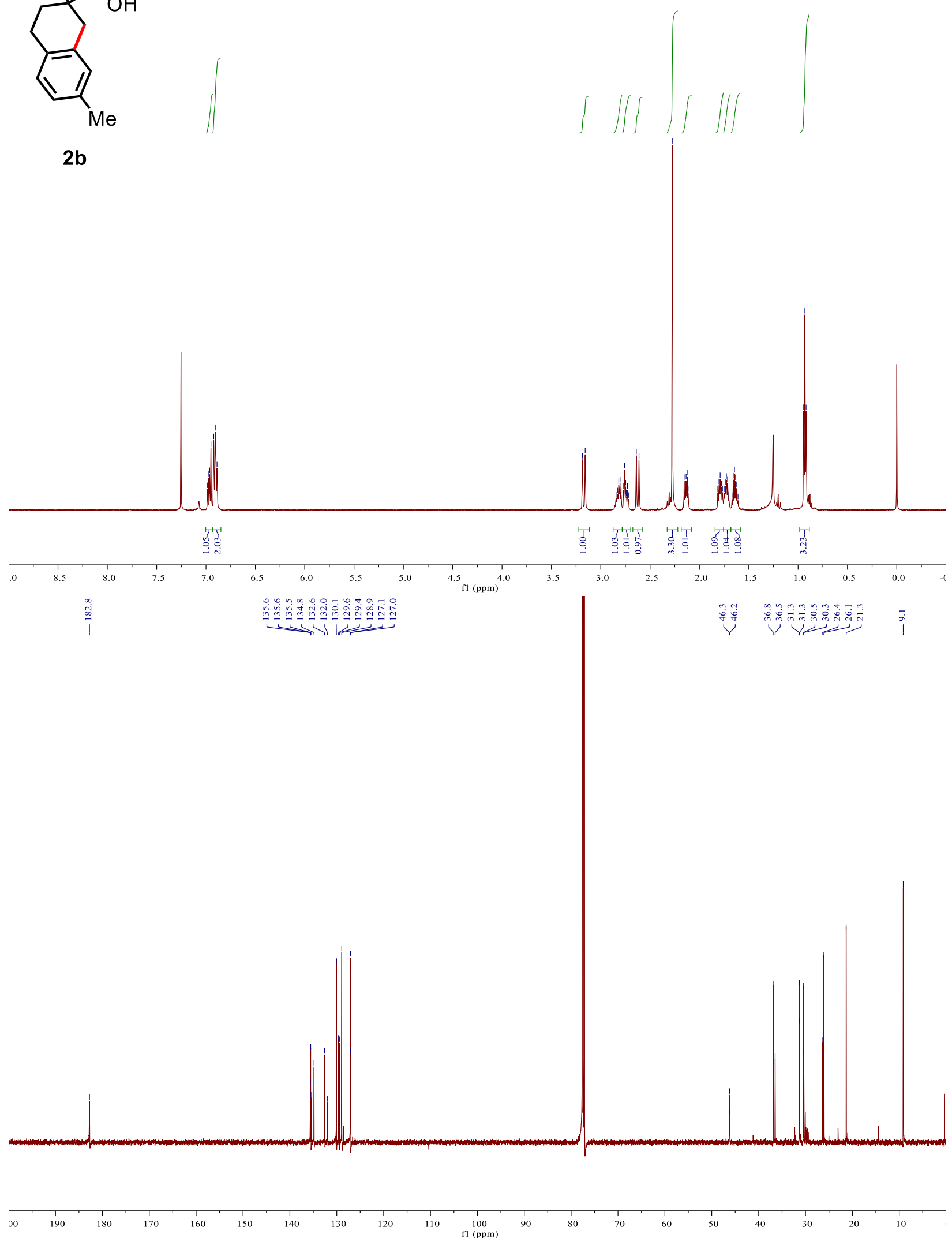


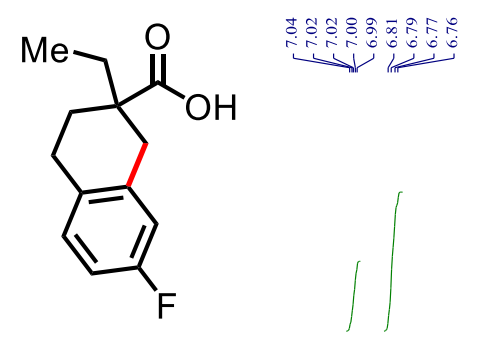

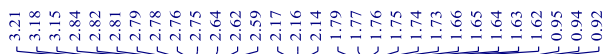

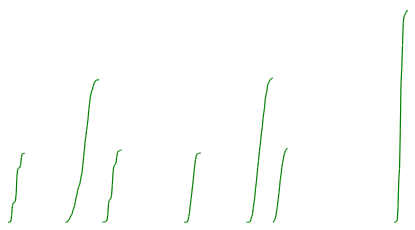

2c

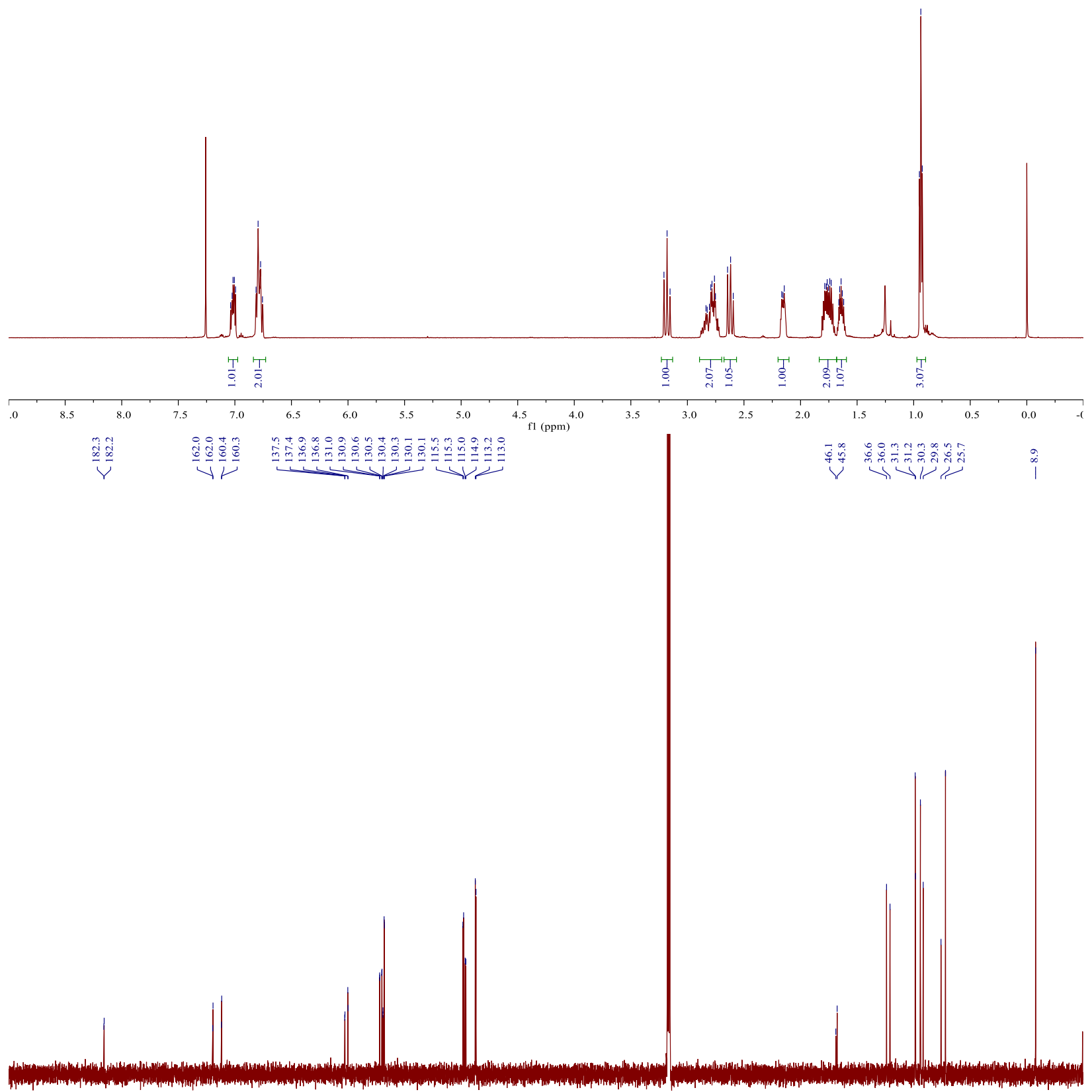

00

(1.0. 


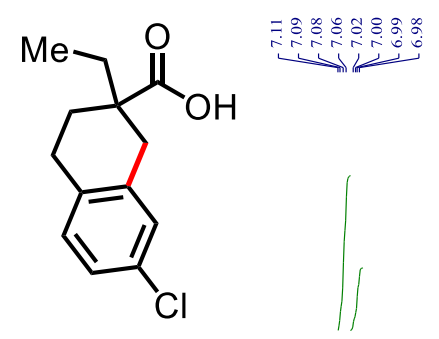

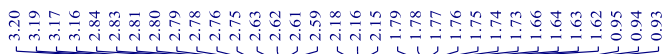

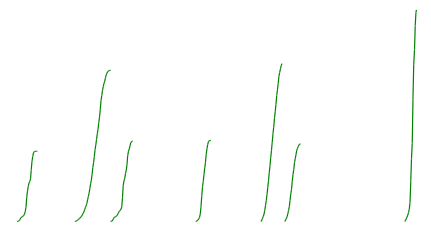

2d

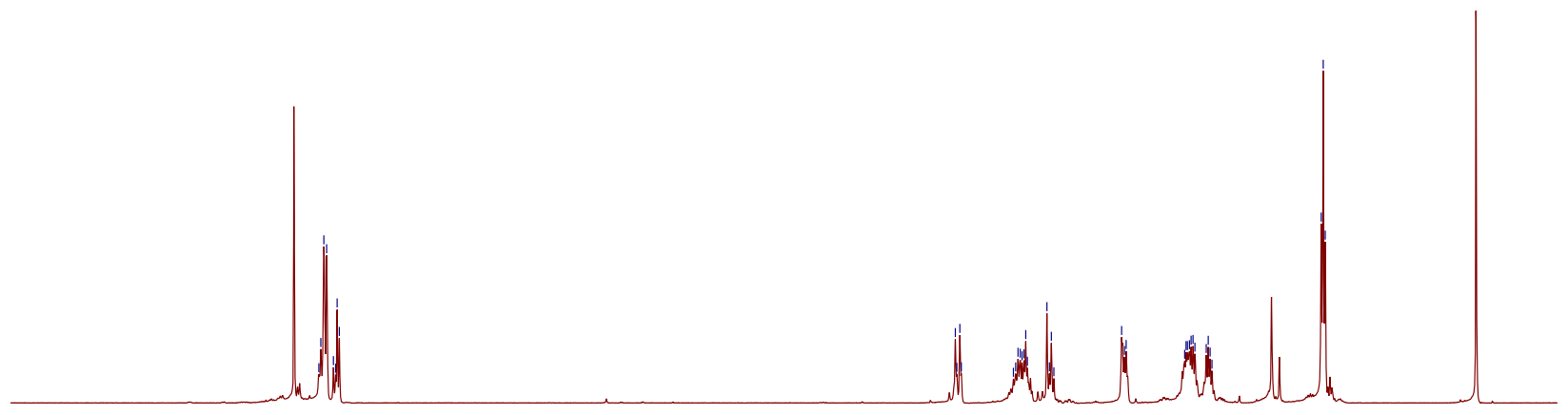

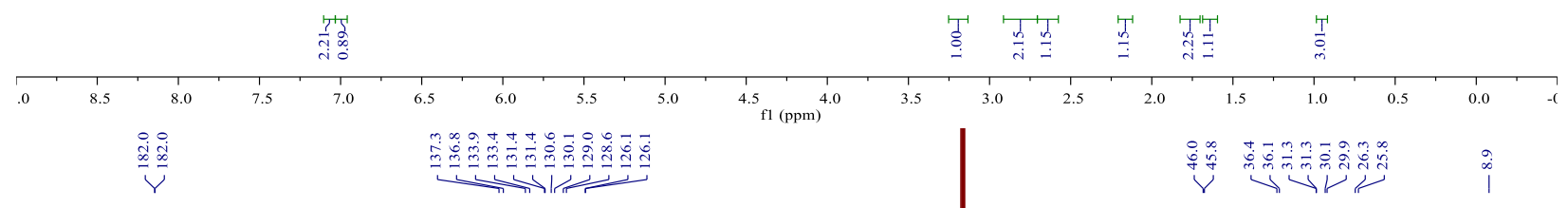

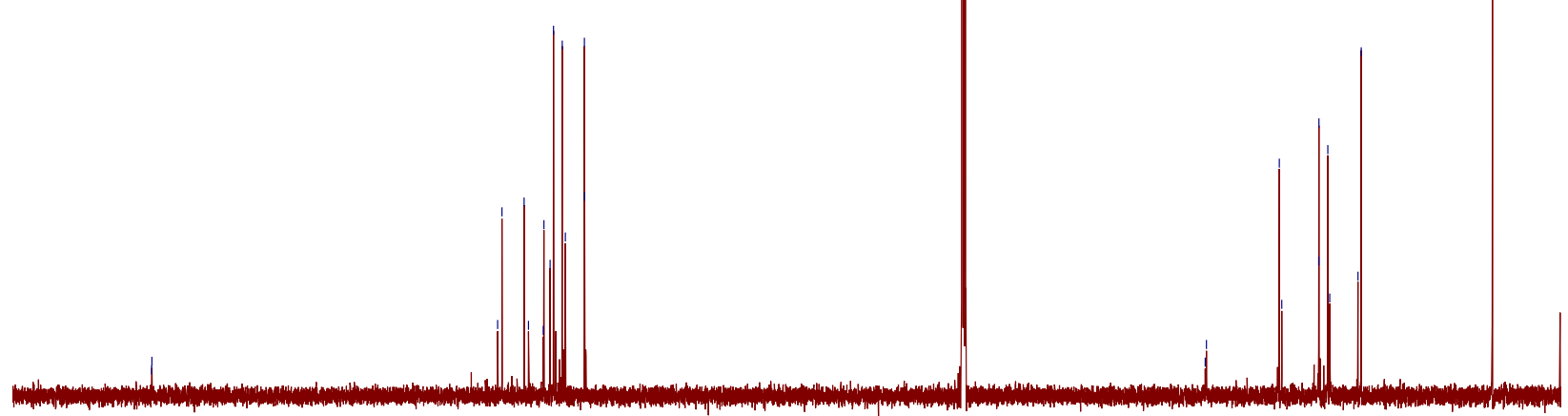

00
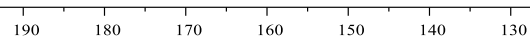

100 


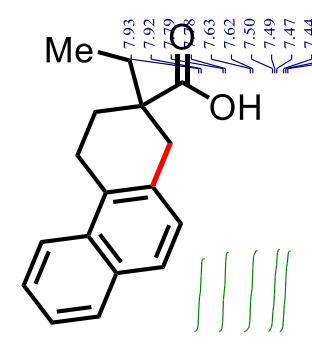

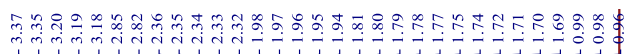

$2 e$
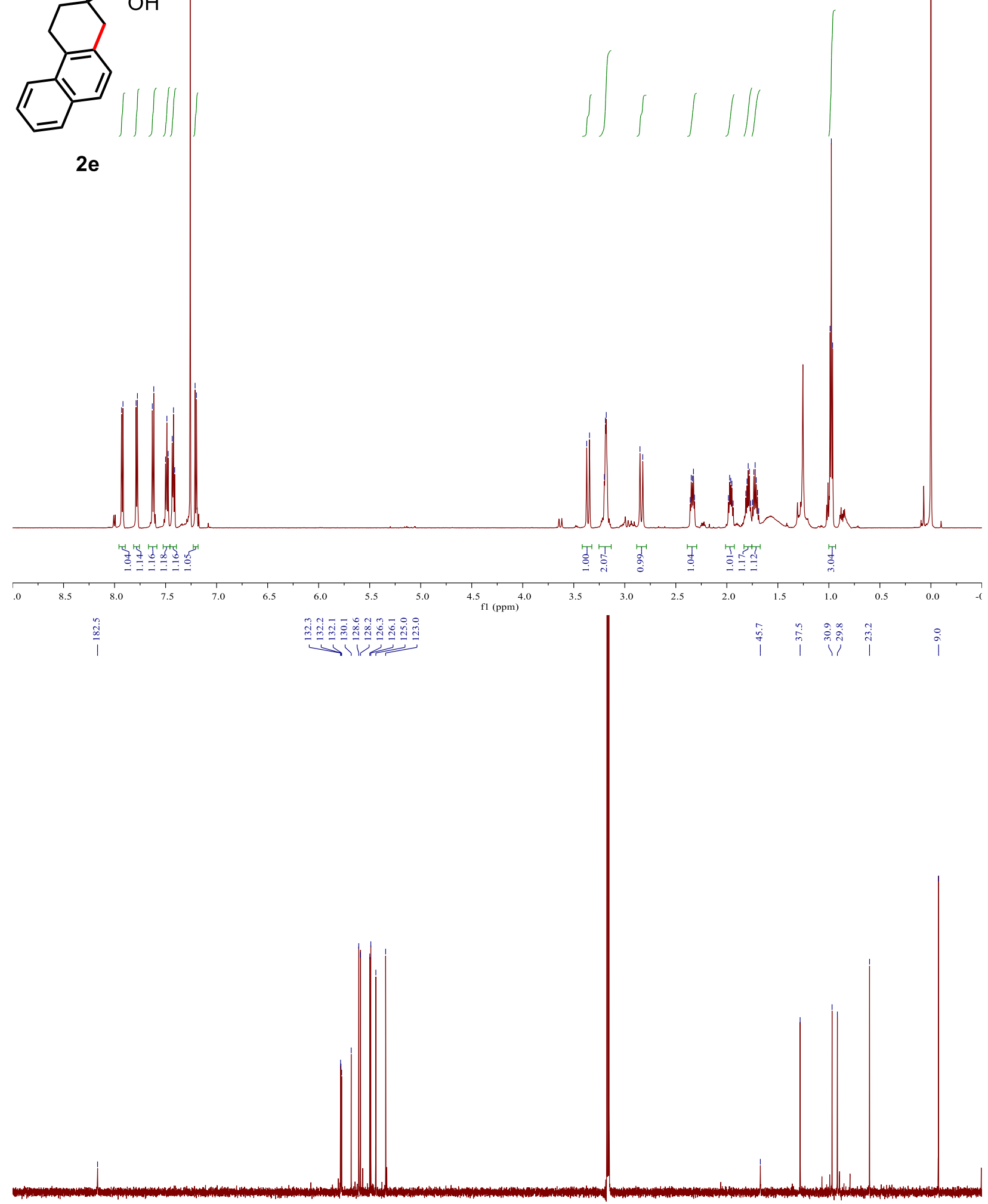

00
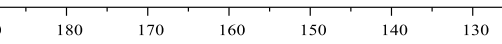

100
fl $(\mathrm{ppm})$ 

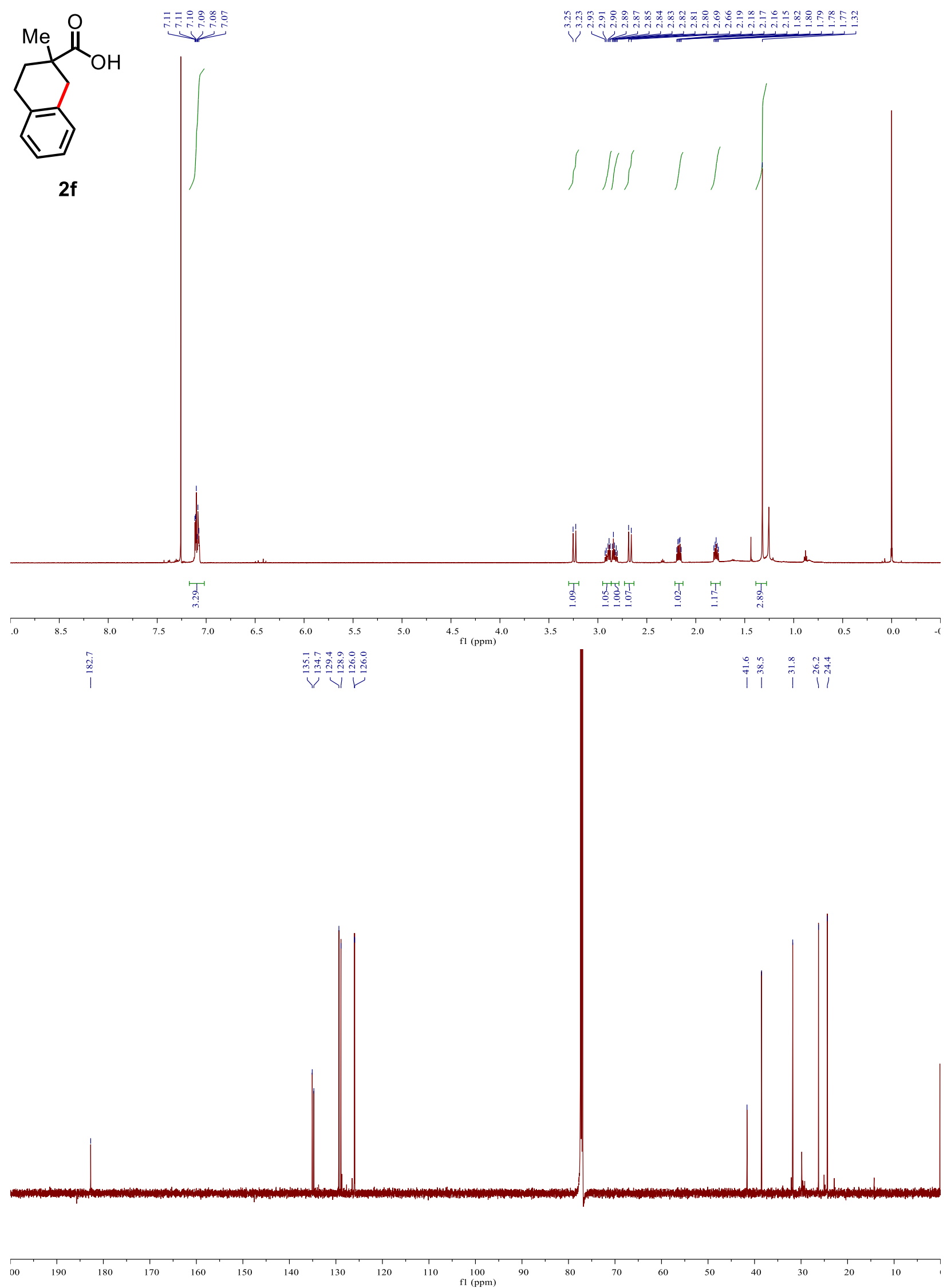


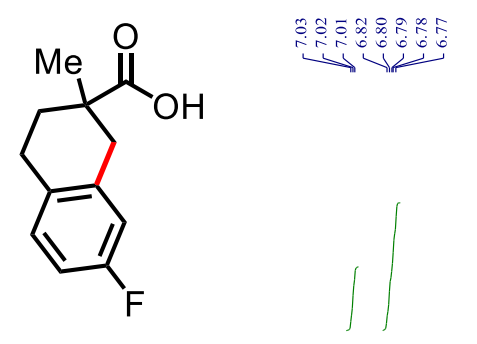

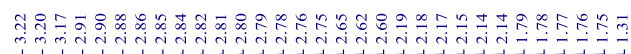

2g
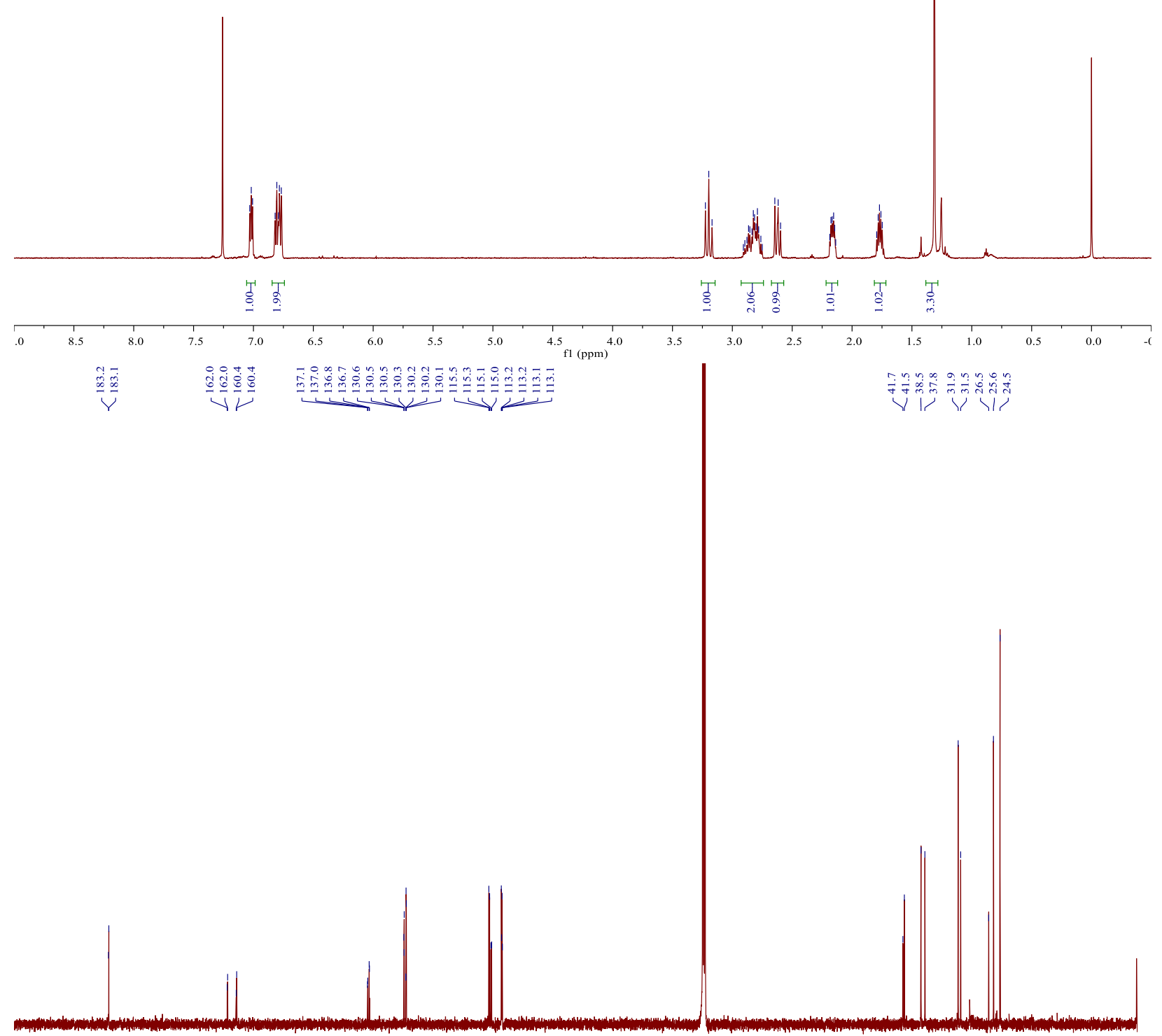

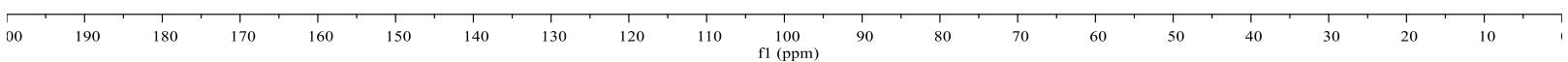



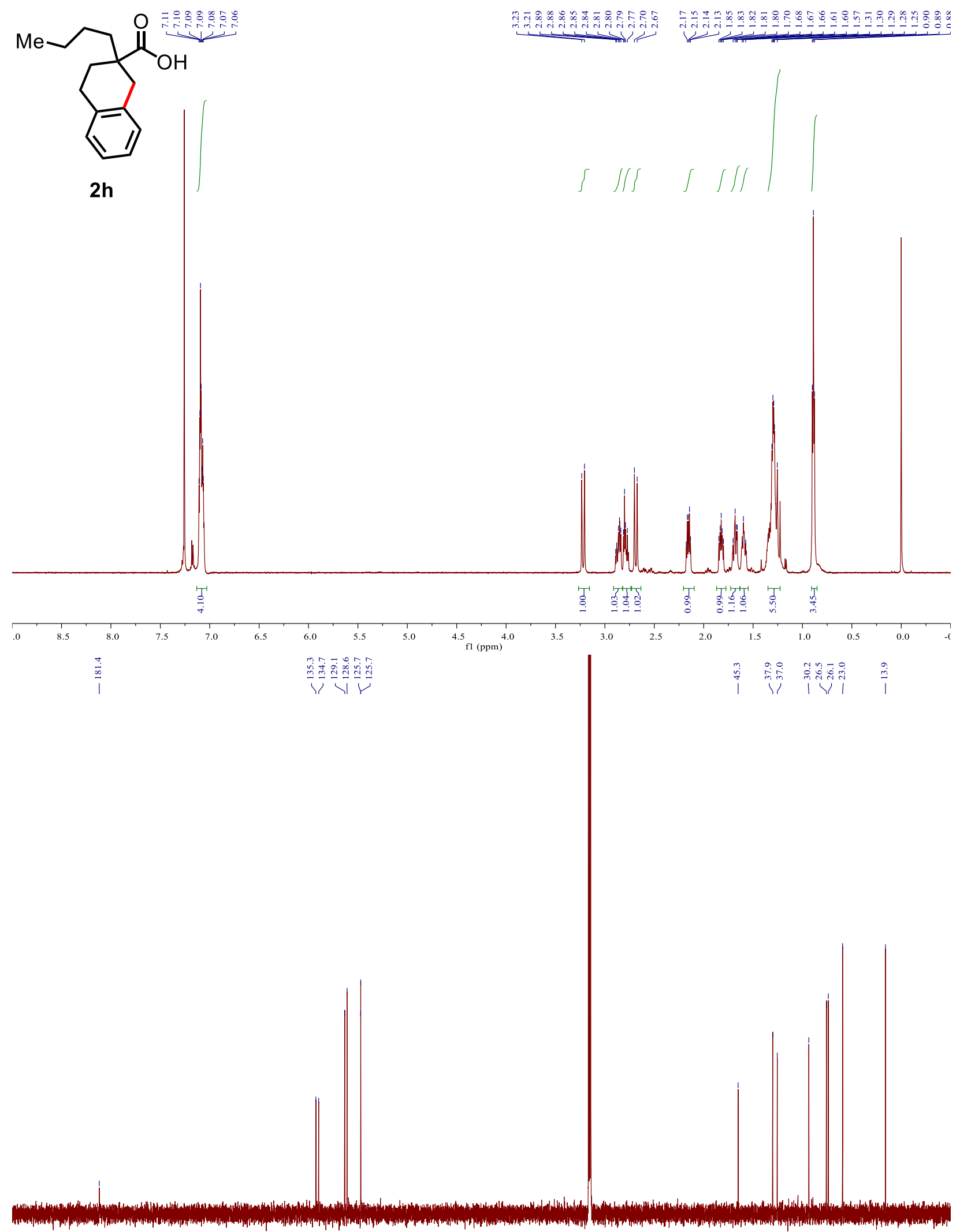

00
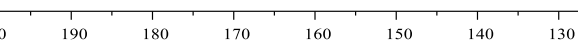

100
fl $(\mathrm{ppm})$ 

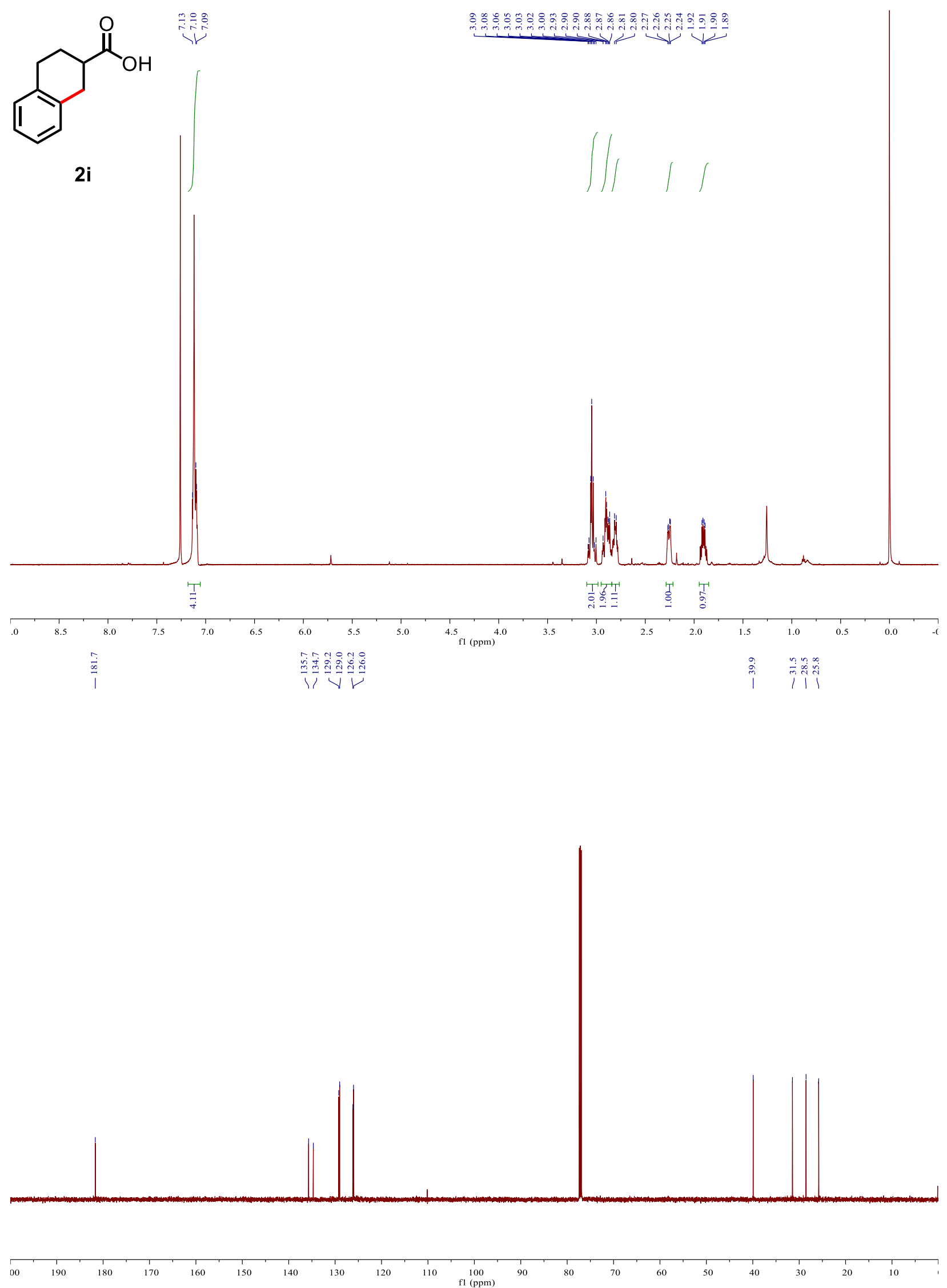


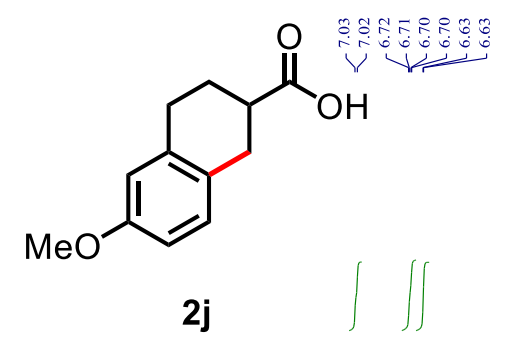

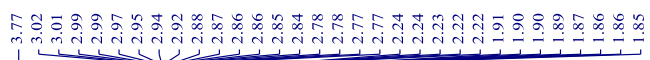
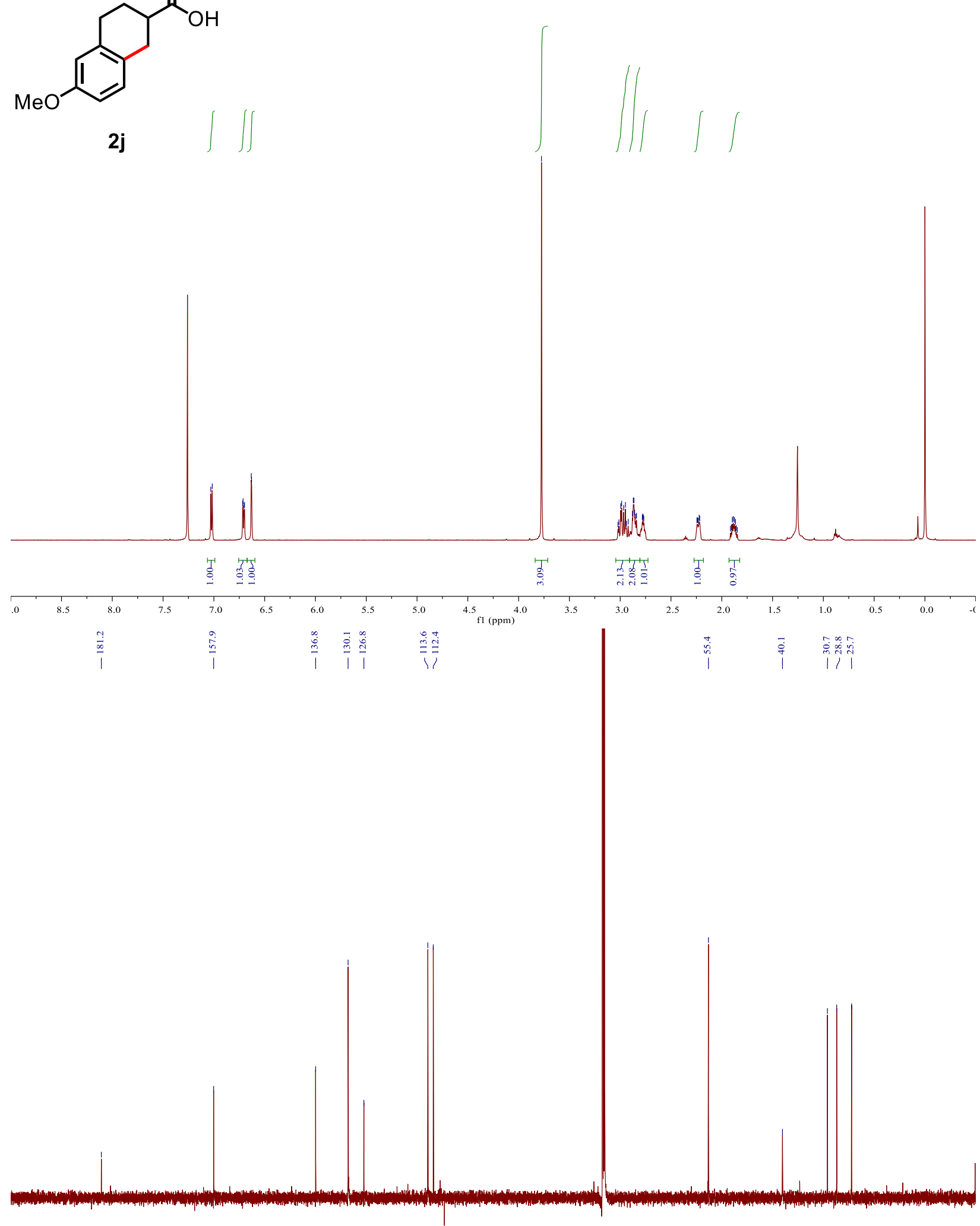

00
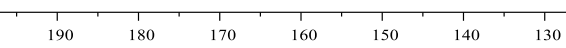

100 

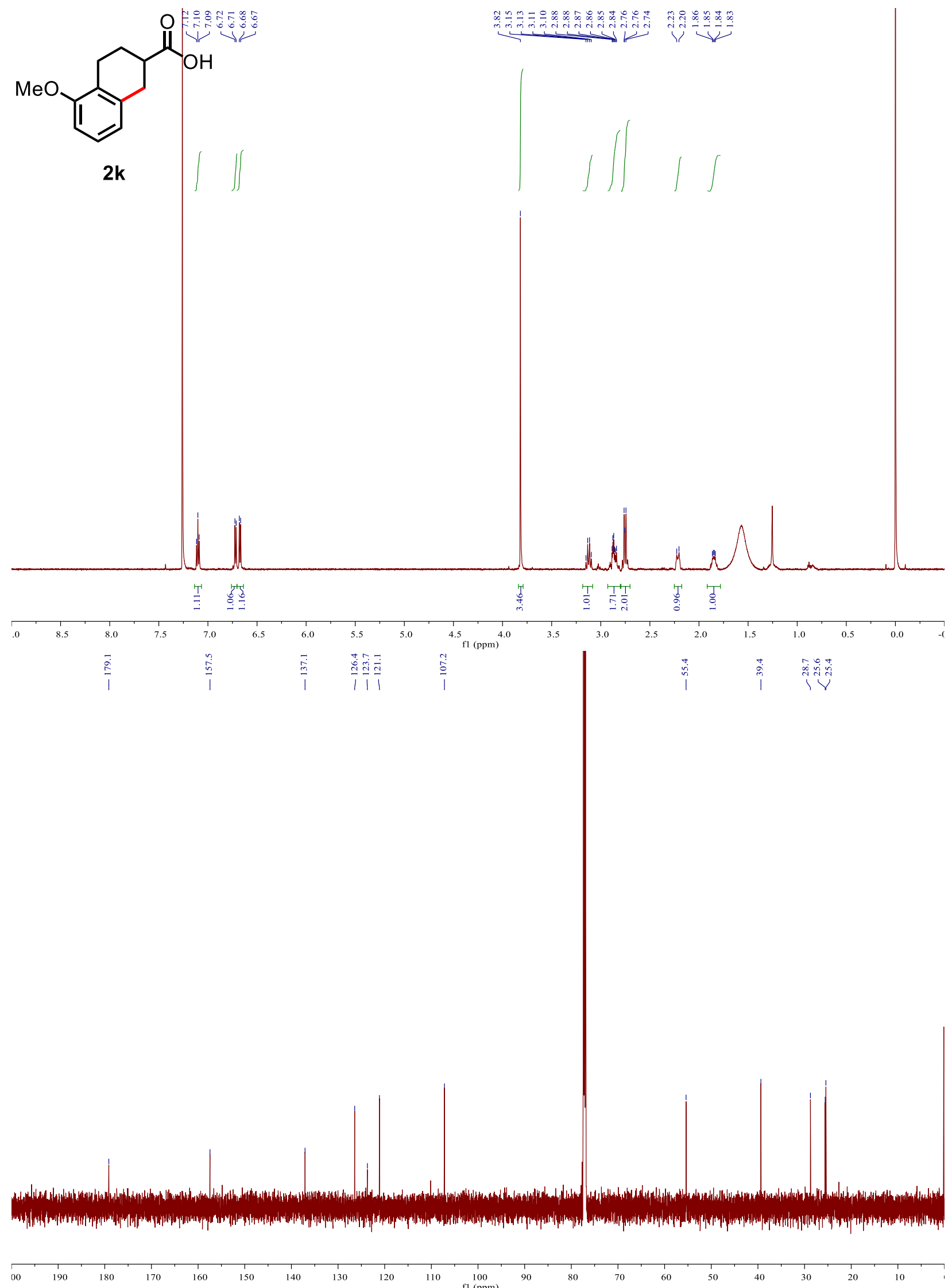

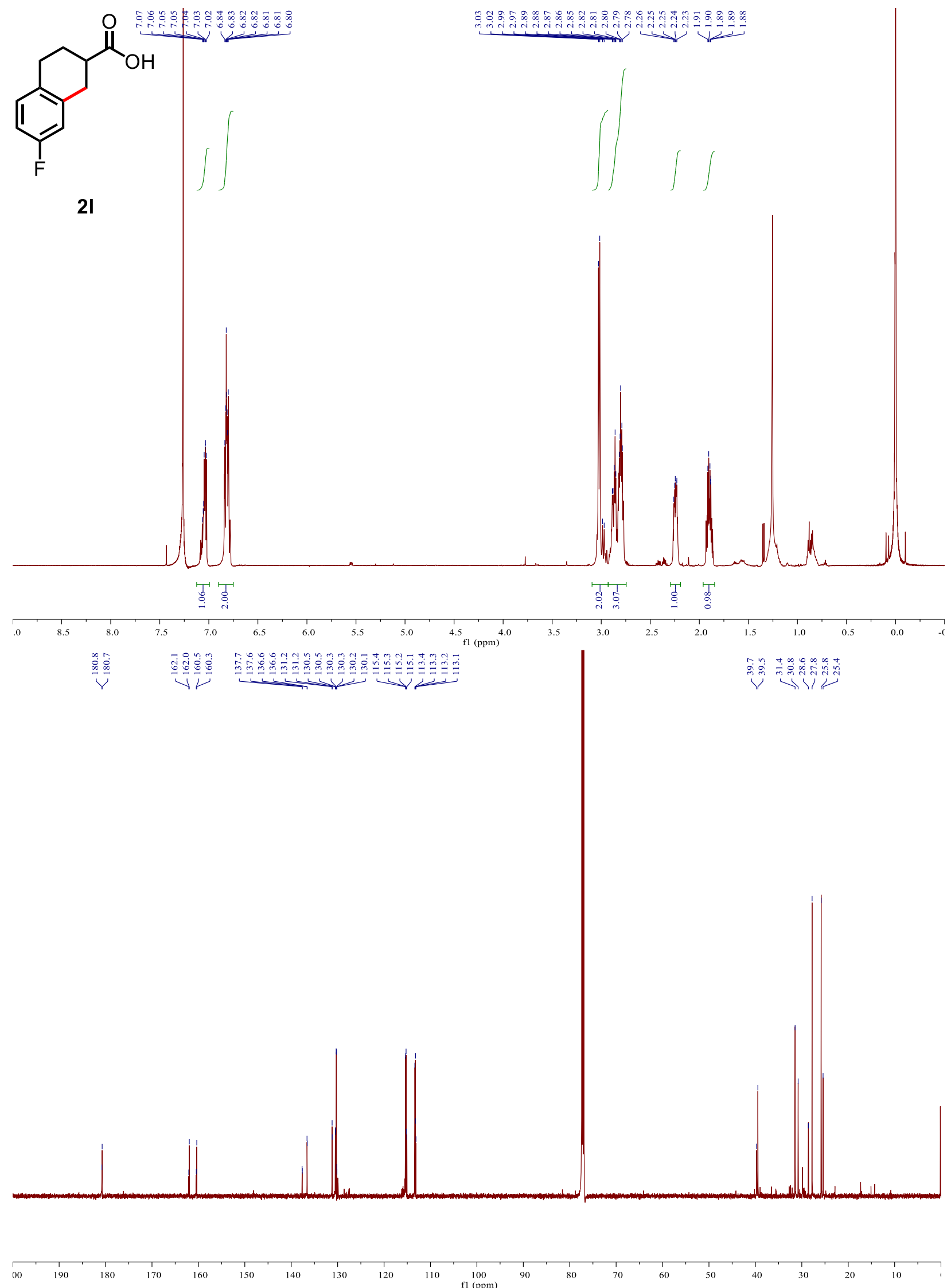

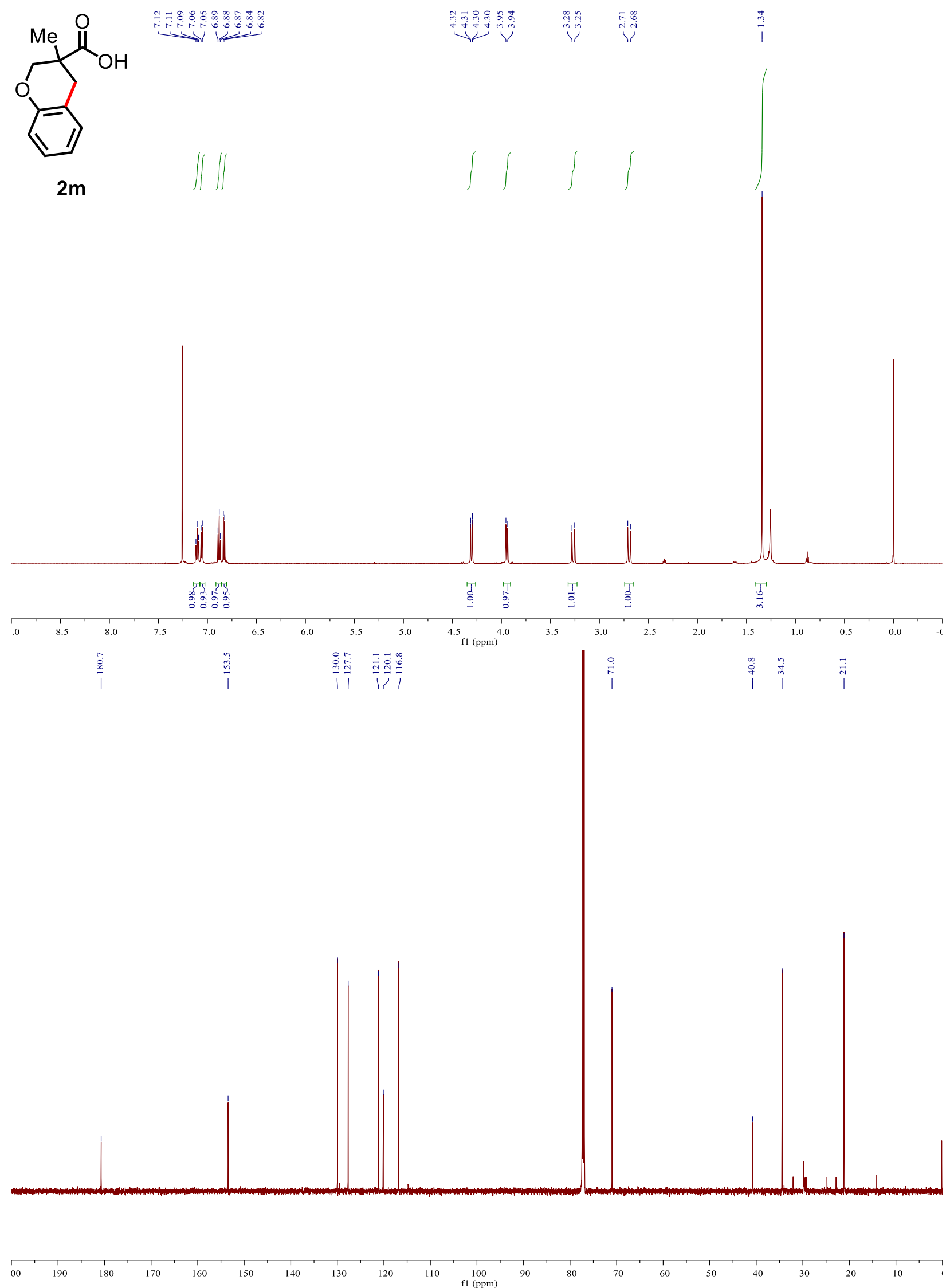


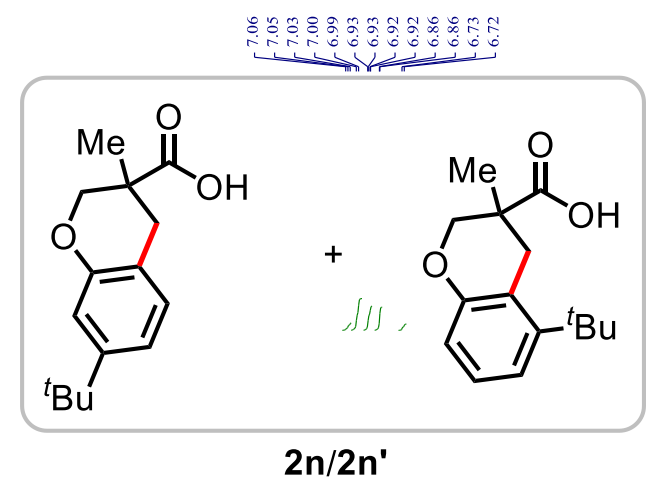

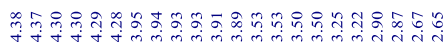

$2 n / 2 n$
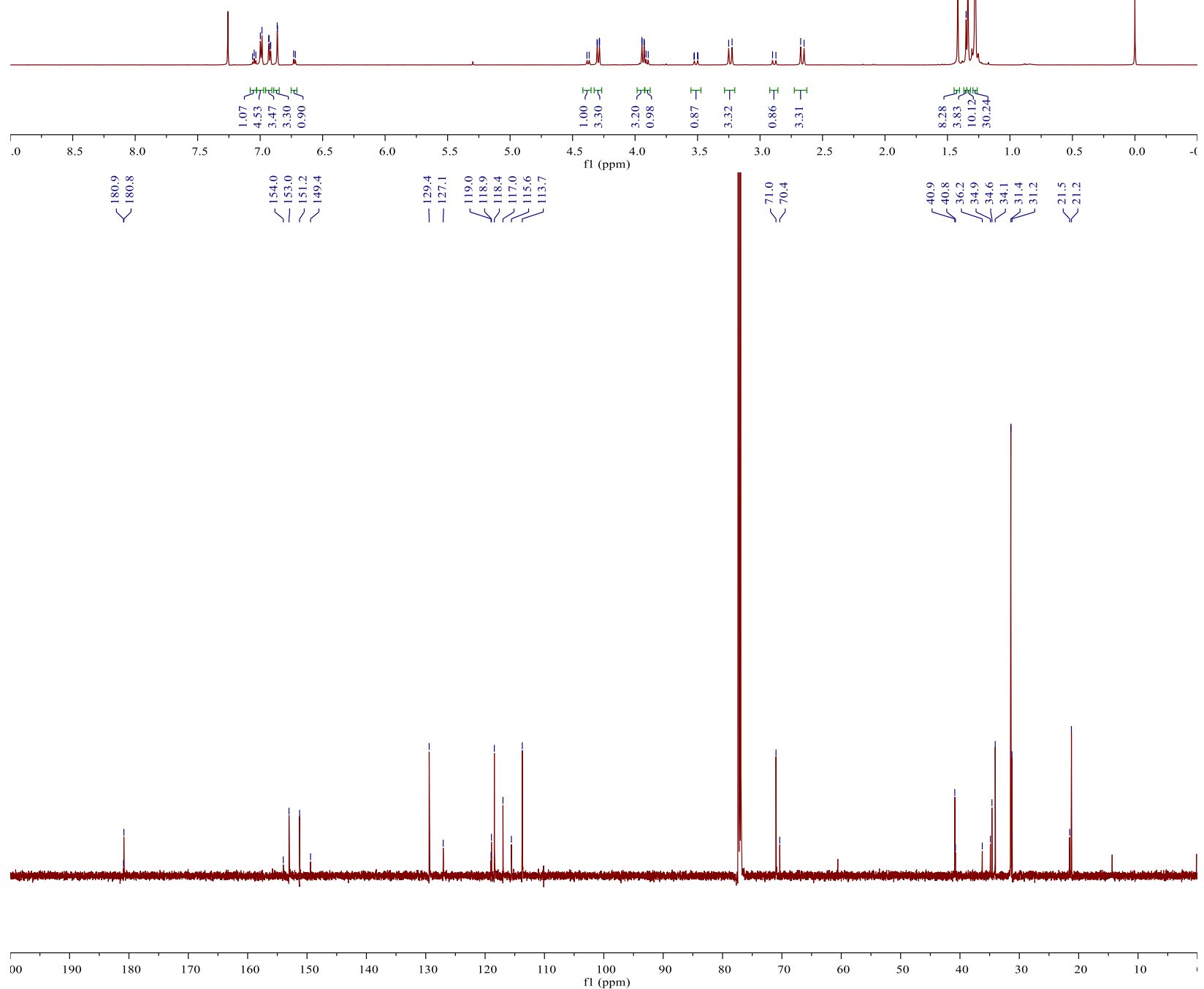


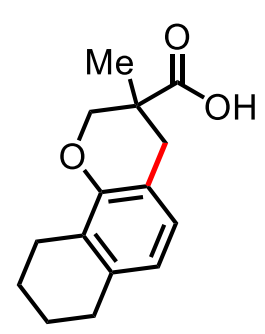

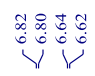

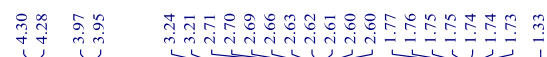

20
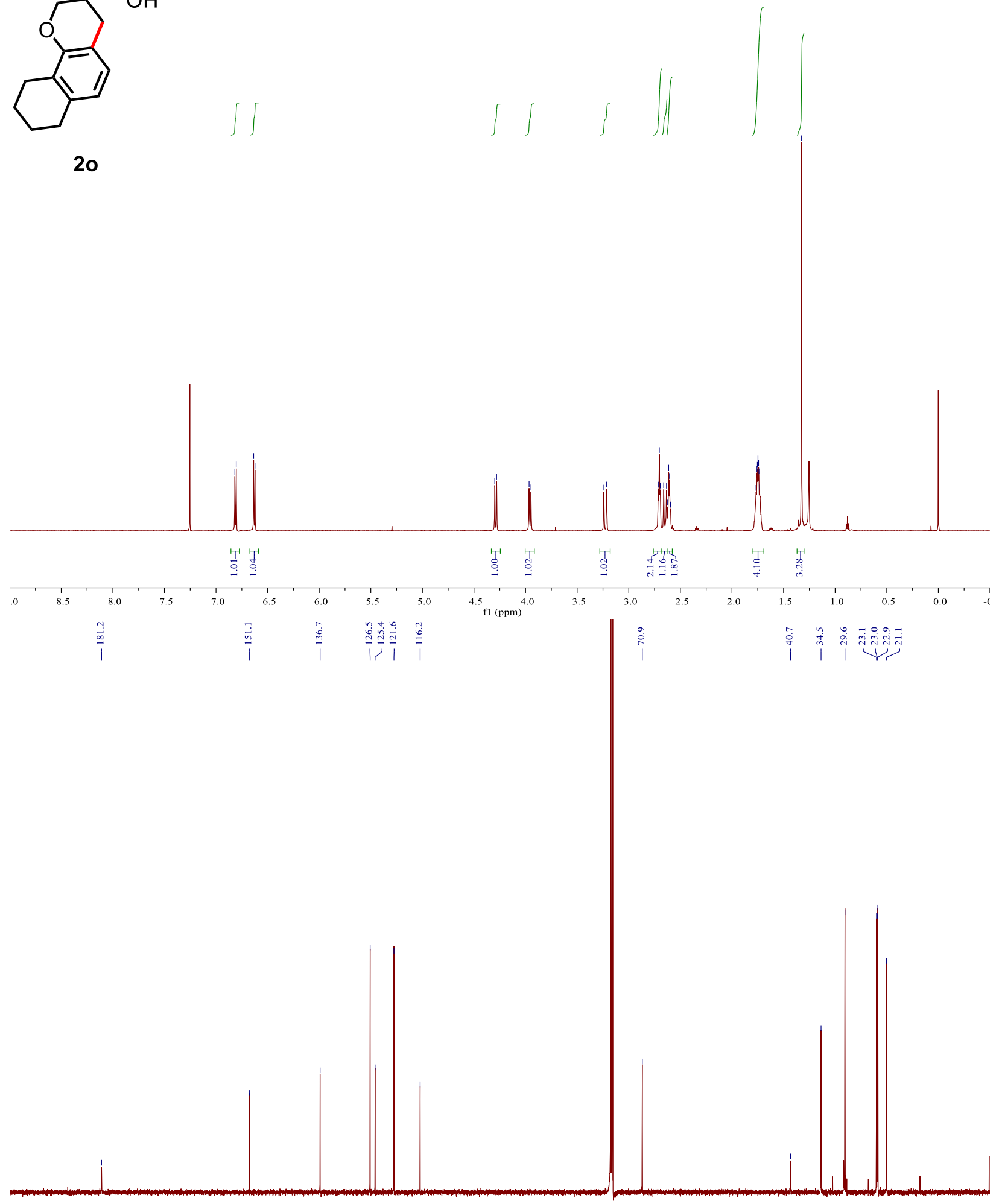

00
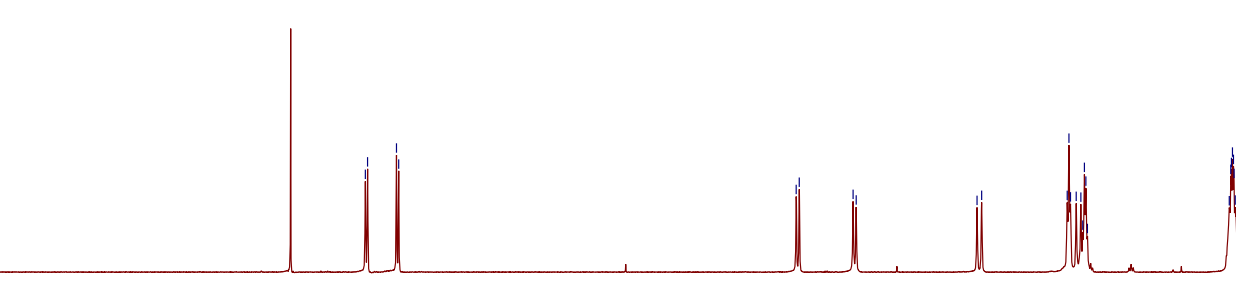

I I V
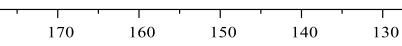

100 

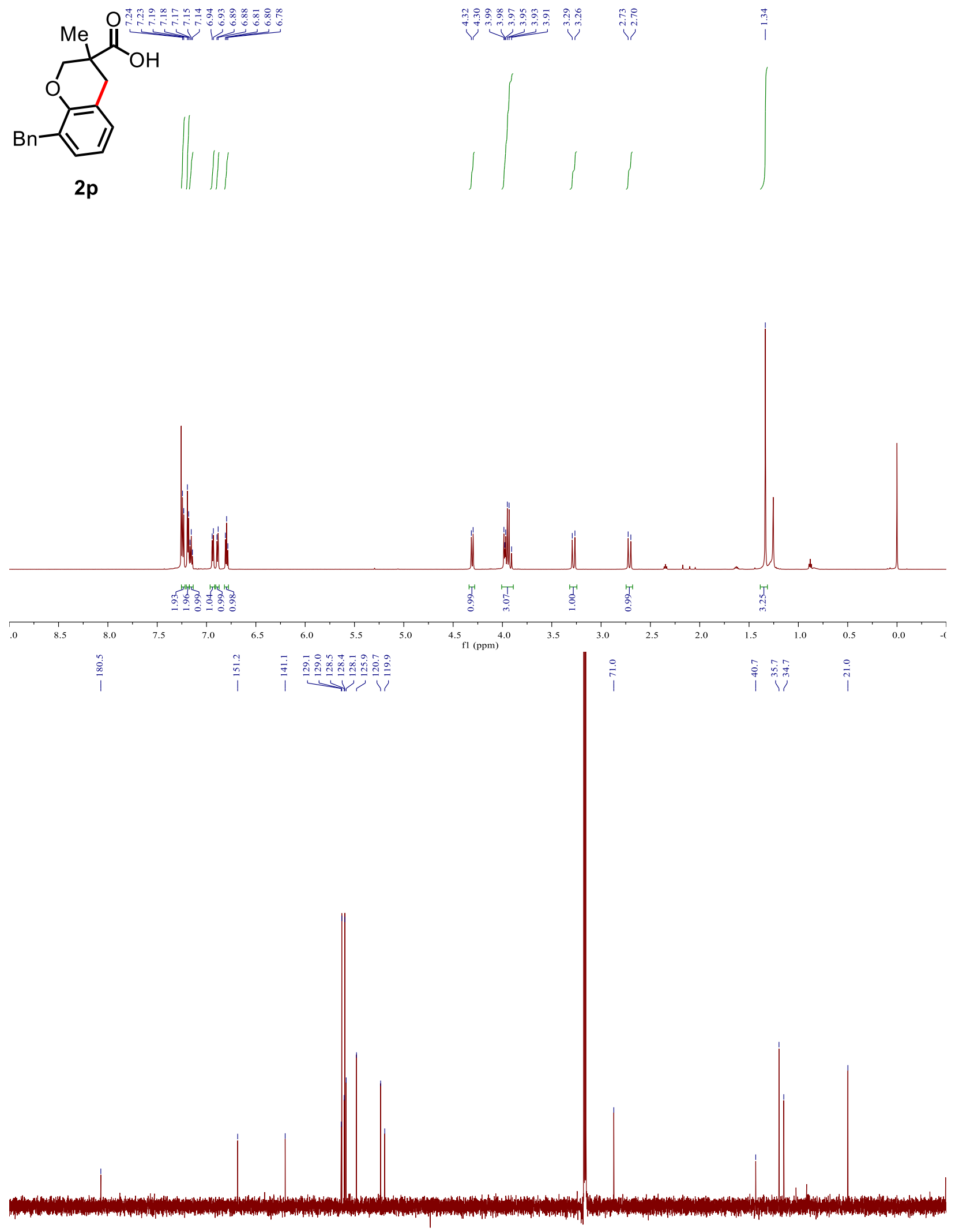

00
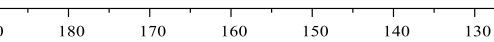

100 


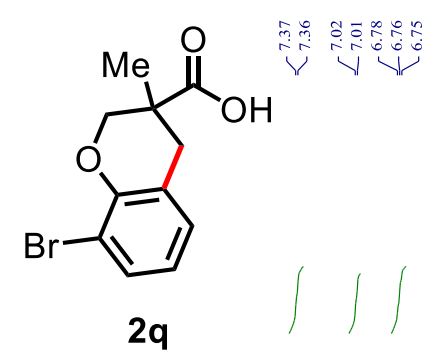

Vij
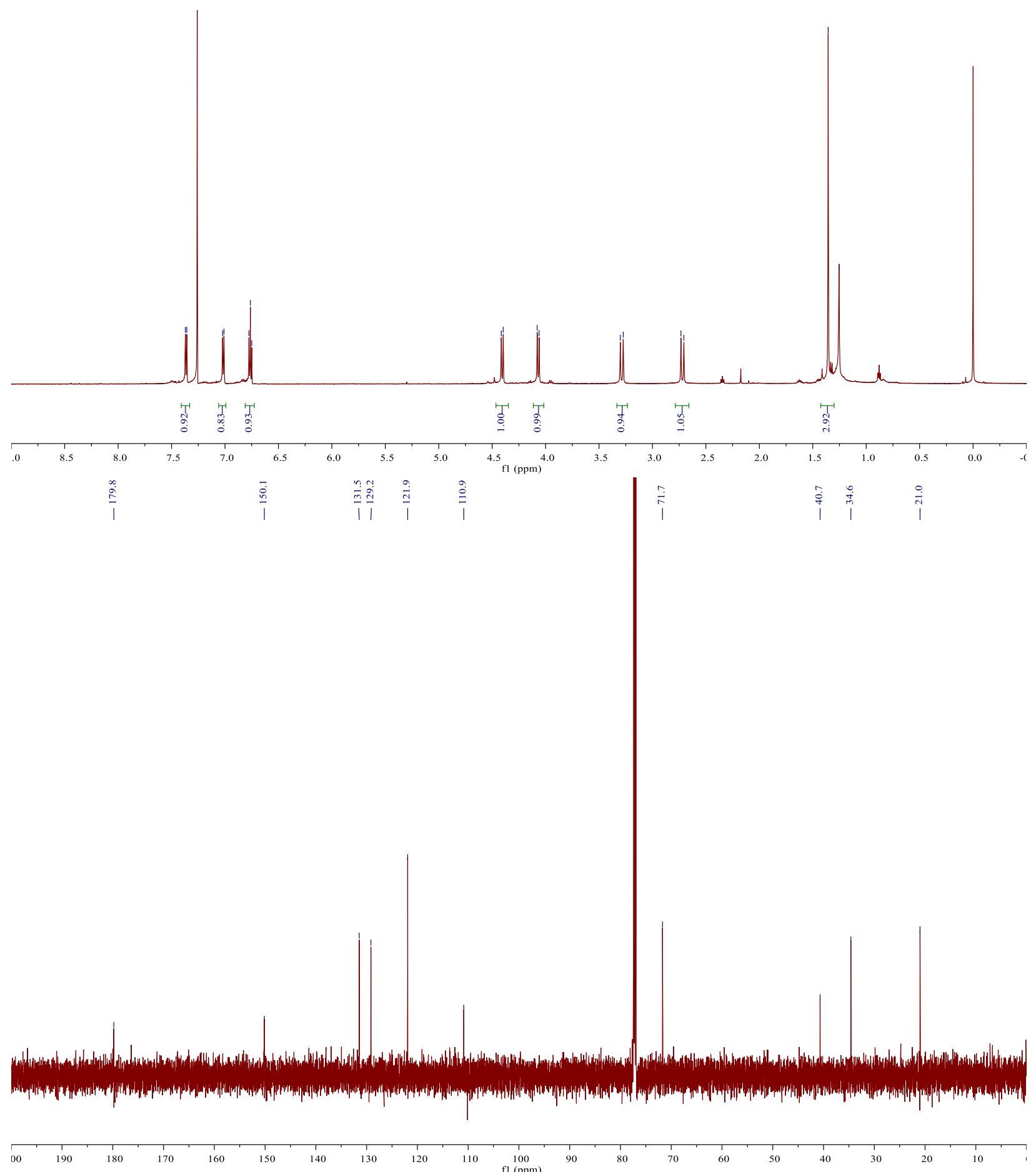

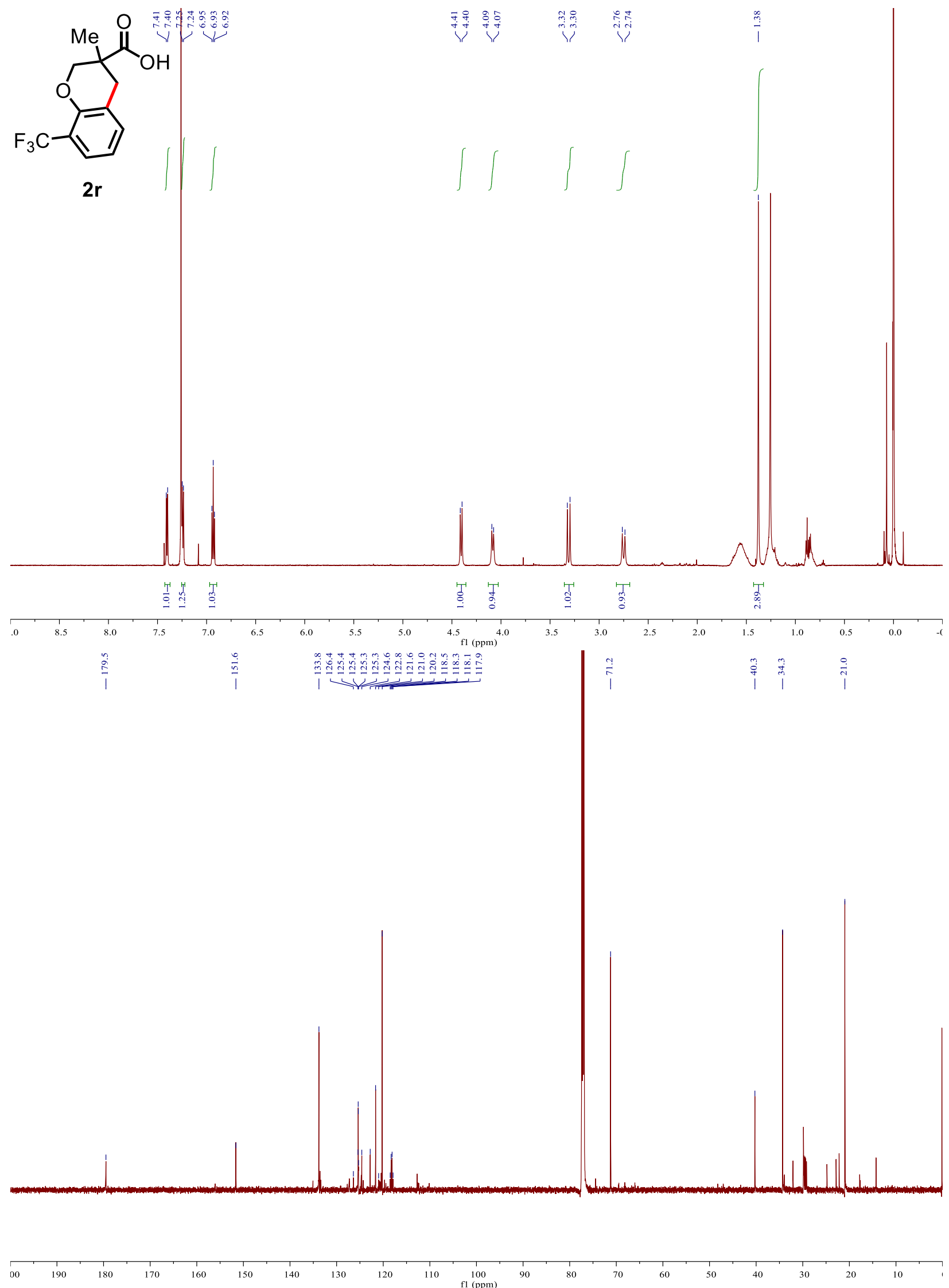

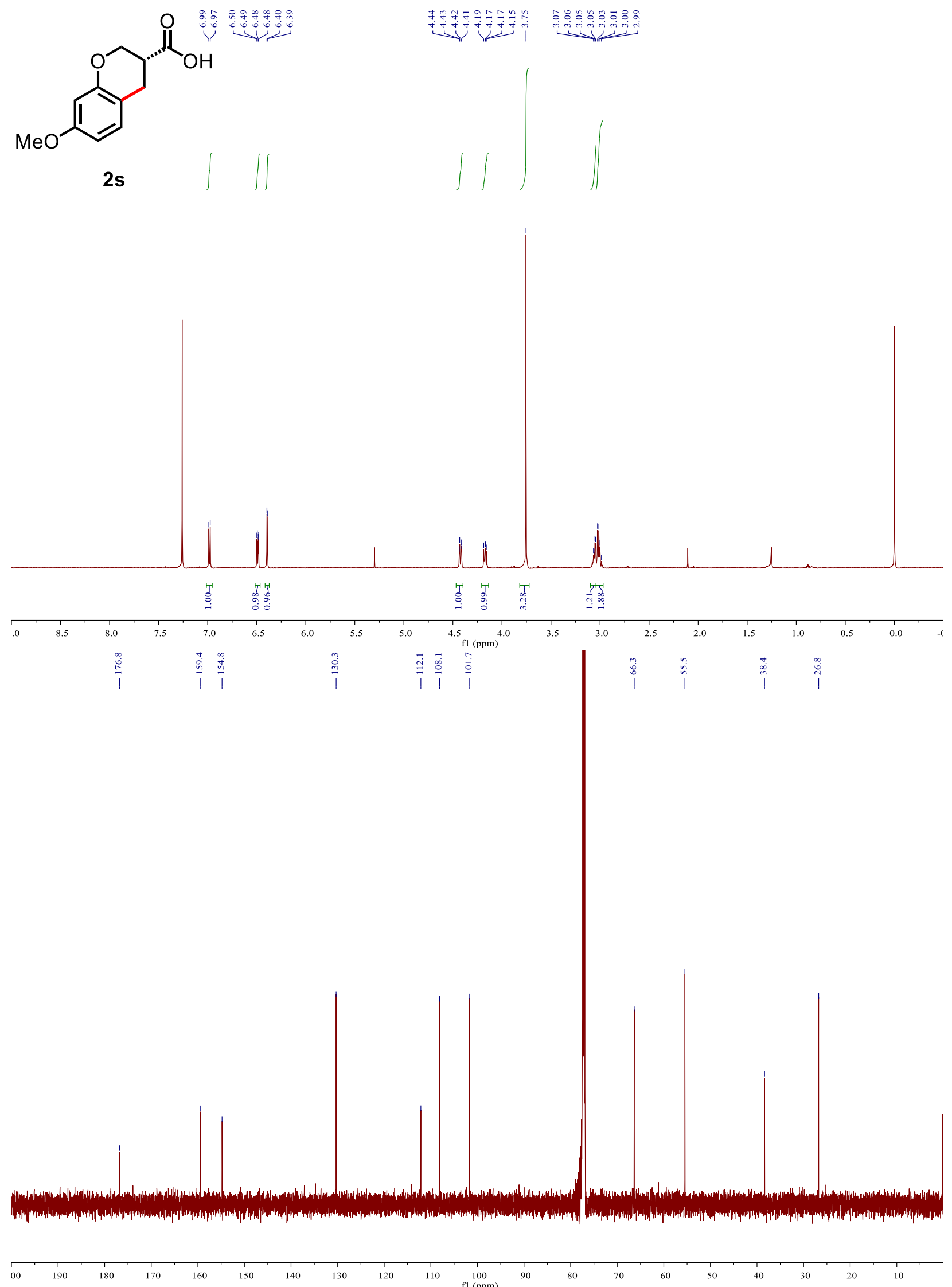

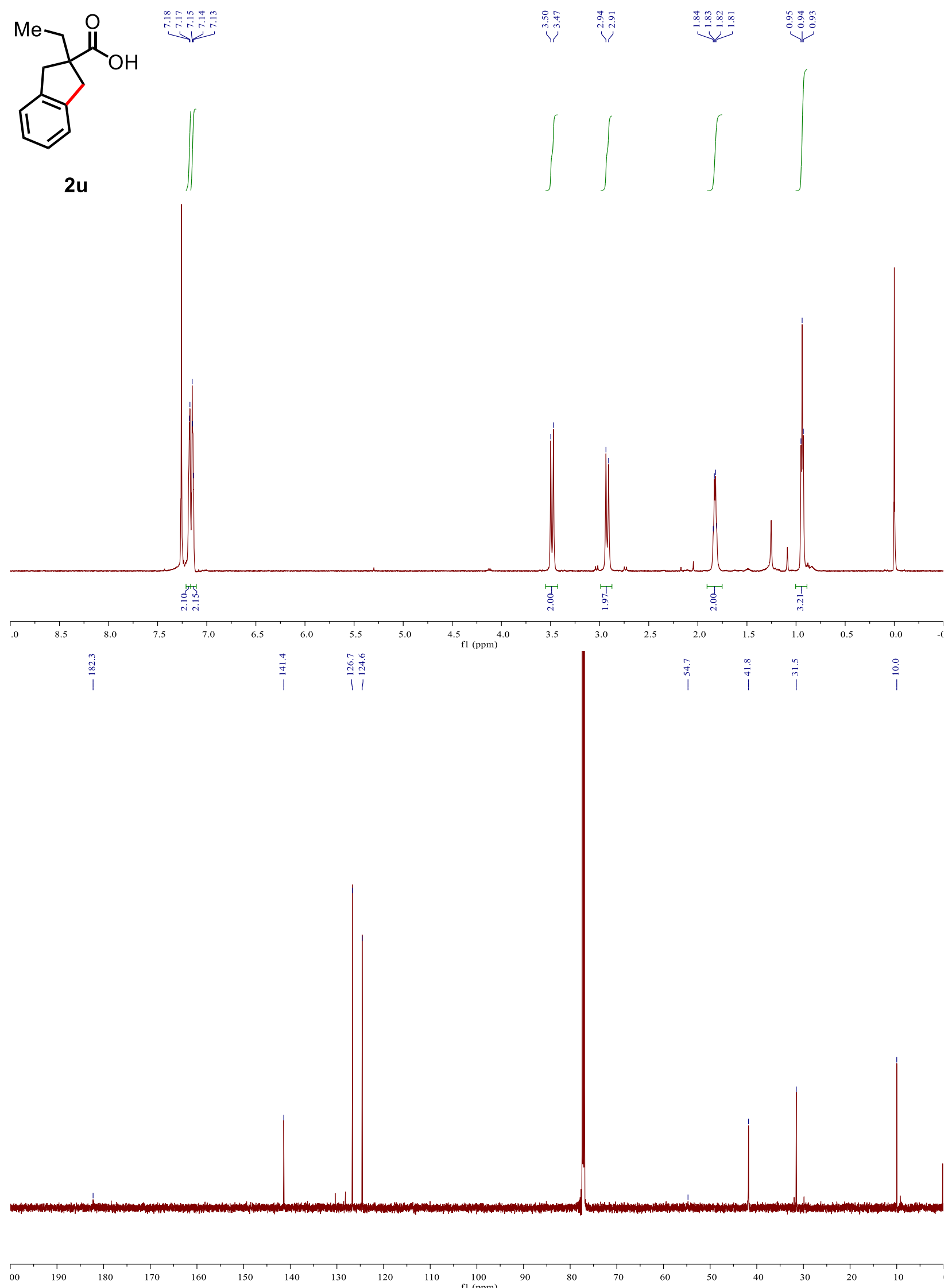


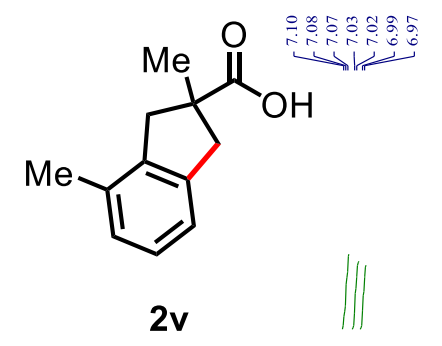

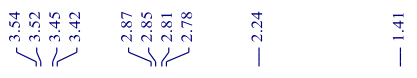
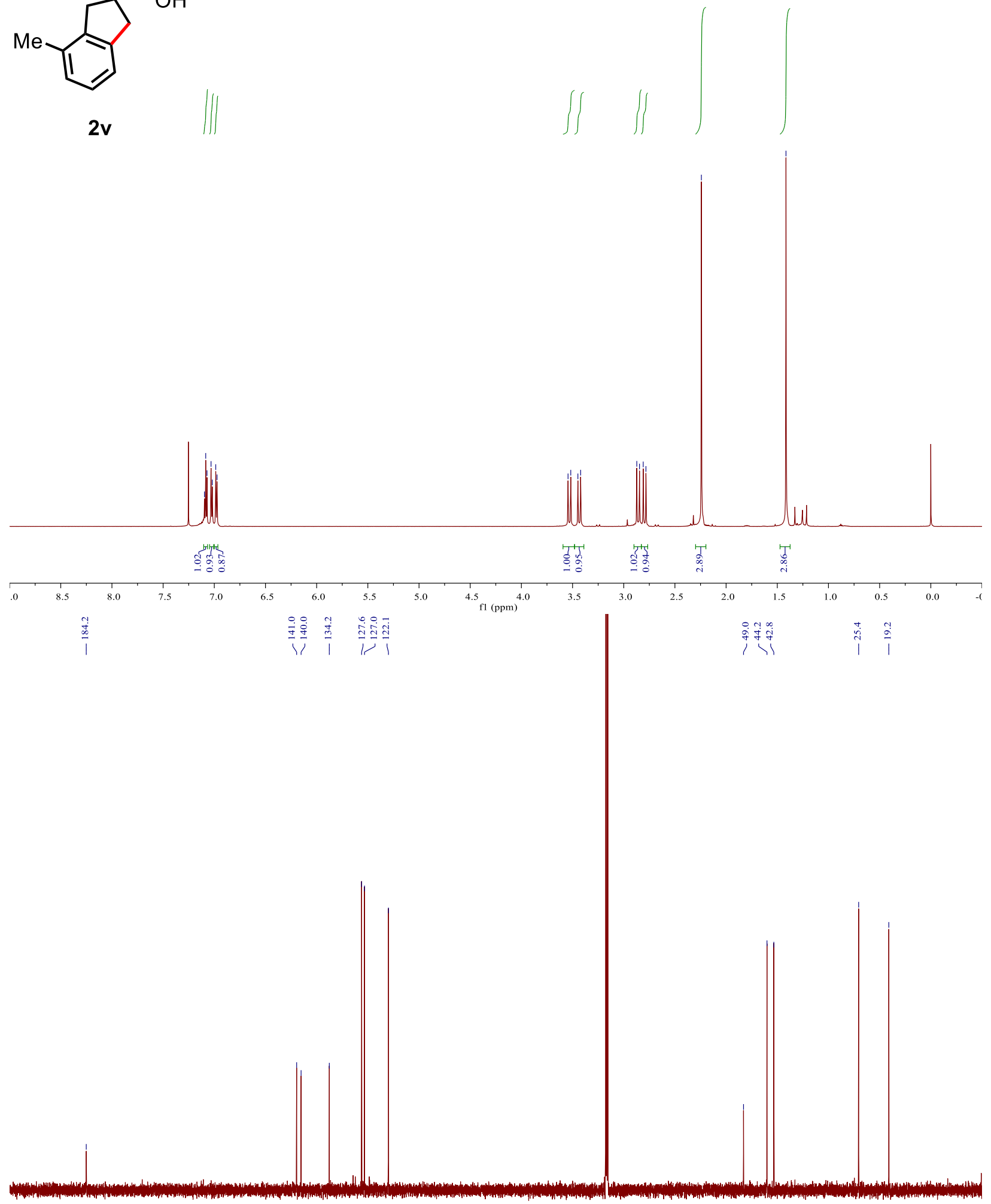

00

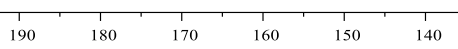

100
$\mathrm{fl}(\mathrm{ppm})$ 

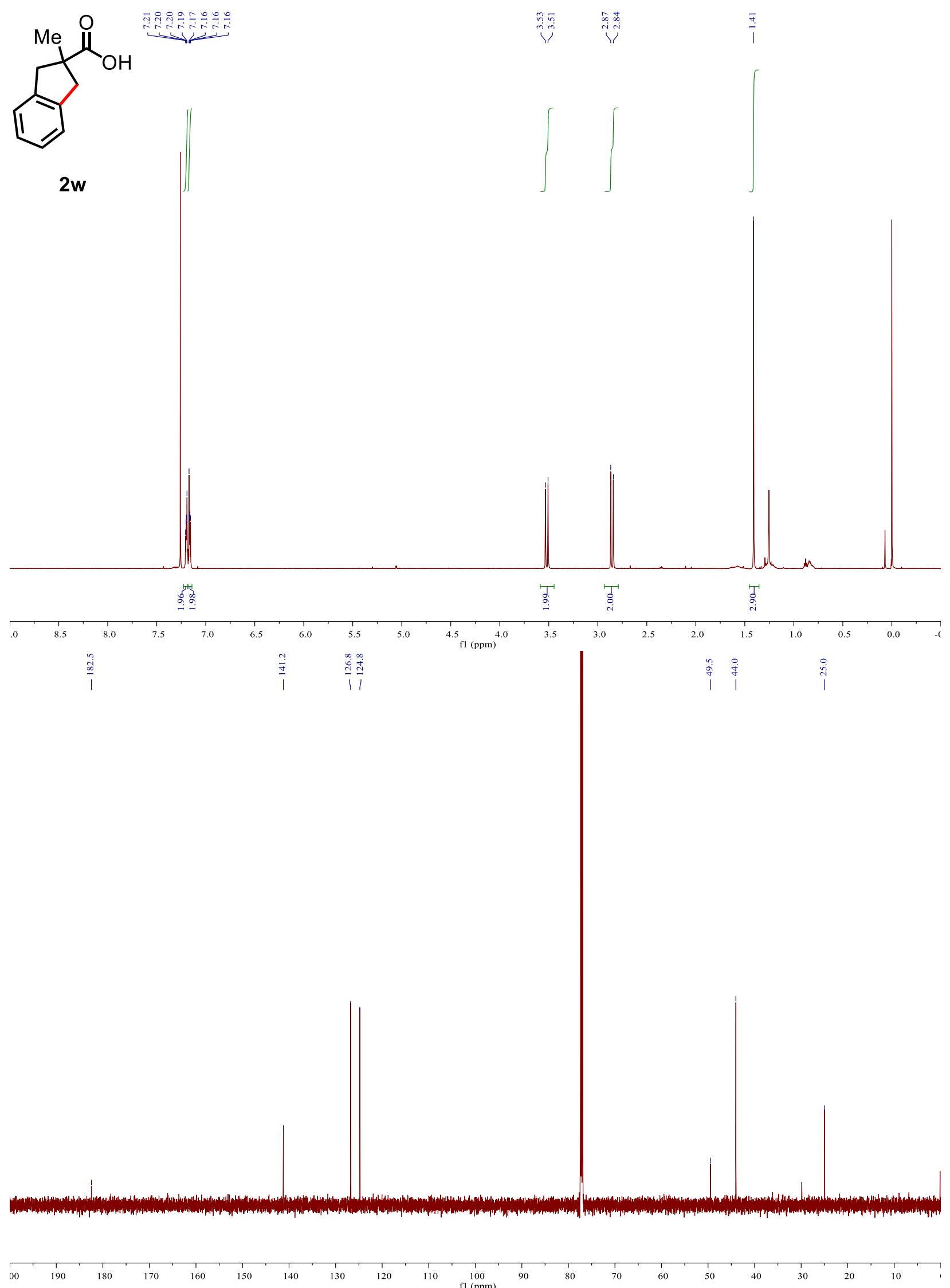

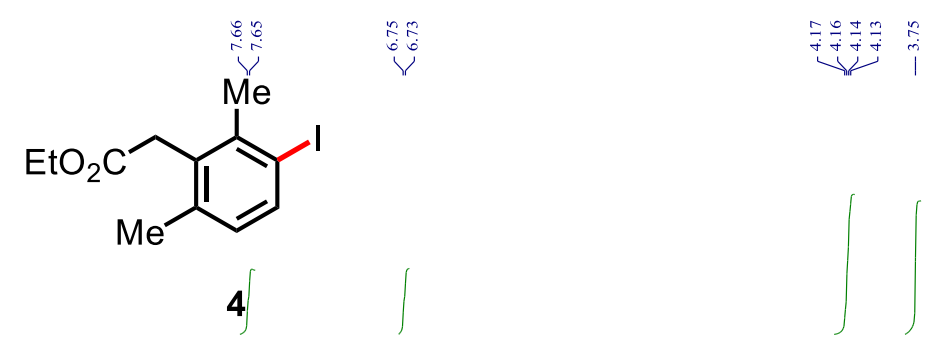

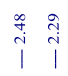

لِ
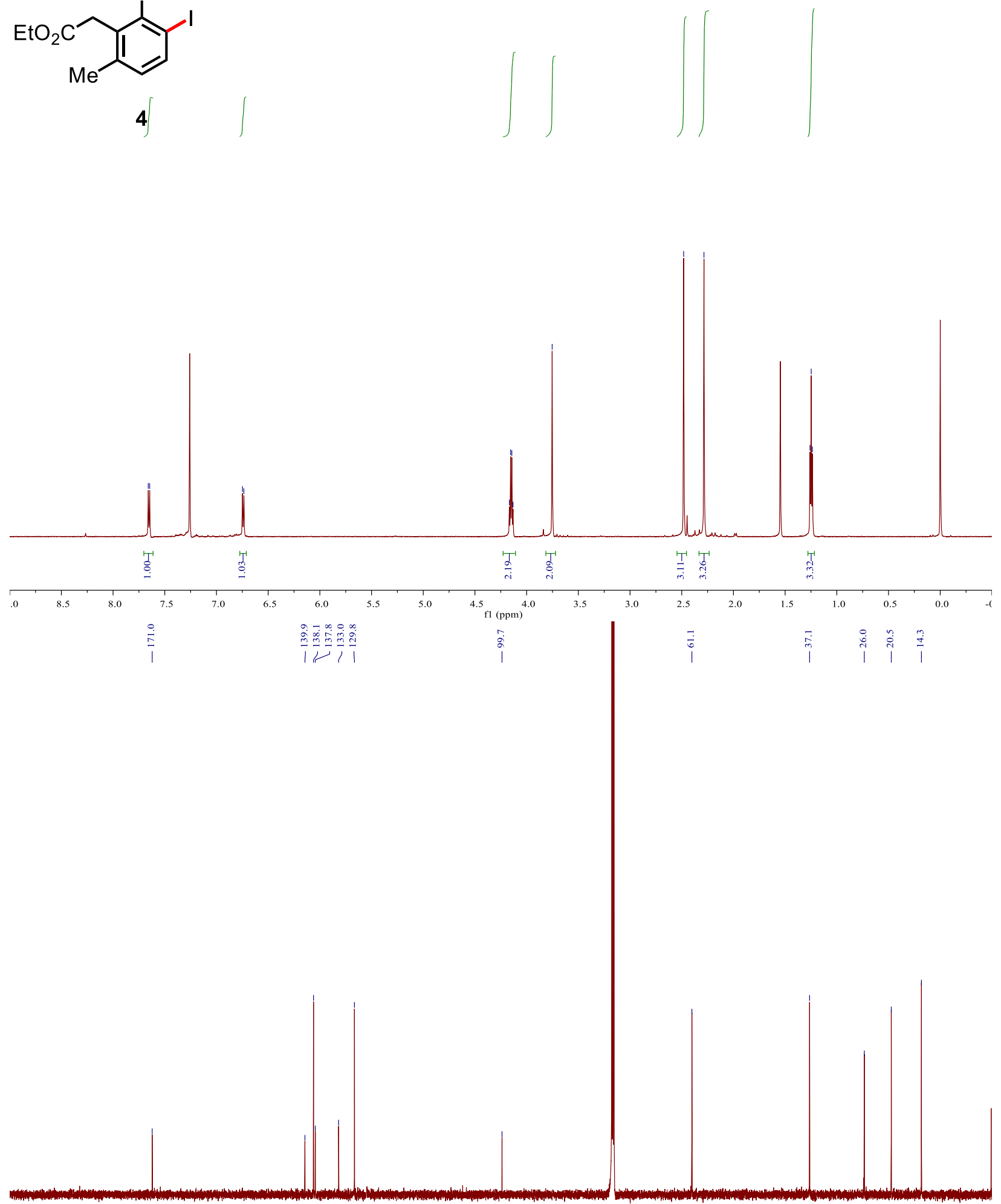

00

100
f1 $(\mathrm{ppm})$ 

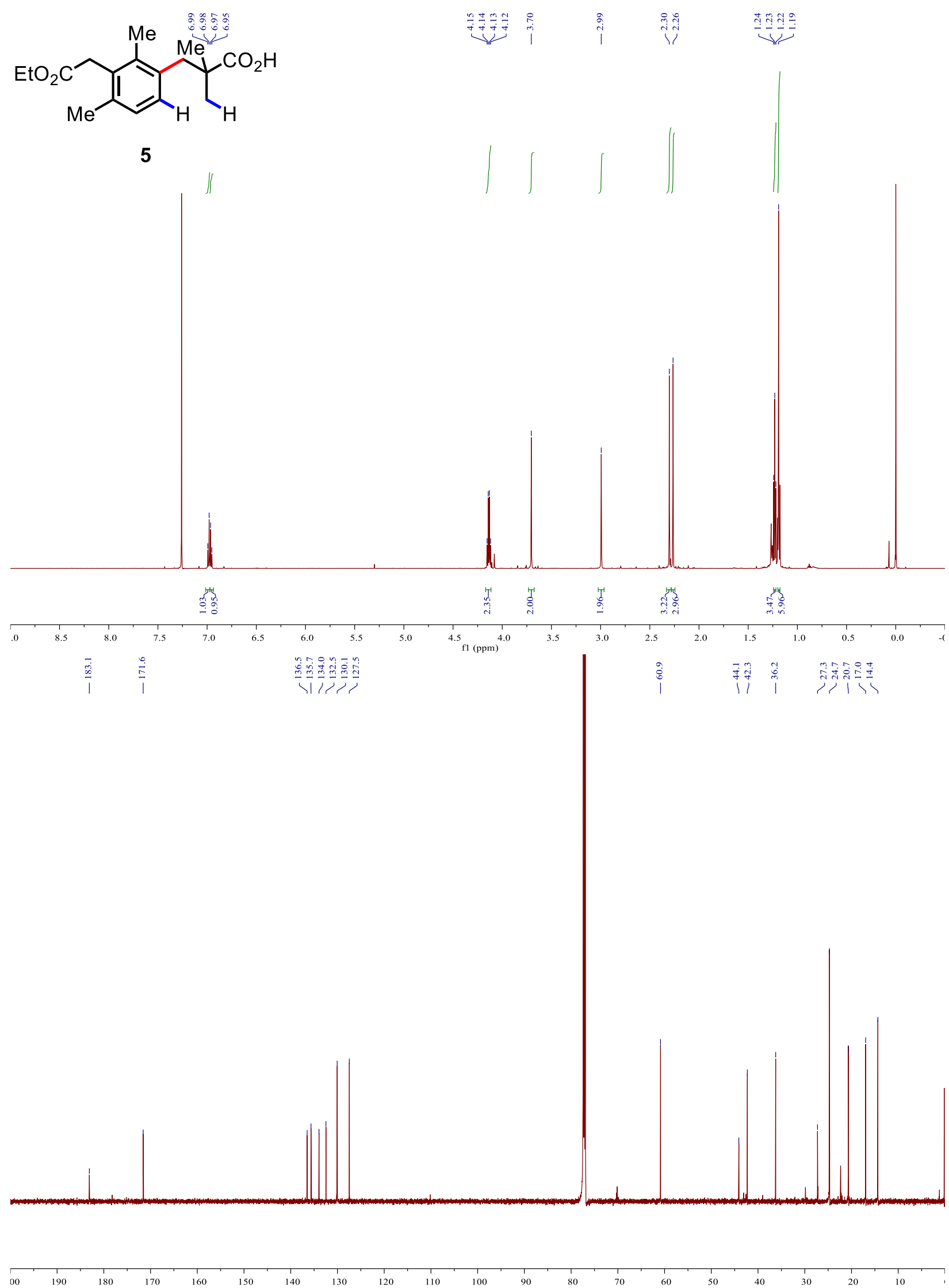

1 (ppm) 
<smiles>CCOC(=O)Cc1c(C)cc2c(c1C)C1C(C)C2C(C)(C(=O)O)C1C</smiles>

6

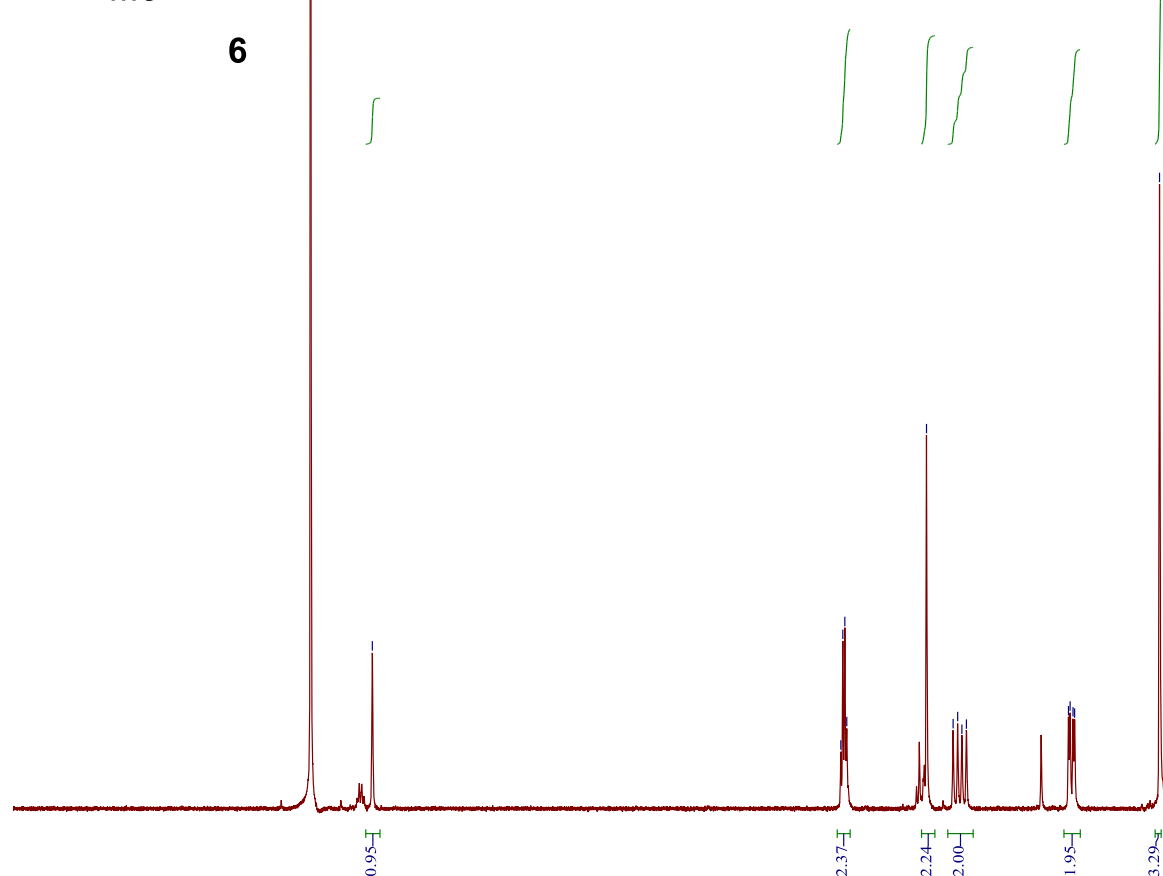

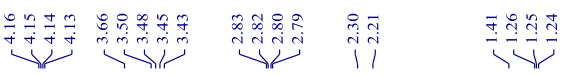

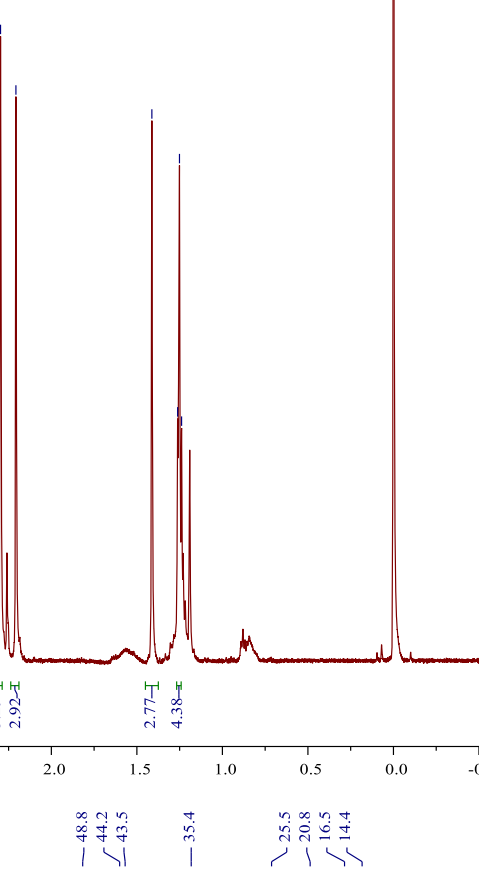

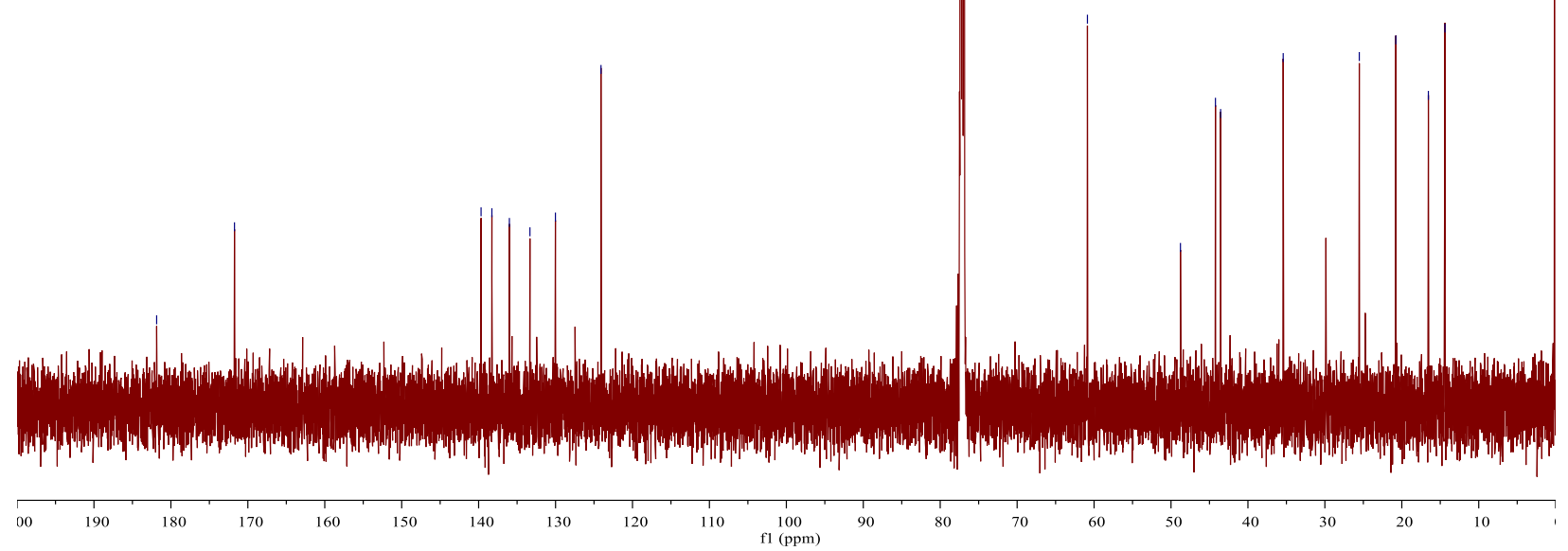


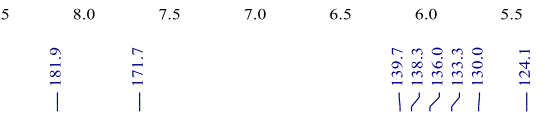




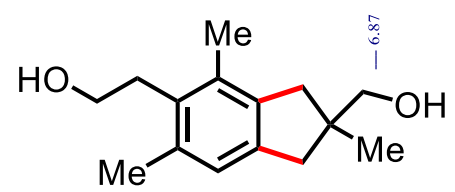

( \pm )-russujaponol F

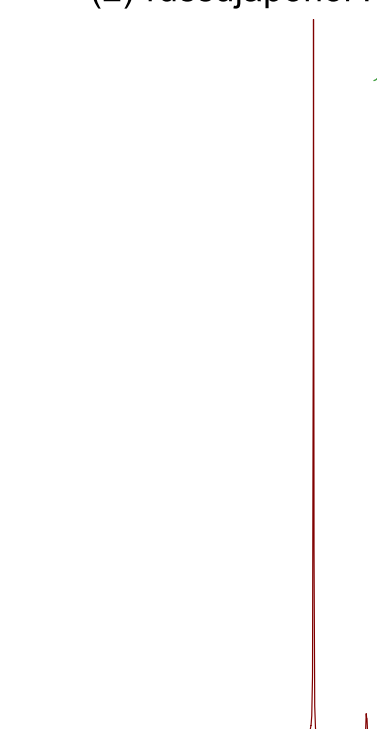

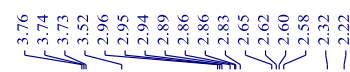

$\stackrel{\infty}{i}$
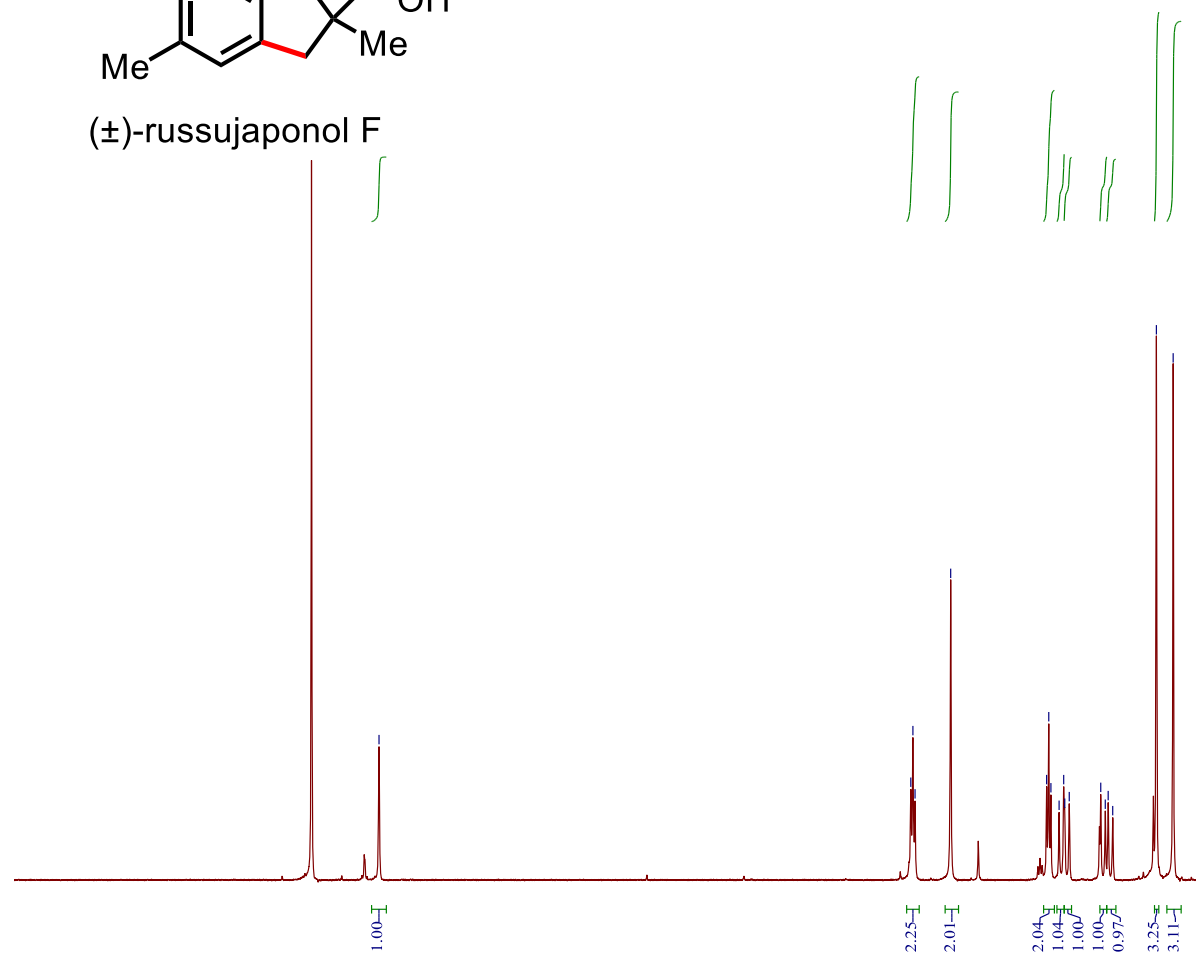

\section{管}

装等

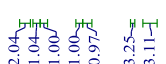

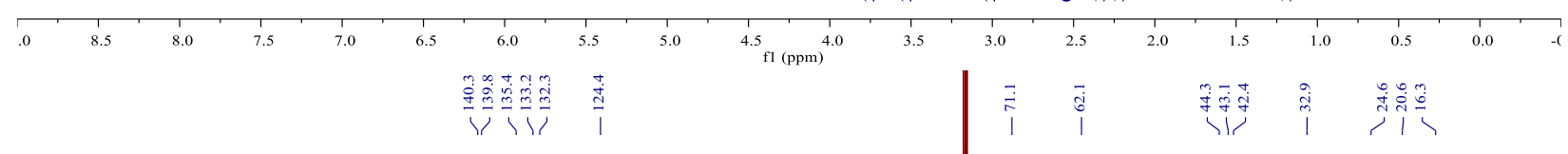

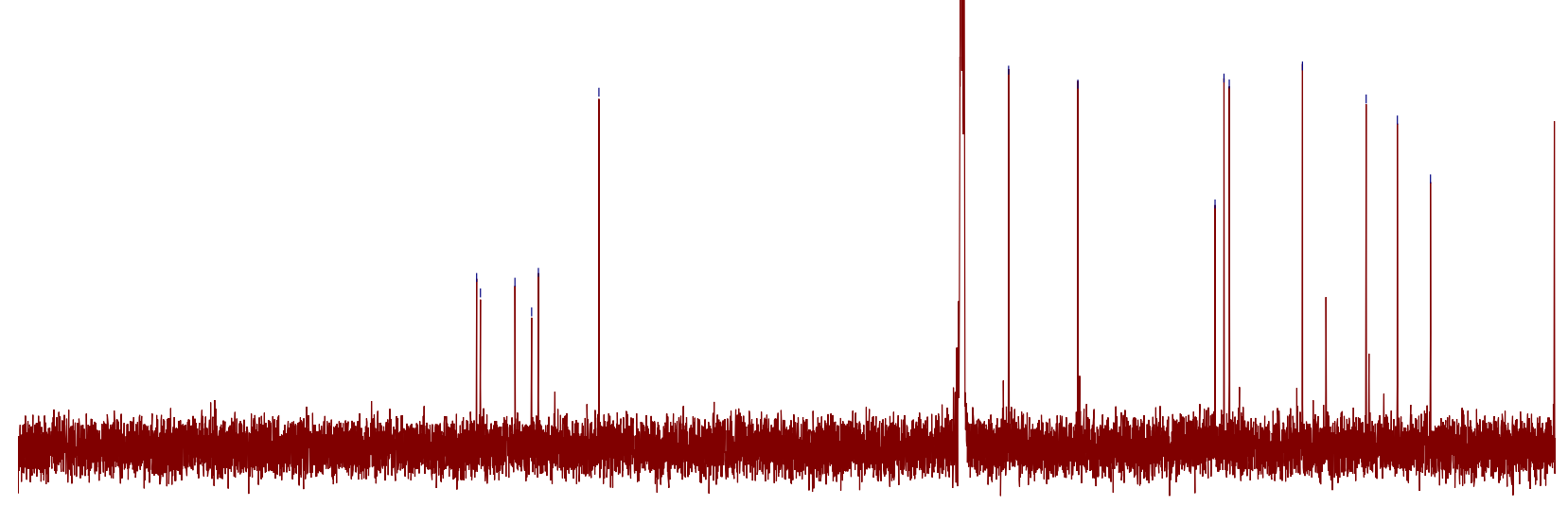

00

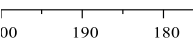

140

130

100
f1 $(\mathrm{ppm})$ 